\title{
Zur Theorie der elektrischen Ladung und der Koagulation der Kolloide
}

\section{Doctoral Thesis}

Author(s):

Müller, Hans

Publication date:

1928

Permanent link:

https://doi.org/10.3929/ethz-a-000098909

Rights / license:

In Copyright - Non-Commercial Use Permitted 
Diss. ET H $: 483 B$

\section{Zur Theorie der elektrischen Ladung und der Koagulation der Kolloide}

Von der

Eidgenössischen Technischen Hochschule in Zürich

zur Erlangung der

Würde eines Doktors der Naturwissenschaften

genehmigte

Nr. 483

Promotionsarbeit

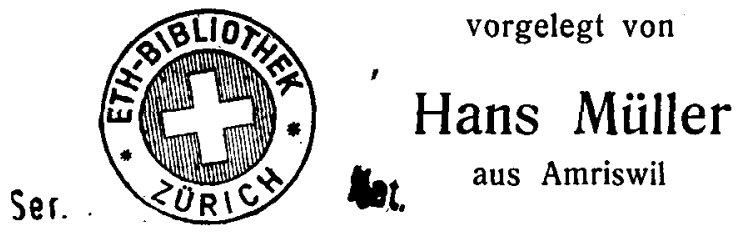

Referent: Herr Prof. Dr. P. Scherrer

Korreferent: Herr Prof. Dr. G. Wiegner

DRESDEN und LEIPZIG 1928

Theodor Steinkopff, Verlagsbuchhandlung

ETHICS ETH-BIB

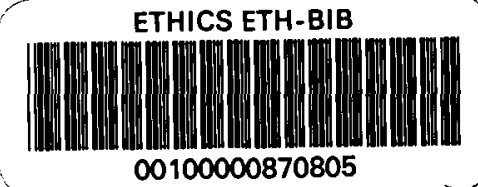


Die Arbeit erscheint gleichzeitig in den ,Kolloidchemischen Beiheften“ (Ergänzungshefte zur "Kolloid-Zeitschrift"), Monographien für reine und angewandte Kolloidchemie, Band XXVI, Heft 8-9. Herausgegeben von Prof. Dr. Wo. Ostwald. (Verlag von Theodor Steinkopff, Dresden und Leipzig.)

Druck von B. G. Teubner, Dresden. 


\section{Einleitung und Zusammenfassung.}

Das Problem der Stabilität eines Kolloides steht bekanntlich in: engem Zusammenhang mit den elektrischen Eigenschaften der Micellen. Die Erscheinung der Kataphorese einerseits und die chemisch analytischen Untersuchungen der Sole anderseits haben dazu geführt, um die Teilchen eine elektrische Doppelschicht anzunehmen, die einen Potentialsprung zwischen der Flüssigkeit und der Micelle bedingt. (Doppelschichttheorie von Helmholtz.) Dieser Potentialsprung ist die einzige Ursache der Existenzmöglichkeit des kolloidalen Zustandes. Infolge dieser Potentialdifferenz, die für gleiche Teilchen das gleiche Vorzeichen hat, stoßen sich die Micellen ab. Wird dagegen das Potential erniedrigt, was z. B. durch einen Elektrolytzusatz geschieht, so ist es möglich, daß für einige Micellen die Energie der Temperaturbewegung genügt, um die abstoßenden Kräfte zu überwinden. Sie stoßen dann zusammen und bleiben aneinander haften. Man nennt diese Erscheinung Koagulation. Durch weitere Erniedrigung des Potentials wird die Koagulationsgeschwindigkeit vergrößert, bis endlich die Potentialdifferenz so gering wird, daß jeder Zusammenstoß von zwei Teilchen zur Vereinigung führt. Man spricht dann von ,rascher Koagulation", im Gegensatz zur „langsamen Koagulation“, wenn nur ein Teil der Stöße-zur Komplexbildung führt.

v. Smoluchowski hat eine sehr schöne Theorie der raschen Koagulation gegeben für den Fall eines monodispersen Kolloides. Der erste Teil dieser Arbeit gibt eine Verallgemeinerung dieser Theorie für polydisperse Systeme.

Im zweiten Teil soll das Problem des elektrokinetischen Potentials und seine Abhängigkeit von der Elektrolytkonzentration näher studiert werden. Im Gegensatz zu der bis heute meist vertretenen Auffassung, die die Änderung des Potentials auf Änderung der adsorbierten Ladung allein zurückführt, wird gezeigt, daß die Theorie der diffusen Doppelschicht eine große Anzahl der experimentellen Tatsachen allein durch 
Änderung der Doppelschichtdicke erklären kann. Es wird eine Methode entwickelt, die erlaubt, den Einfluß der Adsorptionsänderung und denjenigen der Doppelschichtänderung voneinander zu trennen, und auf diese Weise aus kataphoretischen Messungen auf die Adsorptionsverhältnisse $\mathrm{zu}$ schließen. 


\title{
Erster Teil. \\ Die Theorie der Koagulation polydisperser Systeme.
}

\author{
(Mit 4 Figuren.)
}

Durch die grundlegende Theorie von v. Smoluchowski'), die durch die experimentellen Arbeiten von $Z$ sigmondi"), Westgren und Reitstötter ${ }^{3}$ ) so glänzend bestätigt wurde, beherrscht man den Ausflockungsprozeß bei der raschen Koagulation vollkommen. Diese Theorie geht davon aus, da $z$ wei kugelförmige Teilchen immer dann aneinander haften bleiben, wenn ihre Mittelpunkte infolge der Brownschen Bewegung in einen Abstand A kommen. Die Experimente haben gezeigt, daß dieser Abstand gerade gleich dem Teilchendurchmesser $2 \varrho$ ist, d. h. daß für Koagulation Berührung der Micellen notwendig ist.

Alle bisherigen Arbeiten setzen aber voraus, daß das Ausgangskolloid nur Teilchen gleicher Größe besitze. Diese Bedingung ist jedoch experimentell nur annäherungsweise erfüllbar und trifft bei den Kolloiden, mit denen es der Chemiker praktisch zu tun hat, überhaupt nie zu. Es ist daher für den Kolloidchemiker von speziellem Interesse, den Koagulationsverlauf in einem Gemisch von Micellen verschiedener Größe zu kennen.

Schon bevor v. Smoluchowski seine Theorie aufgestellt hatte, haben $1911 \mathrm{Wiegner}^{4}$ ) und Galecki ${ }^{5}$ ) aus Messungen an Milch und

1) v. Smoluchowski, Physik. Zeitschr. 17, 557/583 (1916); Zeitschr. f. phys. Chem. 92, 129 (1917); siehe auch Freundlich, Kapillarchemie.

2) Zsigmondi, Zeitschr. f. phys. Chem. 92, 545 (1917).

3) Westgren u. Reitstötter, Zeitschr. f. phys. Chem. 92, 750 (1917).

4) Wiegner, Koll.Zeitschr. 8, 227 (1911).

5) Galecki, Zeitschr. f. anorg. Chem. 74, 174 (1912). 
Goldsolen geschlossen, daß in einem polydispersen Kolloid die großen Micellen als Koagulationskeime für die kleinen Teilchen wirken. Sie konstatierten nämlich, daß bei der Flockung solcher Sole die Amikronen sehr rasch verschwinden, ohne daß gleichzeitig die Zahl der Submikronen wesentlich vergrößert wird. Sie zogen daraus die Folgerung, daß sich die Amikronen nicht, wie in einem monodispersen Kolloid, zu Submikronen zusammengeballt haben, sondern daß sie sich vielmehr an die schon in der Lösung befindlichen Submikronen angelagert haben müssen.

Es ist der Zweck der vorliegenden Arbeit, zu zeigen, daß dieser Effekt nicht im Widerspruch steht mit der Theorie von v. Smoluchowski, sondern daß er zwanglos aus den Ansätzen dieser Theorie abgeleitet werden kann.

Zur Behandlung dieses Problems, dessen Anregung ich Herrn Prof. Dr. G. Wi egner verdanke, wollen wir die Annahmen, die v. Smoluchowski zu seiner Theorie der raschen Koagulation eines monodispersen Sols geführt haben, zugrunde legen. Es wird also angenommen, daß ein Kolloidteilchen sich dann mit einem andern vereinigt, wenn es allein infolge seiner Brownschen Bewegung auf dieses stößt. Es wird also vorausgesetzt, daß keinerlei elektrische Kräfte zwischen den Micellen diesen Zusammenstoß begünstigen oder behindern. Wir können somit für den Zusammenstoß zweier Einzelteilchen genau den gleichen Ansatz machen wie v. Smoluchowski ${ }^{1}$ ) und verweisen für die Ableitung desselben wie auch für die Begründung der Rechnungsmethode auf dessen Originalarbeiten.

Wir betrachten ein Kolloid mit verschiedenen Teilchensorten $1,2,3,4, \ldots \mathrm{i}, \ldots$, die alle als kugelförmig vorausgesetzt werden und die sich nur durch die Größe der Teilchen unterscheiden. Die Anzahl der Teilchen der Sorte i auf $1 \mathrm{~cm}^{3}$ sei $\boldsymbol{\nu}_{\mathbf{i}^{*}}$ Greifen wir nun irgendein Teilchen einer beliebigen Sorte $k$ heraus, so berechnet sich nach Smoluchowskis Überlegungen die Anzahl der Teilchen i, die in der Zeit dt an dieses $\mathrm{k}$-Teilchen koagulieren, zu

$$
\delta n_{i k}=2 W_{i k} \nu_{i} d t .
$$

Die Größe $W_{\mathbf{i k}}$ ist ein Maß für die Wahrscheinlichkeit eines Zusammenstoßes eines $\mathrm{i}$-Teilchens und eines $\mathrm{k}$-Teilchens. Infolge der vorausgesetzten Kugelgestalt der Micellen erhält man:

$$
\mathrm{W}_{\mathrm{ik}}=2 \pi \mathrm{A}_{\mathrm{ik}} \mathrm{D}_{\mathrm{ik}} \text {. }
$$

Dabei ist $A_{i k}$ der Wirkungsradius für die Koagulation von i-Teilchen mit k-Teilchen, d. h. $A_{i k}$ ist der Abstand, in den der Mittelpunkt eines

1) Loc. cit. S. 5 . 
i-Teilchens vom Mittelpunkt eines $k$-Teilchens mindestens gelangen muß, um an diesem haften zu bleiben. $A_{i k}$ ist somit von der Größenordnung der Teilchenradien.

$D_{i k}$ bedeutet die Diffusionskonstante der $\mathrm{i}$-Teilchen reiativ zu einem k-Teilchen. Sind $D_{i}$ und $D_{k}$ die Diffusionskonstanten der beiden Teilchensorten, so gilt nach Smoluchowski allgemein

$$
D_{i k}=D_{i}+D_{k} \text {. }
$$

Die Berechnung von $\mathrm{W}_{\mathrm{ik}}$ setzt voraus, daß

$$
4 \pi \mathrm{A}_{\mathrm{ik}}^{3} \boldsymbol{v}_{\mathrm{i}}<<1,
$$

eine Bedingung, die in der Regel wegen der geringen Konzentration der Kolloide für alle Teilchensorten erfüllt ist.

Betrachten wir speziell die Anlagerung von i-Teilchen an ein Teilchen derselben Sorte $\mathrm{i}$, so wird

$$
\mathrm{W}_{\mathrm{ii}}=2 \pi \mathrm{A}_{\mathrm{ii}} 2 \mathrm{D}_{\mathrm{i}} \text {. }
$$

Bezeichnet man mit $\underline{o}_{\mathrm{i}}$ den Radius der $\mathrm{i}$-Teilchen, so ist bekanntlich nach Einstein

$$
\mathrm{D}_{\mathrm{i}}=\frac{\mathrm{k} \Theta}{6 \pi \eta \rho_{\mathrm{i}}}
$$

worin

$$
\begin{aligned}
& \mathrm{k}=\text { Boltzmannsche Konstante }=\frac{\text { Gaskonstante } \mathrm{R}}{\text { Avogadro-Zahl }} \\
& \Theta=\text { absolute Temperatur, } \\
& \eta=\text { Viskosität des Lösungsmittels (Wasser). }
\end{aligned}
$$

Da aber anderseits $A_{i i}$ proportional mit $\underline{o}_{\mathrm{i}}$ wachsen wird, so ergibt sich

$$
\mathrm{W}_{\mathrm{ii}}=\frac{2}{3} \frac{\mathrm{k} \Theta}{\eta}\left(\frac{\mathrm{A}_{\mathrm{ii}}}{e_{\mathrm{i}}}\right)=\mathrm{W}
$$

als unabhängig von der Teilchengröße.

Diese Unabhängigkeit des $\mathrm{W}$ vom Teilchenradius erklärt die Tatsache, daß bei der Ausflockung eines monodispersen Kolloides die Koagulationszeit unabhängig ist vom Dispersitätsgrad und allein von der Konzentration abhängt

Wenn die Teilchen $i$ und $k$ nicht wesentlich verschieden groß sind, so wird $\mathrm{W}_{\mathrm{ik}}$ nicht stark von $\mathrm{W}$ abweichen. Tatsächlich rechnet auch v. Smoluchowski für alle Zusammenstöße von beliebigen Mehrfach. teilchen mit einem konstanten W. Das ist aber nur erlaubt, solange nicht wesentlich verschiedene Teilchengrößen im Kolloid auftreten, also auch für ein monodisperses System nur am Anfang des Prozesses. Sobald man aber schon im Ausgangskolloid große und kleine Micellen 
nebeneinander hat, darf man $W_{i k}$ nicht mehr für alle Stöße gleich annehmen.

Sind die Teilchen $\mathrm{i}$ und $\mathrm{k}$ stark verschieden, d. h. ist $\varrho_{\mathbf{i}}>\varrho_{\mathrm{k}}$, so hat man also nach den Formeln (2), (3) und (5) genauer zu setzen:

$$
\begin{gathered}
\mathrm{W}_{\mathrm{ik}}=\frac{2}{3} \frac{\mathrm{k} \Theta}{\eta} \frac{\mathrm{A}_{\mathrm{ik}}}{2}\left(\frac{1}{\varrho_{\mathrm{i}}}+\frac{1}{\varrho_{\mathrm{k}}}\right)=\gamma_{\mathrm{ik}} \mathrm{W}, \\
\gamma_{\mathrm{ik}}=\left(\frac{1}{\varrho_{\mathrm{i}}}+\frac{1}{\varrho_{\mathrm{k}}}\right) \frac{\mathrm{A}_{\mathrm{ik}}}{2}\left(\frac{\rho_{\mathrm{i}}}{\mathrm{A}_{\mathrm{ii}}}\right) .
\end{gathered}
$$

Dieser Faktor $\gamma_{\mathrm{ik}}$ charakterisiert also die Verschiedenheit der Teilchengrößen. Apriori läßt sich über $\gamma_{i k}$ nichts aussagen, da wir den Wirkungsradius $A_{i k}$ für die Koagulation verschieden großer Teilchen nicht kennen, solange man nicht weiß, welche Kräfte man für das Zusammenballen der Teilchen verantwortlich zu machen hat. Die Versuche von Westgren und Reitstötter $r^{1}$ ) zeigten aber, daß im Falle eines monodispersen Systems $A_{i \mathrm{i}}=2 \varrho_{\mathrm{i}}$ zu setzen ist. Dadurch wird die Auffassung nahegelegt, da $B$ für die Koagulation der Teilchen Berührung derselben notwendig ist. Wir wollen daher diese Bedingung auch für das $\mathrm{Zu}$ sammenballen verschieden großer Teilchen einführen. Die Berechtigung dieser Hypothese kann natürlich nur durch den Vergleich ihrer Folgerungen mit der Erfahrung geprüft werden. Wir setzen also

und

$$
A_{i \mathbf{i}}=2 \varrho_{\mathrm{i}}
$$

$$
A_{i k}=\varrho_{i}+\varrho_{k} \text {, }
$$

wodurch wir für $\gamma_{\mathrm{ik}}$ folgenden Ausdruck erhalten:

$$
\gamma_{\mathrm{ik}}=\frac{\left(\varrho_{\mathrm{i}}+\varrho_{\mathrm{k}}\right)^{2}}{4 \varrho_{\mathrm{i}} \varrho_{\mathrm{k}}}=\frac{\left(1+\frac{\rho_{\mathrm{i}}}{\varrho_{\mathrm{k}}}\right)^{2}}{4 \frac{\rho_{\mathrm{i}}}{\varrho_{\mathrm{k}}}} .
$$

Dieser Faktor $\dot{\gamma}_{\mathrm{ik}}$. der die Wahrscheinlichkeit des Zusammenstoßes zweier ungleich großer Teilchen charakterisiert, wird also nur cine Funktion des Verhältnisses $\frac{\varrho_{i}}{\varrho_{k}}$ der Teilchenradien. Diese Funktion muß natürlich, wie die erste Form zeigt, vollkommen symmetrisch in $\varrho_{\mathbf{i}}$ und $\varrho_{k}$ sein. Sie hat für $\frac{\varrho_{i}}{\varrho_{k}}=1$. das einzige Minimum $\gamma_{i k}=1$. Da somit immer $\gamma_{i k}>1$ (ausgenommen wenn die Teilchen gleich sind), so folgt daraus, daß der Zusammenstoß von zwei ungleichen Teilchen immer

1) Loc. cit. S. 5 . 
wahrscheinlicher ist als der Zusammenstoß von zwei gleich großen. Diese Überlegung erklärt uns bereits, daß der von Wiegner gefundene Effekt existieren muß.

Um den Einfluß dieser Tatsache zu übersehen, betrachten wir jetzt einfacher ein Kolloid, in dem nur zwei Teilchensorten auftreten. Es seien darin auf $1 \mathrm{cbm} n_{0}$,kleine Teilchen" vom Radius $r$ und $N_{0}$ „große Teilchen" vom Radius R. Wir setzen ferner

$$
\frac{N_{0}}{n_{0}}=V_{n} \text { und } \frac{R}{r}=V_{R} \text {. }
$$

Dann wird der Wahrscheinlichkeitsfaktor für den Zusammenstoß dieser zwei Teilchensorten

$$
\gamma_{\mathrm{Rr}}=\frac{\left(1+\mathrm{V}_{\mathrm{R}}\right)^{2}}{4 \mathrm{~V}_{\mathrm{R}}}=\gamma>1 \text {. }
$$

Entsprechend dem Verfahren von v. Smoluchowski benutzen wir nun für alle Zusammenstöße von kleinen Teilchen oder Komplexen derselben untereinander den konstanten Wahrscheinlichkeitsfaktor W und ebenso für alle Zusammenstöße von großen Teilchen oder beliebigen Komplexen derselben untereinảnder. Jedoch für alle Zusammenstöße von kleinen Teilchen oder Komplexen von nur solchen mit großen Teilchen oder Komplexen derselben setzen wir

$$
\mathrm{W}_{\mathrm{Rr}}=\gamma_{\mathrm{Rr}} \mathrm{W},
$$

indem wir für alle diese letzteren Stöße $\gamma_{\mathbf{R}_{r}}=\gamma$ als konstant annehmen.

Dieses Verfahren setzt allerdings voraus, daß auch die Komplexteilchen noch als Kugeln betrachtet werden können und daß auch für die Komplexteilchen im Mittel das Radienverhältnis $\mathrm{V}_{\mathrm{R}}$ erhalten bleibt. Beide Annahmen sind zwar kaum streng richtig, aber die Rechnungsart ist wohl deshalb erlaubt, weil die Mehrfachteilchen am Anfang der Koagulation relativ selten sind.

Fassen wir endlich die großen Micellen als Komplexe von $\mathrm{k}$ kleinen Teilchen auf, so können wir für $\boldsymbol{\nu}_{\mathrm{i}}$, das ist die Zahl derjenigen Teilchen, die aus i kleinen Teilchen gebildet sind, die Differentialgleichung aufstellen, indem wir abzählen, wieviel solcher Teilchen in der Zeit dt gebildet werden und wieviel durch Zusammenstöße mit anderen Teilchen verschwinden. Wir erhalten so, durch die genau gleiche Überlegung wie v. Smoluchowski, folgendes System von unendlich vielen Differentialgleichungen: 
$\frac{1}{2} \frac{\mathrm{d} \nu_{1}}{\mathrm{dt}}=$

$$
\begin{array}{ll}
\frac{1}{2 \mathrm{~W}} \frac{\mathrm{d} \nu_{1}}{\mathrm{dt}}= & -\nu_{1} \sum_{\mathrm{i}}^{\mathrm{k}-1} \nu_{\mathrm{i}}-\gamma \nu_{1} \sum_{\mathrm{k}}^{\infty} \nu_{\mathrm{i}} \\
\frac{1}{2 \mathrm{~W}} \frac{\mathrm{d} \nu_{2}}{\mathrm{dt}}=\frac{1}{2} \nu_{1}^{2} & -\nu_{2} \sum_{1}^{\mathrm{k}-1} \nu_{\mathrm{i}}-\gamma \nu_{2} \sum_{\mathrm{k}}^{\infty} \nu_{\mathrm{i}} \\
\frac{1}{2 \mathrm{~W}} \frac{\mathrm{d} \nu_{3}}{\mathrm{dt}}=\frac{1}{2}\left(\nu_{1} \nu_{2}+\nu_{2} \nu_{1}\right)-\nu_{3} \sum_{1}^{k-1} \nu_{\mathrm{i}}-\gamma \nu_{3} \sum_{\mathrm{k}}^{\infty} \nu_{\mathrm{i}}
\end{array}
$$

$\frac{1}{2 \mathrm{~W}} \frac{\mathrm{d} \nu_{\mathrm{k}-1}}{\mathrm{dt}}=\frac{1}{2}\left(\nu_{1} v_{\mathrm{k}-2}+\boldsymbol{v}_{2} \nu_{\mathrm{k}-3}+\ldots \nu_{\mathrm{k}-2} \nu_{1}\right)$

$$
-\nu_{k-1} \sum_{1}^{k-1} \nu_{i}-\gamma \nu_{k-1} \sum_{k}^{\infty} \nu_{i}
$$

$\frac{1}{2 \mathrm{~W}} \frac{\mathrm{d} \nu_{\mathrm{k}}}{\mathrm{dt}}=\frac{1}{2}\left(\nu_{1} \nu_{\mathrm{k}-1}+\ldots \nu_{\mathrm{k}-1} \nu_{1}\right)-\gamma \nu_{\mathrm{k}} \sum_{1}^{\mathrm{k}-1} \nu_{\mathrm{i}}-\nu_{\mathrm{k}} \sum_{\mathrm{k}}^{\infty} \nu_{\mathrm{i}}$ $\frac{1}{2 \mathrm{~W}} \frac{\mathrm{d} v_{k+1}}{\mathrm{dt}}=\frac{1}{2}\left(\nu_{2} \nu_{k-1}+\ldots \nu_{k-1} \nu_{2}\right)+\gamma \nu_{1} \nu_{k}$

$$
-\gamma \nu_{k+1} \sum_{1}^{k-1} v_{i}-\nu_{k+1} \sum_{k}^{\infty} \nu_{i}
$$

$\frac{1}{2 \mathrm{~W}} \frac{\mathrm{d} \nu_{\mathrm{k}+2}}{\mathrm{dt}}=\frac{1}{2}\left(\nu_{3} \nu_{\mathrm{k}-1}+\ldots \nu_{\mathrm{k}-1} \nu_{3}\right)+\gamma\left(\nu_{1} \nu_{\mathrm{k}+1}+v_{2} \nu_{\mathrm{k}}\right)$

$$
-\gamma \nu_{k+2} \sum_{1}^{k-1} v_{i}-\nu_{k+2} \sum_{k}^{\infty} \nu_{i}
$$

$\frac{1}{2 \mathrm{~W}} \frac{\mathrm{d} \nu_{2 \mathrm{k}-2}}{\mathrm{dt}}=\frac{1}{2} \nu_{\mathrm{k}-1}^{2}+\gamma\left(\nu_{1} \nu_{2 \mathrm{k}-3}+\ldots \nu_{\mathrm{k}-2} \nu_{\mathrm{k}}\right)$

$$
-\gamma \nu_{2 \mathrm{k}-2} \sum_{1}^{\mathrm{k}-1} \nu_{\mathrm{i}}-\nu_{2 \mathrm{k}-2} \sum_{\mathrm{k}}^{\infty} \nu_{\mathrm{i}}
$$

$\frac{1}{2 \mathrm{~W}} \frac{\mathrm{d} \nu_{2 \mathrm{k}-1}}{\mathrm{dt}}=+\gamma\left(\nu_{1} \nu_{2 \mathrm{k}-2}+\ldots \nu_{\mathrm{k}-1} \nu_{\mathrm{k}}\right)-\gamma \nu_{2 \mathrm{k}-1} \sum_{1}^{\mathrm{k}-1} \nu_{\mathrm{i}}-\nu_{2 \mathrm{k}-1} \sum_{\mathrm{k}}^{\infty} \nu$ $\frac{1}{2 \mathrm{~W}} \frac{\mathrm{d} \nu_{2 \mathrm{k}}}{\mathrm{dt}}=\frac{1}{2} \nu_{\mathrm{k}}^{2}+\gamma\left(\nu_{1} \nu_{2 \mathrm{k}-1}+\ldots \nu_{\mathrm{k}-1} \nu_{\mathrm{k}+1}\right)-\gamma \nu_{2 \mathrm{k}} \sum_{1}^{\mathrm{k}-1} \nu_{\mathrm{i}}-\nu_{2 \mathrm{k}} \sum_{\mathrm{k}}^{\infty} \nu_{\mathrm{i}}$ $\frac{1}{2 \mathrm{~W}} \frac{\mathrm{d} \nu_{2 \mathrm{k}+1}}{\mathrm{dt}}=\frac{1}{2}\left(\nu_{\mathrm{k}+1} \nu_{\mathrm{k}}+v_{\mathrm{k}} \nu_{\mathrm{k}+1}\right)+\gamma\left(\nu_{1} \nu_{2 \mathrm{k}}+\ldots \nu_{\mathrm{k}-1} \nu_{\mathrm{k}+2}\right)$

$$
-\gamma \nu_{2 k+1} \sum_{1}^{k-1} \nu_{i}-\nu_{2 k+1} \sum_{k}^{\infty} \nu_{i}
$$

$\frac{1}{2 \mathrm{~W}} \frac{\mathrm{d} \nu_{2 \mathrm{k}+2}}{\mathrm{dt}}=\frac{1}{2}\left(\nu_{\mathrm{k}+2} \nu_{\mathrm{k}}+\ldots \nu_{\mathrm{k}} \nu_{\mathrm{k}+2}\right)+\gamma\left(\nu_{1} \nu_{2 \mathrm{k}+1}+\ldots \nu_{\mathrm{k}-1} \nu_{\mathrm{k}+3}\right)$

$$
-\gamma \nu_{2 k+2} \sum_{1}^{k-1} \nu_{i}-\nu_{2 k+2} \sum_{k}^{\infty} \nu_{i}
$$


Den Chemiker interessiert aber nicht die Änderung der Anzahl der verschiedenen Komplexteilchen $i-$ die er in der Regel nicht voneinander unterscheiden kann -, sondern die Änderung der Gesamtzahl der großen und kleinen Teilchen. Dabei verstehen wir unter der Gesamtzahl der kleinen Teilchen die Zahl aller Komplexe, für die $\mathrm{i}<\mathrm{k}$ und analog als Gesamtzahl der großen Teilchen die Zahl aller Komplexe, für die $\mathrm{i} \geqq k$. Die ultramikroskopische Auszählung dieser Gesamtzahlen dürfte experimentell möglich sein, wenn die beiden Teilchensorten im Ausgangskolloid in ihrem Dispersitätsgrad stark verschieden sind.

Wir addieren daher alle Differentialgleichungen und erhalten

$$
\begin{aligned}
\frac{1}{2 \bar{W}} \frac{\mathrm{d}}{\mathrm{d} t}\left(\sum_{1}^{\mathrm{k}-1} \nu_{\mathrm{i}}\right. & \left.+\sum_{\mathrm{k}}^{\infty} \nu_{\mathrm{i}}\right)=-\left(\sum_{1}^{\mathrm{k}-1} \nu_{\mathrm{i}}\right)^{2}-\gamma \sum_{1}^{\mathrm{k}-1} \nu_{\mathrm{i}} \sum_{\mathrm{k}}^{\infty} \nu_{\mathrm{i}} \\
+\frac{1}{2}\left(\sum_{1}^{\mathrm{k}-1} \nu_{\mathrm{i}}\right)^{2} & +\frac{1}{2}\left(\sum_{\mathrm{k}}^{\infty} \nu_{\mathrm{i}}\right)^{2}-\gamma \sum_{\mathrm{i}}^{-1} \nu_{\mathrm{i}} \sum_{\mathrm{k}}^{\infty} \nu_{\mathrm{i}}-\left(\sum_{\mathrm{k}}^{\infty} \nu_{\mathrm{i}}\right)^{2} \\
& +\gamma \sum_{1}^{\mathrm{k}-1} \nu_{\mathrm{i}} \sum_{\mathrm{k}}^{\infty} \nu_{\mathrm{i}}
\end{aligned}
$$

and setzen darin zur Vereinfachung

$$
\begin{aligned}
& \sum_{i}^{\mathrm{k}-1} \nu_{\mathrm{i}}=\sum \boldsymbol{\nu} \text { Gesamtzahl der kleinen Teilchen } \\
& \sum_{\mathrm{k}}^{\infty} \nu_{\mathrm{i}}=\sum \mathrm{N} \text { Gesamtzahl der großen Teilchen. }
\end{aligned}
$$

Dann wird

$$
\frac{1}{2} \bar{W} \frac{d}{d t}\left(\sum v+\sum N\right)=-\frac{1}{2}\left(\sum \nu\right)^{2}-\gamma \sum v \sum N-\frac{1}{2}\left(\sum N\right)^{2}
$$

Wir machen nun die Annahme, daß die großen Teilchen bedeutend größer seien als dié kleinen. Diese. Bedingung muß für eine Prüfung der Theorie notwendig erfüllt sein, um eine möglichst große Abweichung von der v. Smoluchowskischen Theorie zu erhalten. In diesem Falle wird es einige Zeit dauern, bis die Anzahl der großen Teilchen merklich beeinflußt wird durch Bildung solcher aus lauter kleinen Teilchen. Für den Anfang der Koagulation dürfen wir also die Glieder, die diese Bildung berücksichtigen, vernachlässigen. Dann liefert aber die Summation der Differentialgleichungen von der $\mathrm{k}^{\text {ten }}$ an

$$
\frac{1}{2 \mathrm{~W}} \frac{\mathrm{d}}{\mathrm{d} t} \sum \mathrm{N}=-\frac{1}{2}\left(\sum N\right)^{2}
$$


woraus durch Integration

$$
\sum N=\frac{N_{0}}{1+\frac{t}{T}}
$$

wo $\mathrm{T}=\frac{1}{\mathrm{~N}_{0} \mathrm{~W}}$ die ausv.Smoluchowskis Theoriebekannte Koagulationszeit der großen Teilchen ist, das ist die Zeit, in der die Gesamtzahl der großen Teilchen, wenn sie allein im Kolloid wären, um die Hälfte abnimmt. Die Formeln (12) und (13) sind vollkommen identisch mit den Koagulationsformeln für ein monodisperses System. Das heißt nichts anderes, als daß mindestens im Anfang die großen Teilchen so koagulieren, als ob die kleinen Teilchen nicht da wären, ein Effekt, der nach unseren Voraussetzungen zu erwarten war, und auch durch die Wiegnerschen Messungen ${ }^{1}$ ) bestätigt wird. Eine Überschlagsrechnung zeigt, daß, wenn die ursprünglichen Anzahlen $N_{0}$ und $n_{0}$ gleich sind, und wenn jedes große Teilchen das Volumen von 100 kleinen ausmacht, Formel (13) mindestens bis zur Zeit $t=20 \mathrm{~T}$ auf mehr als 1 Proz. genau sein muß.

Da wir also in diesem Zeitintervall die Änderung von $\sum N$ kennen, so können wir aus (11') die Änderung der Gesamtzahl der kleinen Teilchen bestimmen. Wir erhalten somit, indem wir (13) in (11') einsetzen, für $\Sigma \nu$ folgende Differentialgleichung, die mindestens für den Anfang der Koagulation ( $t$ klein) gilt:

$$
\begin{gathered}
\frac{1}{2 \mathrm{~W}} \frac{\mathrm{d}}{\mathrm{dt}}\left(\sum \nu\right)=-\frac{1}{2}\left(\sum \nu\right)^{2}-\gamma \sum \nu \cdot \frac{\mathrm{N}_{0}}{1+\frac{\mathrm{t}}{\mathrm{T}}} \\
-\frac{1}{2} \frac{\mathrm{N}_{0}^{2}}{\left(1+\frac{\mathrm{t}}{\mathrm{T}}\right)^{2}}-\frac{1}{2 \mathrm{~W}} \frac{\mathrm{d}}{\mathrm{dt}}\left(\frac{\mathrm{N}_{0}}{1+\frac{\mathrm{t}}{\mathrm{T}}}\right) .
\end{gathered}
$$

Die zwei letzten Glieder heben einander auf, so daß

$$
\frac{1}{2} \overline{\mathrm{W}} \frac{\mathrm{d}}{\mathrm{dt}}\left(\sum \nu\right)=-\frac{1}{2}\left(\sum \nu\right)^{2}-\gamma \sum \nu \cdot \frac{\mathrm{N}_{0}}{1+\frac{\mathrm{t}}{\mathrm{T}}} .
$$

Diese Differentialgleichung vom Bernoullischen Typus löst man mit einem Ansatz ${ }^{2}$ )

$$
\sum v=u \cdot v
$$

Führt man an Stelle von $\gamma$ ein:

$$
\lambda=2 \gamma-1 \text {. }
$$

1) Loc. cit. S. 5 .

2) Siehe Anhang zum 1. Teil. 
so erhält man als Endformel die Lösung:

$$
\sum \nu=\frac{N_{0}}{1+\frac{t}{T}} \cdot \frac{\lambda n_{0}}{\left(N_{0} \lambda+n_{0}\right)\left(1+\frac{t}{T}\right)^{\lambda}-n_{0}} .
$$

Diese Formel (16) zusammen mit Gleichung (13) bildet das Hauptresultat unserer Untersuchung. Während (13) aussagt, daß die großen Teilchen nach v. Smoluchowski koagulieren, gibt (16) einen bedeutend andern Abfall für die Gesamtzahl $\boldsymbol{\Sigma} \boldsymbol{\nu}$ der kleinen Teilchen. Nur in dem Grenzfalle, wenn die kleinen Teilchen allein in der Lösung sind, also im Falle $\mathrm{N}=0$, muß (16) in die Koagulationsgleichung des monodispersen Systems übergehen, was auch tatsächlich zutrifft. Unser Kolloid geht auch in ein monodisperses System über, wenn $V_{R}=1$. In der Tat zeigt dann eine einfache Rechnung, daß die Summe von (13) und (16) die nach v. Smoluchowski zu erwartende Koagulationsgleichung liefert. ${ }^{\text {) }}$

Vorläufig sind unsere Gleichungen nur für den Anfang der Koagulation begründet. Wir wollen aber zeigen, daß sowohl (13) wie (16) den gesamten zeitlichen Verlauf der Flockung mit sehr guter Annäherung darstellen. Um dies zu beweisen, müssen wir die physikalische Aussage der Gleichung (16) eingehender diskutieren.

Aus der Definition (15) von $\lambda$ folgt nach $\left(9^{\prime}\right)$ :

$$
\lambda=2 \gamma-1=\frac{\left(1+\mathrm{V}_{\mathrm{R}}\right)^{2}}{2}-1=\frac{1+\mathrm{V}_{\mathrm{R}}{ }^{2}}{2 \mathrm{~V}_{\mathrm{R}}}
$$

Da aber für die ganze Überlegung das Radienverhältnis $V$ als groß vorausgesetzt wurde und da anderseits das Experiment für diese Größe nur einen Näherungswert liefern kann, so dürfen wir als eine gute $\mathrm{Ab}$ schätzung setzen:

$$
\lambda^{\prime}=\frac{\mathrm{V}_{\mathrm{R}}}{2} \text {. }
$$

Führen wir noch das Verhältnis $V_{N}=\frac{N_{0}}{n_{9}}$ der anfänglichen Teilchenzahlen ein, so erhalten wir für (16) folgende For $m$, in der nur noch direkt bekannte Größen auftreten:

$$
\sum \nu=\frac{\mathrm{N}_{0}}{\left(1+\frac{\mathrm{t}}{\mathrm{T}}\right)} \cdot \frac{\frac{\mathrm{V}_{\mathrm{R}}}{2}}{\left(\frac{\mathrm{v}_{\mathrm{R}} \cdot \mathrm{v}_{\mathrm{N}}}{2}+1\right)\left(1+\frac{\mathrm{t}}{\mathrm{T}}\right)^{\frac{\mathrm{v}_{\mathrm{R}}}{2}-1}} .
$$

1) Siehe Anhang zum 1. Teil. 
Der Übersicht halber wollen wir die Bedeutung aller hier auftretenden Größen rekapitulieren:

$\boldsymbol{\Sigma} \boldsymbol{\nu}=$ Gesamtzahl der kleinen Teilchen zur Zeit $t$

$\mathrm{N}_{\mathbf{0}}=$ ursprüngliche Anzahl der großen Teilchen,

$\mathrm{n}_{\mathbf{0}}=$ ursprüngliche Anzahl der kleinen Teilchen,

$V_{N}=\frac{N_{0}}{n_{0}}=$ Verhältnis der ursprünglichen Teilchenzahlen,

$\mathrm{V}_{\mathrm{R}}=\frac{\mathrm{R}}{\mathrm{r}}=\begin{gathered}\text { Radienverhältnis der beiden Teilchensorten im Ausgangs- } \\ \text { kolloid, }\end{gathered}$

$\mathrm{T}=\frac{1}{\mathrm{~N}_{0} \mathrm{~W}}=$ Koagulationszeit der großen Teilchen.

Ein Vergleich mit Gleichung (13) zeigt, daß der erste Faktor in Formel (18) die Gesamtzahl der großen Teilchen zur Zeit $t$ darstellt.

Wir betrachten nun als erstes Beispiel den Fall, daß ursprünglich gleichviel große wie kleine Teilchen im Kolloid sind, d. h.:

$$
\mathrm{N}_{0}=\mathrm{n}_{\mathrm{0}} \quad \mathrm{V}_{\mathrm{N}}=1 \text {. }
$$

In diesem Falle wird nach Formel (18) die Gesamtzahl der kleinen Teilchen zur Zeit $t$

$$
\sum \nu=\frac{\mathrm{N}_{0}}{\left(1+\frac{\mathrm{t}}{\mathrm{T}}\right)} \cdot \frac{\frac{\mathrm{V}_{\mathrm{R}}}{2}}{\left(\frac{\mathrm{V}_{\mathrm{R}}}{2}+1\right)\left(1+\frac{\mathrm{t}}{\mathrm{T}}\right) \frac{\mathrm{V}_{\mathrm{R}}}{\frac{-2}{2}-1}}
$$

Würden sich nur die kleinen Teilchen im Kolloid befinden, so wäre diese Anzahl nach v. Smoluchowskis Theorie

$$
\sum \nu=\frac{n_{0}}{1+\frac{t}{T}}=\frac{N_{0}}{1+\frac{t}{T}}
$$

Ein Vergleich dieser beiden Ausdrücke zeigt, daß in unserem Falle die Zahl der kleinen. Teilchen mit wachsendem $t$ bedeutend rascher abnimmt, denn der Quotient $\frac{\sum_{v}}{\bar{\Sigma} v}$ wird sehr rasch verschwindend klein, wie $\frac{1}{\mathrm{v}_{\mathrm{R}}}$, sobald $\mathrm{V}_{\mathrm{R}}$ einigermaßen groß ist. Das heißt aber, $\left(1+\frac{t^{T}}{\mathrm{~T}}\right)^{\mathrm{v}_{\mathrm{R}}}$

daß die kleinen Teilchen infolge der Anwesenheit der größern bedeutend rascher verschwinden als ohne dieselben.

Diesen raschen Abfall der Teilchenzahl $\sum v$ zeigt Fig. 1. Die Kurven sind berechnet für $\mathrm{N}_{0}=\mathrm{n}_{0}$. Als Abszisse ist aufgetragen $\tau=\frac{\mathrm{t}}{\mathrm{T}}$, das 
ist die Zeit, ausgedrückt in Vielfachen der Koagulationszeit $\mathrm{T}=\frac{1}{\mathrm{~N}_{0} \mathrm{~W}}$ der großen Teilchen; als Ordinate $\frac{\Sigma v}{n_{0}}$, das ist die prozentuale Anzahl der kleinen Teilchen, gemessen an der ursprünglichen Anzahl. Die ausgezogenen Kurven geben die Änderung von $\sum v$ für Werte von $V_{R}=10$, 20,100, während die gestrichelte Kurve die Änderung von $\bar{\Sigma} v$ darstellt, wenn die großen Teilchen nicht im Kolloid wären.

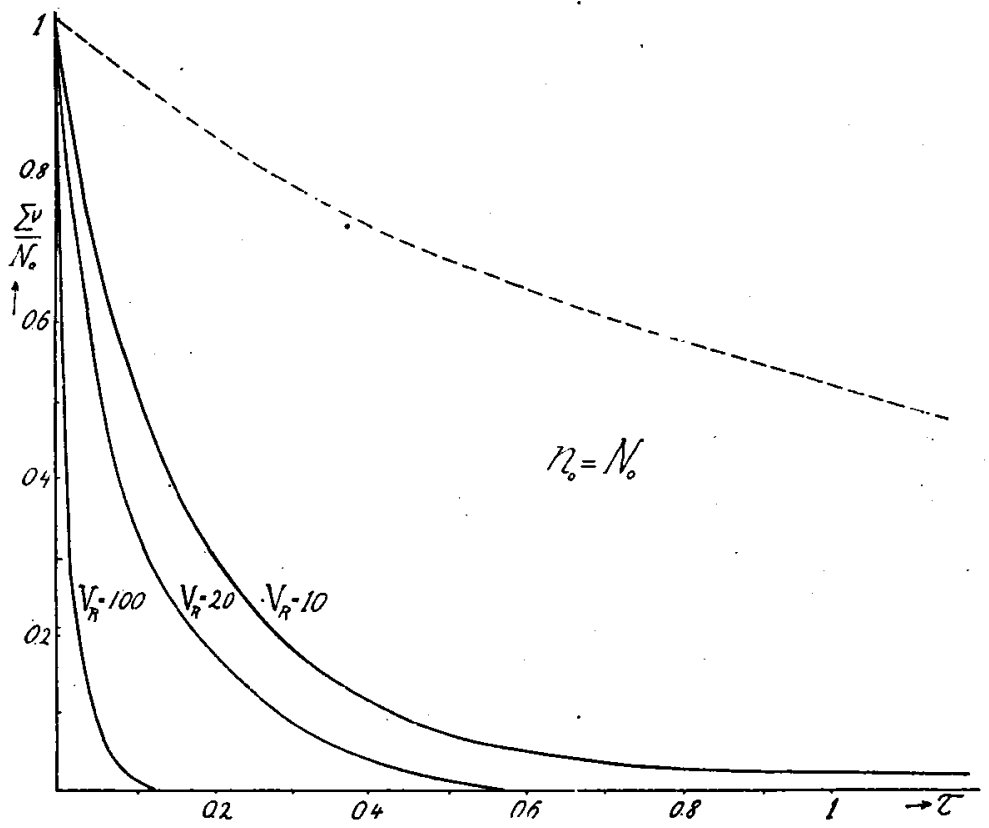

Fig. 1.

In analoger Weise ist in den Fig. 2. und 3 der Abfall der kleinen Teilchen dargestellt für die Anfangsbedingungen $n_{0}=10 N_{0}$ resp. $\mathrm{n}_{0}=\frac{\mathrm{N}_{0}}{10}$ für die Radienverhältnisse $\mathrm{V}_{\mathrm{R}} \doteq 10$ und $\mathrm{V}_{\mathrm{R}}=20$. Die gestrichelten Kurven zeigen wiederum zum Vergleiche den Abfall, wie er sich nach der v. Smoluchowskischen Theorie ergäbe.

Alle diese Kurven demonstrieren ganz deutlich den von Wiegner und Galecki gefundenen Effekt. Infolge der Anwesenheit der großen Teilchen verschwinden die kleinen Teilchen sehr viel rascher, als sie es sonst täten. Der Einfluß ist um so stärker, je größer die Anzahl der großen Teilchen relativ zur Zahl der kleinen ist. Fast alle kleinen Micellen lagern sich in sehr kurzer Zeit an die großen an; die großen. Micellen spielen die Rolle von Koagulationskeimen. 
Dieses Abfangen der kleinen Teilchen durch die großen geschieht, wie die Figuren zeigen, so rasch, daß in fast allen Fällen schon nach der Zeit $t=T$ praktisch überhaupt keine kleinen Teilchen mehr existieren. Dieser Umstand berechtigt uns zu behaupten, daß unsere Formeln (13), (16) und (18), die ursprünglich nur für den Anfang der Koagulation richtig waren, auch für deren weiteren Verlauf anwendbar sind.

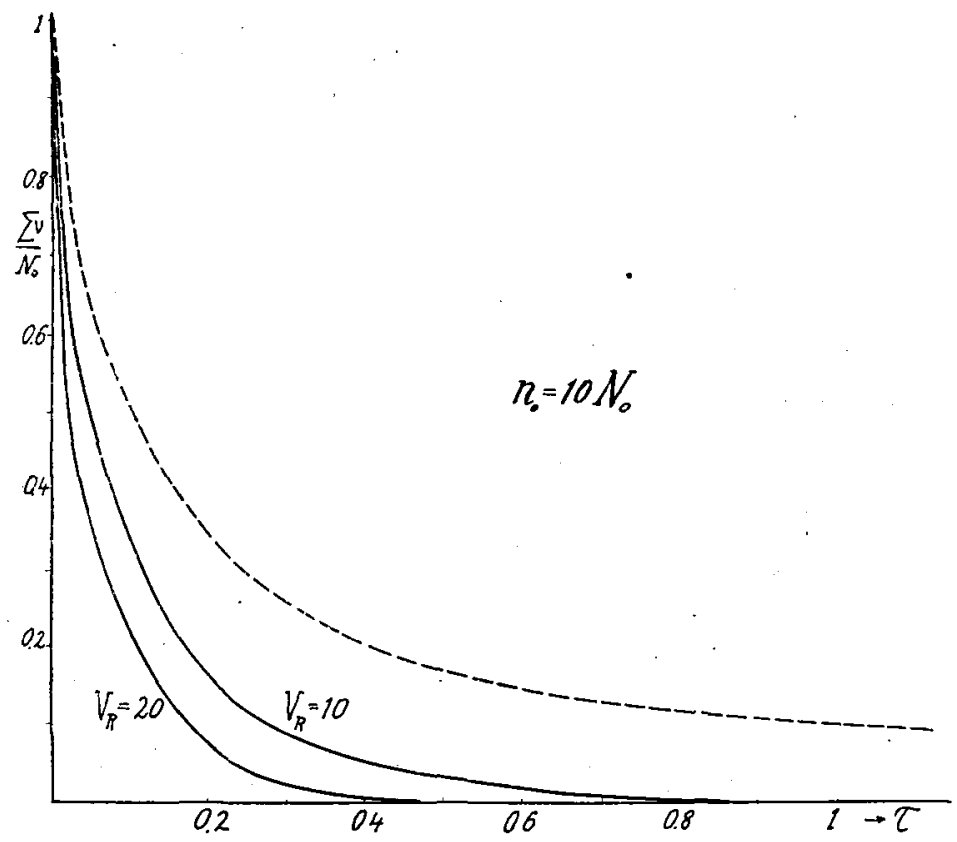

Fig. 2.

Denn wir haben ja zur Lösung der Differentialgleichungen nur vorausgesetzt, daß wir die Bildung von großen Teilchen aus lauter kleinen am Anfang vernachlässigen dürfen. Da es sich jetzt aber zeigt, da $B$ für spätere Zeiten praktisch überhaupt keine kleinen Teilchen mehr existieren, so trifft diese Annahme auch für größere Zeiten $t \mathrm{zu}$. Somit ist der Ausdruck (13) für $\sum N$ und damit eo ipso auch der (16) für $\sum \boldsymbol{\nu}$ praktisch für den ganzen Koagulationsverlauf gültig.

Damit dürfte dieser Effekt von Wiegner und Galecki auch vom theoretischen Standpunkt aus gesichert sein. Herrn Tuorilal) im Agrikulturchemischen Institut der E. T. H. in Zürich ist es im Anschluß an diese Arbeit gelungen, die Schlußformeln (13), (16) resp. (18)

1) Tuorila, Züricher Dissertation 1926 (Kolloidchem. Beih. 22, 191 [1926]); siehe auch G. Wiegner, Koll.-Zeitschr. 38, 3 (1926). 
unserer Theorie direkt an einer Mischung von grobdispersem und amikronischem Goldsol durch ultramikroskopische Auszählungen zu prüfen und mit denkbar bester Genauigkeit zu bestätigen. Er und Herr Prof. Dr. G. Wiegner ${ }^{1}$ ) haben die Diskussion der Endformeln noch weiter geführt und überall eine sehr gute Übereinstimmung mit der Erfahrung gef:nnden.

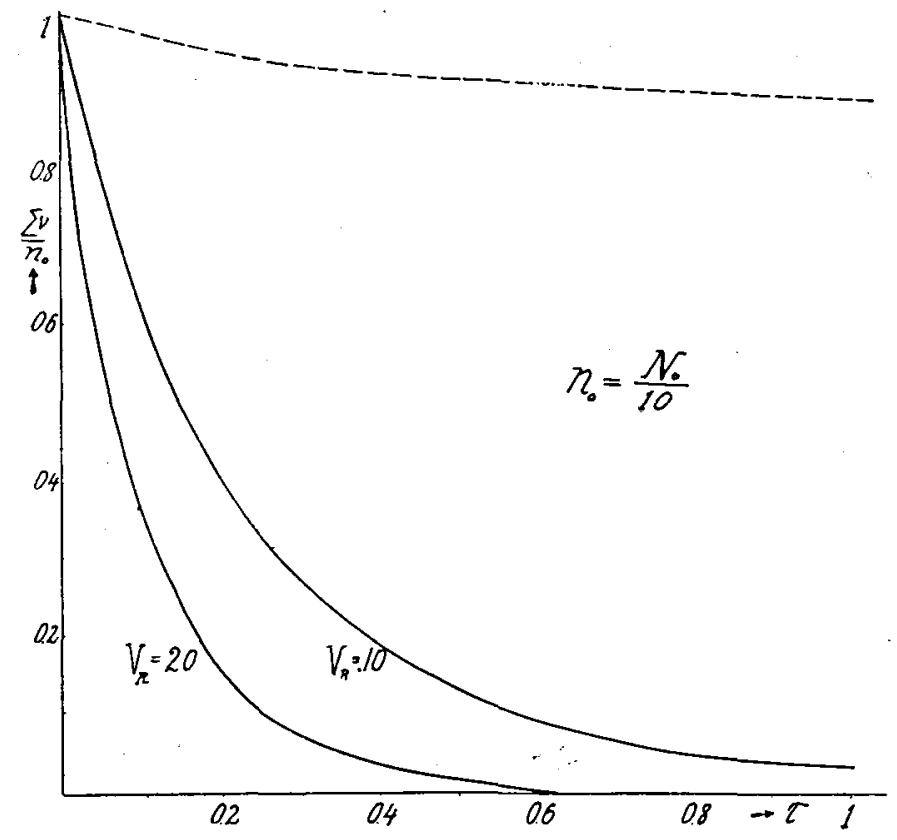

Fig. 3.

Nachdem man $\boldsymbol{\Sigma} N$ und $\boldsymbol{\Sigma} \boldsymbol{v}$ kennt, ist es natürlich einfach, alle Differentialgleichungen (10) sukzessive zu lösen; für $\boldsymbol{\nu}_{1}$ erhält man so den Ausdruck:

$$
\nu_{1}=\mathrm{N}_{0}\left(1+\frac{\mathrm{t}}{\mathrm{T}}\right)^{\frac{2 \lambda}{\lambda+1}} \frac{\mathrm{V}_{\mathrm{N}} \lambda^{2}}{\left[\left(\mathrm{~V}_{\mathrm{N}} \lambda+1\right)\left(1+\frac{\mathrm{t}}{\mathrm{T}}\right)^{\lambda}-1\right]^{2}}
$$

Fig. 4 zeigt, daß die Kurven für $\boldsymbol{\nu}_{\mathrm{i}}$ innerhalb der Kurve für $\boldsymbol{\Sigma} \boldsymbol{v}$ gan $z$ analog verlaufen wie beim monodispersen Fall.

Unser Effekt erklärt auch die systematischen Abweichungen der experimentellen Kurven von Westgren und Reitstötter ${ }^{2}$ ) von der

1) G. Wiegner u. H. Müller, Koll.-Zeitschr. 38, 3 (1926). Hier findet sich auch ein Referat der vorliegenden Arbeit.

2) Loc, cit. S. 5. 
v. Smoluchowskischen Theorie. Die Versuchsreihen zeigen nämlich einen regelmäBigen Gang des Wertes von $\frac{A_{i i}}{\rho_{i}}$. Dieser fällt stets von etwa 3,4 am Anfang der Koagulation bis auf 2 und manchmal noch darunter bis 1,7 für lange Koagulationszeiten. Es ist aber sehr unwahrscheinlich, daß $\frac{A}{\rho}$ nicht eine Konstante ist. Wäre nämlich $\frac{A}{\rho}>2$, so würde

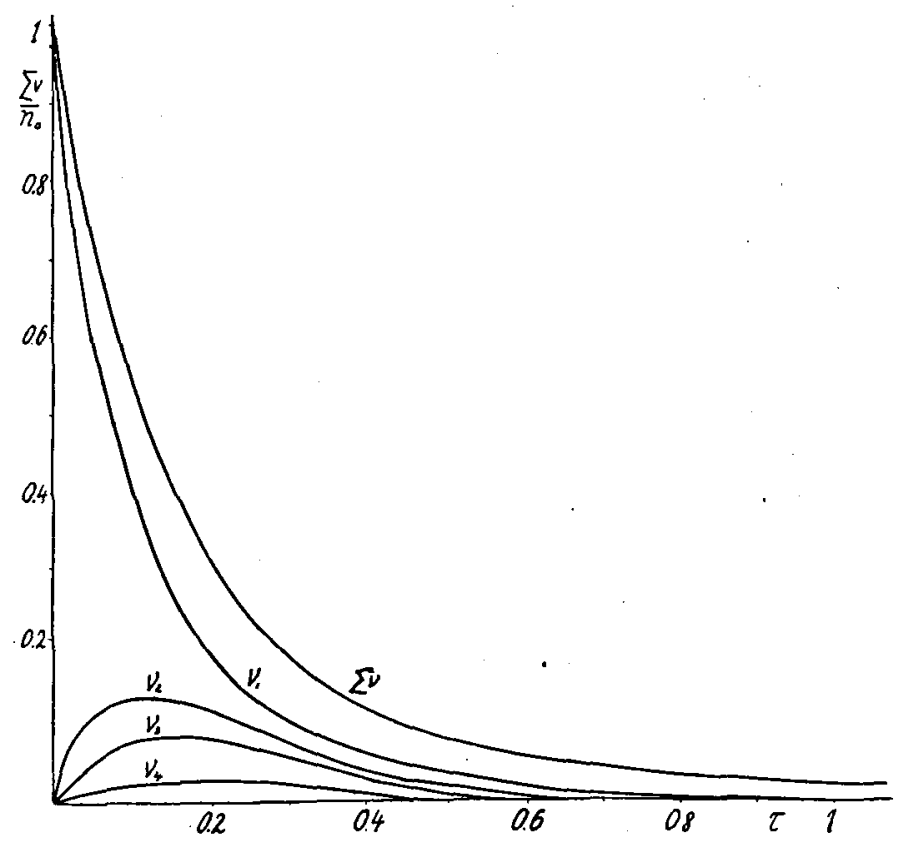

Fig. 4.

das heißen, daß die Micellen einander schon vor der Berührung anziehen auf Abstände von der Größenordnung der Teilchenradien. Die einzigen Kräfte, die man für das Zusammenhaften der Micellen verantwortlich machen kann, sind wohl die Molekularkräfte der Oberflächenatome. Die Wirkungssphäre dieser Kräfte ist aber nur von der Größenordnung der Moleküldurchmesser, also bedeutend kleiner als die Teilchenradien. Es kann also der Wert von $\frac{A}{\varrho}$ nur unwesentlich von 2 abweichen.

Nun entspricht aber ein Wert von $\frac{A}{\varrho}>2$ einem zu steilen Abfall der Kurve für $\sum \nu$. Da dieser zu steile Abfall nur am Anfang der Koagulation auftritt, so kann man ihn jetzt leicht mit der Annahme erklären, $d a B$ in den betreffenden Solen, so einheitlich sie auch schienen, die Teilchengröße doch nicht ideal konstant war. Die gleiche Erklärung 
dürfte auch für spätere Versuche, die noch größere Werte von $\frac{\mathrm{A}}{\varrho}$ ergaben, zutreffen.

Es dürfte damit unsere eingangs gemachte Hypothese, daß für die Koagulation der Micellen Berührung derselben notwendig ist, aus den Versuchen von Westgren und Reitstötter strenger begründet sein.

Freundlich ${ }^{1}$ ) hat darauf hingewiesen, daß der Wiegnersche Effekt die Möglichkeit bietet, den autokatalytischen Verlauf der langsamen Koagulation zu erklären. Wenn nämlich die großen Micellen als Koagulationskeime wirken, so muß sich die Flockung in jedem Sol beschleunigen, sobald sich einmal eine genügend große Anzahl größerer Komplexe gebildet hat. Für die rasche Koagulation dürfte daher dieser Effekt nach unseren Ausführungen bestimmt erwartet werden. Er spielt aber hier keine so große Rolle, weil der Flockungsprozeß sowieso in kurzer Zeit vollendet ist. Er könnte höchstens an einem ganz feindispersen Sol von geringer Konzentration beobachtet werden.

Eine Verallgemeinerung unserer Betrachtung auf die langsame Koagulation ist aber nicht ohne weiteres erlaubt. Die langsame Koagulation hängt ganz wesentlich von der Elektrolytkonzentration ab, d. h. von dem Potential der Micellen gegenüber der Lösung. Der Wahrscheinlichkeitsfaktor $\mathrm{W}$ eines Zusammenstoßes wird daher eine Funktion dieses Potentials. v. Smoluchowski²) hat nun angenommen, da $B$ bei gegebener Elektrolytkonzentration dieses Potential einen konstanten Wert hat, so daß die Koagulationsgleichung der langsamen Koagulation sich von derjenigen der raschen Flockung nur dadurch unterscheidet, $\mathrm{daB}$ an Stelle von $W$ eine kleinere aber konstante Wahrscheinlichkeit $W^{\prime}$ tritt. Er kommt so zu dem Satz, daß alle Koagulationskurven zueinander affin sein müssen.

Es ist nun aber nicht ausgeschlossen ${ }^{3}$ ), da $B$ das Potential der Micellen sich im Verlaufe der Koagulation ändern kann, mit anderen Worten, daß das Potential auch eine Funktion der Größe der Micelle ist. Wir haben Gründe, auf die wir an anderer Stelle) zurückkommen werden, dies anzunehmen. Wenn diese Vermutung zutrifft, so müssen die Koagulationskurven nicht mehr affin sein, und vor allem können

1) H. Freundlich, Kapillarchemie (2. Aufl. 1922), 604.

2) Loc. cit. S. 5.

3) Beobachtungen in dieser Richtung liegen bis heute keine vor. Die wenigen Messungen von Galecki (Zeitschr. f. anorg. Chem. 74, 174 [1912]) an nicht dialysierten Solen liefern keine sichern Anhaltspunkte.

4) Siehe im zweiten Teil der Arbeit am Schlusse. 
wir, um die Keimwirkung der großen Micellen zu berechnen, für $\gamma$ keine so einfache Funktion der Teilchenradien mehr annehmen. Die Rechnung wird dann bedeutend kompliziertcr.

\section{Anhang zum ersten Teil.}

\section{Die Lösung der Differentialgleichung.}

Setzt man in (14) $\Sigma \nu=u . v$, so läßt sich schreiben:

$$
\frac{1}{2 W}\left(u v^{\prime}+v u^{\prime}\right)+\frac{1}{2} u^{2} v^{2}+\gamma \cdot u v \frac{N_{0}}{1+\frac{t}{T}}=0 .
$$

Diese Gleichung trennt man in zwei (siehe z. B. Biberbach, Differentialgleichungen, S. 10. Springer, Berlin 1923):

$$
\left\{\begin{aligned}
u\left(\frac{v^{\prime}}{2 W}+\gamma N_{0} \frac{v}{1+\frac{t}{T}}\right) & =0 \\
\frac{1}{2} \bar{w}^{\prime} u^{\prime}+\frac{u^{2} v^{2}}{2} & =0 .
\end{aligned}\right.
$$

Die erste liefert bei Integration

$$
\frac{\mathrm{v}^{\prime}}{\mathrm{v}}=-2 \mathrm{~W} \gamma \mathrm{N}_{0} \frac{1}{1+\frac{\mathrm{\tau}}{\mathrm{T}}},
$$

woraus $\quad \lg \mathrm{v}=-2 \mathrm{WN}_{0} \mathrm{~T} \gamma \cdot \lg \left(1+\frac{\mathrm{t}}{\mathrm{T}}\right)+\lg \mathrm{v}_{0}$.

Da $\mathrm{T}=\frac{1}{\mathrm{~N}_{0} \mathrm{~W}}$, hat man somit

$$
\mathrm{v}=\mathrm{v}_{0}\left(1+\frac{\mathrm{t}}{\mathrm{T}}\right)^{-2 \gamma}
$$

Die zweite Gleichung lief ert durch Einsetzen von v

$$
-\frac{\mathrm{u}^{\prime}}{\mathrm{u}^{2}}=\mathrm{Wv}=\mathrm{Wv}_{0}\left(1+\frac{\mathrm{t}}{\mathrm{T}}\right)^{-2 y},
$$

woraus durch Integration

$$
\frac{1}{\mathrm{u}}=\frac{\mathrm{Wv}_{0} \mathrm{~T}}{(-2 \gamma+1)}\left(1+\frac{\mathrm{t}}{\mathrm{T}}\right)^{-2 \gamma+1}+\mathrm{c} .
$$

Berücksichtigt man WT $=\frac{1}{\mathrm{~N}_{0}^{-}}$und setzt die Integrationskonstante $c=\frac{C v_{0}}{N_{0}(-2 \gamma+1)}$, so wird:

$$
\begin{aligned}
& c=\overline{\mathrm{N}}_{0}(-2 \gamma+1) \\
& \text { Somit } \quad \sum \nu=\mathrm{uv}=\frac{-\mathrm{N}_{0}(2 \gamma-1)}{\mathrm{v}_{0}\left[\left(1+\frac{\mathrm{t}}{\mathrm{T}}\right)^{-2 \gamma+1}+\mathrm{C}\right]} \\
& \quad \sum \nu=\frac{\mathrm{N}_{0}(2 \gamma-1)}{\left(1+\frac{\mathrm{t}}{\mathrm{T}}\right)^{2 \gamma}\left[-c-\left(1+\frac{\mathrm{t}}{\mathrm{T}}\right)^{2 \gamma-1}\right]}
\end{aligned}
$$


Setzen wir

$$
(2 \gamma-1)=\lambda
$$

so wird:

$$
\sum \nu=\frac{\mathrm{N}_{0}}{\left(1+\frac{\mathrm{t}}{\mathrm{T}}\right)} \cdot \frac{\lambda}{\left[-\mathrm{c}\left(1+\frac{\mathrm{t}}{\mathrm{T}}\right)^{\lambda}-1\right]} \cdot
$$

Die Anfangsbedingung lautet: Für $t=0$ ist $\Sigma v=n_{0}$, es muß also

$$
\mathrm{n}_{0}=\frac{\mathrm{N}_{0} \lambda}{-\mathrm{c}-1}, \text { woraus } \mathrm{c}=-\left(\frac{\mathrm{N}_{0}}{\mathrm{n}_{0}} \lambda+1\right) \text {. }
$$

Führen wir diesen Wert von $c$ ein, und erweitern die Gleichung mit $n_{0}$, so erhalten wir die Endformel (16):

$$
\sum v=\frac{\mathrm{N}_{0}}{1+\frac{\mathrm{t}}{\mathrm{T}}} \frac{2 \mathrm{n}_{0}}{\left[\left(\mathrm{~N}_{0} \lambda+\mathrm{n}_{0}\right)\left(1+\frac{\mathrm{t}}{\mathrm{T}}\right)^{\lambda}-\mathrm{n}_{0}\right]} .
$$

Nachweis der Grenzfälle.

1. Für $\mathrm{N}_{0}=0$ muß (16) in die v.S moluchowskische Formel übergehen, die für diesen Fall lautet:

$$
\sum \nu=\frac{n_{0}}{1+n_{0} W t} .
$$

Es ist: $\quad\left(\sum v\right)_{N_{0}=0}=\lambda n_{0}\left[\frac{N_{0}}{\left(N_{0} \lambda+n_{0}\right)\left(1+\frac{t}{T}\right)^{\lambda}-n_{0}}\right]_{N=0}$

Die Klammer stellt einen Ausdruck $\frac{0}{0}$ dar, somit erhält man durch Differentiation von Zähler und Nenner nach $N_{0}$ (wobei $T=\frac{1}{N_{0} W}$ ):

$$
\begin{gathered}
\left(\sum \nu\right)_{\mathrm{N}_{0}=0}=\lambda \mathrm{n}_{0} \frac{1}{\left[\left(\mathrm{~N}_{0} \lambda+\mathrm{n}_{0}\right) \lambda\left(1+\frac{\mathrm{t}}{\mathrm{T}}\right)^{\lambda-1} \mathrm{Wt}+\lambda\left(1+\frac{\mathrm{t}}{\mathrm{T}}\right)^{\lambda}\right]_{\mathrm{N}=0}} \\
=\frac{\lambda \mathrm{n}_{0}}{\mathrm{n}_{0} \lambda \overline{\mathrm{Wt}}+\lambda}=\frac{\mathrm{n}_{0}}{1+\mathrm{n}_{0} \mathrm{Wt}} \text { w. z. b. w. }
\end{gathered}
$$

2. Für $V_{R}=1$ wird nach (17) $\lambda=1$, somit:

$$
\sum v=\frac{\mathrm{N}_{0}}{1+\frac{\mathrm{t}}{\mathrm{T}}} \cdot \frac{\mathrm{n}_{0}}{\left(\mathrm{~N}_{0}+\mathrm{n}_{0}\right)\left(1+\frac{\mathrm{t}}{\mathrm{T}}\right)-\mathrm{n}_{0}},
$$

während nach (13):

$$
\sum N=\frac{N_{0}}{1+\frac{t}{T}}
$$

also die Summe

$$
\sum \nu+\sum N=\frac{N_{0}}{1+\frac{t}{T}} \cdot \frac{n_{0}+\left(N_{0}+n_{0}\right)\left(1+\frac{t}{T}\right)-n_{0}}{\left(N_{0}+n_{0}\right)\left(1+\frac{t}{T}\right)-n_{0}}=\frac{\left(N_{0}+n_{0}\right)}{\left(1+\frac{n_{0}}{N_{0}}\right)\left(1+N_{0} W t-\frac{n_{0}}{N_{0}}\right.}
$$


Der: Nenner liefert ausmultipliziert:

$1+\mathrm{N}_{0} \mathrm{Wt}+\frac{\mathrm{n}_{0}}{\mathrm{~N}_{0}}+\mathrm{n}_{0} \mathrm{Wt}-\frac{\mathrm{n}_{0}}{\mathrm{~N}_{0}}=1+\left(\mathrm{n}_{0}+\mathrm{N}_{0}\right) \mathrm{Wt}$

Somit:

$$
\sum \nu+\sum N=\frac{\left(N_{0}+n_{0}\right)}{1+\left(N_{0}+n_{0}\right) W_{t}}
$$

das ist aber gerade die Koagulationsformel eines monodispersen Systems mit einer ursprünglichen Teilchenzahl $\left(\mathrm{N}_{0}+\mathrm{n}_{0}\right)$.

Die Möglichkeit dieses lețzteren Überganges mußte nach. unserer Rechnung nicht notwendig folgen, denn diese setzt wesentlich voraus, daß $V_{R} \gg$ 1. Die Möglichkeit des Überganges dürfte dafür sprechen, daß Formel (16) allgemeine Gültigkeit hat, auch fiür kleineres $V_{R}$.

\section{Zweiter Teil.}

\section{Zur Theorie des elektrokinetischen Potentials.}

(Mit 10 Figuren.)

I.

Die elektrische Doppelschicht um die Micellen, welche die Ursache der Stabilität des kolloiden Zustandes ist, bedingt auch die elektraphoretischen Erscheinungen, vor allem die sogenannte-Kataphorese, das ist die Strömung eines Kolloids in einem elektrischen Potentialgefälle: Ist $\xi$ der durch die Doppelschicht hervorgerufene Potentialsprung zwischen der Micelle und dem Lösungsmittel, so ergibt sich nach Debye und Hückeli1) die kataphoretische Wanderungsgeschwindigkeit u eines kugelförmigen Teilchens in einem Felde von der elek. trischen Feldstärke $\mathbb{E}$ nach der Formel

$$
\mathrm{u}=\frac{\boldsymbol{\xi} \mathrm{D}}{6 \pi^{\prime} \eta} \mathrm{E}
$$

Darin ist $\mathrm{D}=$ die Dielektrizitätskonstante des Lösungsmittels, $\eta=$, Viskositätskonstante

Diese Formel erlaubt aus der mikroskopisch oder makroskopisch gemessenen Geschwindigkeit u den Potentialsprung $\xi$ zu berechnen. Ein Vergleich mit Koagulationsexperimenten zeigt, daß dieses Potential tatsächlich ein Maß für die Stabilität eines Kolloides bildet.

Wie die Koagulationsgeschwindigkeit, so hängt auch das elektro. kinetische Potential sehr stark von der Elektrolytkonzentration $\mathrm{c}$ ab.

1) P. Debye u. E. Hückel, Physik. Zeitschr. 25, 49 (1924); E. Hückel, Physik. Zeitschr. 25, 204 (1924). 
Diese $\xi(c)$-Kurven zeigen aber einen sehr unregelmäßigen Verlauf und sind für verschiedene Elektrolyte und verschiedene Kolloide stark verschieden. Im allgemeinsten Falle ist der Verlauf der folgende:

Für sehr kleine Elektrolytkonzentrationen (1-10 Mikromol/Liter) wächst das Potential mit der Konzentration an, erreicht ein Maximum und fällt dann regelmäßig ab, bis im sogenannten, ,isoelektrischen Punkt" das Potential verschwindet. Die Konzentration im isoelektrischen Punkt ist identisch mit derjenigen, die für rasche Koagulation notwendig ist. Eine noch höhere Konzentration kann das Potential sogar noch weiter erniedrigen, bis zu einem maximalen negativen Wert. Man spricht dann von Umladung des Teilchens. Dieses "umgeladene“ Potential erreicht gewöhnlich keinen so hohen Wert wie das ursprüngliche, und verschwindet wiederum für noch höhere Kunzentrationen.

Die auftretenden Potentiale $\xi$ sind von der Größenordnung 10 bis 100 Millivolt; die zur vollständigen Entladung nötigen Elektrolytkonzentrationen vatiieren $z$ wischen 10 und 1000 Mikromol pro Liter.

Dieser allgemeinste Verlauf wird aber nicht in allen Fällen befolgt: Das charakteristische Merkmal aller Kurven ist nur der Abfall des Potentials von dem ursprünglichen Wert auf Null. H. Fír eu nd lich hat diesen Abfall empirisch durch eine Formel

$$
\xi=a \ln \frac{b}{c}
$$

dargestellt, in der a und b empirische Konstanten sind. Die Steilheit des Abfalls hängt ganz wesentlich von der Wertigkeit desjenigen Ions ab, das den entgegengesetzten Ladungssinn als der der Micelle hat. Ein mehrwertiges Ion erzeugt einen ganz bedeutend steileren A Abfall. Dás Verhältnis der Konzentrationen ein-, zwei-, drei-, vierwertiger lonen, die nötig sind, um die gleiche Potentialerniedrigung hervorzurufen, ist ungefähr

$$
c_{1}: c_{2}: c_{3}: c_{4}=3000: 80: 2: 1 \text {. }
$$

Es scheint daneben auch ein kleiner Einfluß der Wertigkeit des gleichgeladenen Ions zu existieren.

Die andern Effekte, anfänglicher Anstieg und Umladung, treten nicht immer auf. Inre Existenz scheint von dem chemischen Charakter der Ionen und der Micellsubstanz abzuhängen.

Im großen ganzen sind die Kurven durch die Wertigkeit der Ionen bestimmt. Nur im Falle solcher Ionen, die auch aus andern Gründen (Theorie der Elektrolyte) als, stark hydratisiert betrachtet jwerden müssen, vollzieht sich der Potentialabfall bedeutend langsamer. " 
Zur Erklärung dieser $\boldsymbol{\xi}(\mathrm{c})$-Kurven existieren zwei verschiedene Hypothesen:

\section{Die Adsorptionstheorie.}

Diese bis heute meist akzeptierte Theorie (siehe z. B. Freundlich, Kapillarchemie) erklärt den Potentialabfall dadurch, daß bei der Erhöhung der Elektrolytkonzentration die entgegengesetzt geladenen Ionen sich an das Kolloidteilchen anlagern und dadurch die Micelle „,entladen“. Indem man diese Anlagerung als eine gewöhnliche Adsorption betrachtet und dafür die aus der Langmuirschen Theorie der Adsorption von neutralen Gasmolekülen an feste Körper bekannte Adsorptionsisotherme setzt, kann man den Abfall des Potentials gemäß einer logarithmischen Funktion verstehen. Es muß aber bemerkt werden, daß es noch keineswegs sichersteht, ob man hier von Adsorption im gewöhnlichen Sinne sprechen darf, und daher die Anwendung der Langmuirschen Formel berechtigt ist. Wir kennen bis heute die Ursache der Aufladung der Micellen noch nicht; es ist möglich, daß dabei chemische Affinitäten (im Sinne der Theorie von Pauli) eine Rolle spielen. Es dürfte zwar aus anderen Versuchen (Färbung der Micellen und chemische Analyse der Koagulate) sicherstehen, daß eine solche Anlagerung der Elektrolytionen stattfindet. Sie muß auf jeden Fall in all den Fällen angenommen werden, wo sich die Teilchen umladen. Es muß dabei allerdings auch die Möglichkeit offengelassen werden, daß auch eine Anlagerung der gleichgeladenen Ionen statthaben kann. Dafür spricht nicht nur der anfängliche Anstieg des Potentials bei den kleinen Konzentrationen und die Entladung der „umgeladenen" Teilchen, sondern auch Experimente über den Einfluß der Konzentration der Kolloide auf die Koagulationskonzentrationen. ${ }^{2}$ )

Der charakteristische Einfluß der Wertigkeit der Ionen wird im Rahmen dieser Theorie durch die Zusatzhypothese erklärt, daß das "Adsorptionsvermögen" für verschiedenwertige Ionen gleich groß ist, eine Annahme, die wohl kaum als physikalisch evident betrachtet werden kann.

Diese Theorie erklärt also die Variation des Potentials nur durch Änderung der Ladung der Doppelschicht und sieht vollkommen davon $a b$, daß auch eine Änderung der Doppelschichtdicke den gleichen Effekt hervorrufen kann. Sie betrachtet die Doppelschichtdicke als

1) H. Freundlich, Kapillarchemie (2. Aufl. Leipzig 1922), 365.

2) S. Gosh u N. R. Dhar, Koll.-Zeitschr. 36, 129 (1925). 
eine Konstante. Es dürfte bei der Unkenntnis der "adsorbierenden“" Kräfte schwer fallen, auf Grund dieser Theorie die $\xi$ (c)-Kurven theoretisch vollkommen zu verstehen. Sicherlich reicht die Annahme der Anlagerung von nur entgegengesetzt geladenen Ionen nicht aus. Ebenso scheint eine Erklärung des Einflusses der Hydratation nicht möglich, ohne Einführung weiterer Zusatzhypothesen.

\section{Die Theorie der diffusen Doppelschicht.}

Diese zuerst von Gouyl) entwickelte. Theorie, an die sich Arbeiten von (hapman'), Herzfeld ${ }^{3}$ ), Gyemant ${ }^{4}$ ) u. a. anschließen, versucht im wesentlichen die ganze Änderung des Potentials durch Änderung der Doppelschichtdicke zu erklären. Die Theorie bezieht sich $z$ war ursprünglich nicht auf das bei der Kataphorese wirksame elektrokinetische Potential $\xi$, sondern auf das aus kapillarelektrischen Effekten bestimmte Phasengrenzpotential $\varepsilon$ zwischen einem festen Körper und einem Elektrolyten. Diese beiden Potentiale sind zwar im allgemeinen nicht identisch (wir kommen darauf zurück); wir wollen aber vorläufig für eine nur prinzipielle Überlegung diesen Unterschied vernachlässigen. Gouy hat darauf aufmerksam gemacht, daß man sich die Doppelschicht um ein Kolloidteilchen nicht vorstellen darf als ein starres System zweier entgegengesetzt geladener Schichten. Über die Konstitution der inneren ,adsorbierten" Schicht wissen wir zwar nichts. $\mathrm{Da}$ diese Belegung aber starr mit dem Teilchen verbunden ist und da die Anzahl der angelagerten Ionen stets 10000 bis 100000 mal kleiner ist als die Zahl der Oberflächenatome des festen Körpers, so dürfen wir sie wohl als monomolekular betrachten. Die äußere Belegung der Doppelschicht kommt nun so zustande, daß die Ladung der Micelle die entgegengesetzt geladenen Ionen des Elektrolyten elektrostatisch anzieht und die gleichgeladenen Ionen abstößt. Gegen diese trennenden elektrischen Kräfte wirkt aber die mischende Wirkung der Temperaturbewegung, deren Energie gerade von der gleichen Größenordnung ist, wie die der Coulombschen Kräfte. Unter der Wirkung beider Einflüsse stellt sich eine statistische Ionenverteilung ein, derart, daß in der Nähe der Teilchenoberfläche die Zahl der entgegengesetzt geladenen Ionen im zeitlichen Mittel größer, die der gleichgeladenen kleiner ist als in der übrigen Lösung. Es bildet sich so um das Teilchen eine

1) Gouy, Compt. rend. 149, 654 (1909); Journ. de phys. 9, 457 (1910). Annales de physique 7, 129 (1917).

2) Chapmann, Phil. Mag. 25, 654 (1909).

3) Herzfeld, Phys. Zeitschr. 21, 28 (1920).

4) Gyemant, Zeitschr. f. Physik 17, 190 (1923). 
„Atmosphäre" mit einem Ladungsüberschuß. Diese Atmosphäre bildet die äußere Belegung der Doppelschicht. Eine einfache Rechnung zeigt nun, daß die. Dicke dieser „diffusen“"Belegung mit wachsender Elektrolytkonzentration rasch abnimmt. Man findet unter: Annahme einer ebenen Doppelschicht folgende Beziehung zwischen dem Potential $\xi$ und der Konzentration c, im Falle eines binären Elektrolyten mit zwei gleichwertigen Ionen von der Valenz $\mathbf{z}^{1}$ ):

$$
\xi=\frac{2 \mathrm{~A}}{\mathrm{z}} \operatorname{Arcsinh} \frac{\sigma}{2 \alpha \sqrt{\mathrm{c}}} .
$$

Darin bedeutet $\sigma$ die spezifische Oberflächenladung der Micelle. Die Größen $\mathrm{A}$ und $\boldsymbol{\alpha}$ sind Konstante, die durch die Temperatur $\mathrm{T}$ und die Dielektrizitätşkonstante $\mathrm{D}$ des Lösungsmittels vollkommen bestimmt sind. Bezeichnen wir mit e $=4,77: 10^{-10}$ e. 1 . E. die elektrische Elementarladung, mit $\mathrm{k}=1,346 \cdot 10^{-16}$ die Boltzmannsche Konstante, und mißt man die Konzentration $\mathrm{c}$ in Mikromol/Liter, so wird

$$
\begin{aligned}
\mathrm{A} & =\frac{\mathrm{kT}}{\mathrm{e}} \\
\alpha & =\sqrt{\frac{\mathrm{DkT} \cdot 6,06 \cdot 10^{-14}}{2 \pi}} .
\end{aligned}
$$

Für Hydrosolen bei einer Temperatur von $18^{\circ}(T=291, D=81)$ ist

$$
\begin{aligned}
A & =25 \text { Millivolt, } \\
\alpha & =17,65 .
\end{aligned}
$$

Diese Formel gibt tatsächlich einen Abfall der $\xi(c)$-Kurve, der ganz analog verläuft, wie die Freundlichsche Annäherung (2). Während aber in Freundlichs Formel zwei anzupassende Konstanten auftreten, haben. wir in Formel (3) nur noch die Oberflächenladung $\sigma$ als einzige unbestimmte Größe, über die wir noch mit einiger Willkür verfügen können. Um den experimentellen Werten gerecht $\mathrm{zu}$ werden, müssen wir für $\sigma$ einen Wert von etwa 100 el. st. E. annehmen. Ein solcher Wert für $r$ ist vollkommen vernünftig und entspricht allen Anschauungen über die Größe der Micellenladung, denn er entspricht einer Ladung von 250 Elektronen für ein Teilchen von $10^{-5} \mathrm{~cm}$ Radius.

Gouys Theorie der diffusen Doppelschicht kann somit qualitativ den Abfall der $\xi$ (c)-Kurve sehr gut erklären, und zwar unter Annahme einer konstanten Teilchenladung. Die Potentialerniedrigung kommt nur zustande, weil die Dicke der Ionenschicht abnimmt. Man kann

1) Eine Ableitung dieser Formel und die Berechnung der Konstanten geben wir im nächsten Abschnitt. Arc sin h bedeutet die Umkehrfunktion von Sinus Hyberbolicus. 
aus der Theorie auch eine mittlere Dicke der Doppelschicht bereehnen'. Für die in Frage kommenden Konzentrationen voǹ 10-100 Mikrómol erhält man dafür Werte von $10^{-6}$ bis $10^{-5} \mathrm{~cm}$. Da sie somit von der gleichen Größenordnung wie die Micellenradien ist, so kann Formel (3) (die die Teilchenoberfläche als eben, resp. ihre Krümmung als klein gegen die Doppelschichtdicke annimmt) nur als erste Näherung betrachtet werden.

Diese Theorie liefert aber auch die charakteristische Abhängigkeit von der Wertigkeit der Ionen ohne weitere Zusatzhypothese. Aus Formel (3) folgt z. B., daß sich die Konzentrationen ein-, zwei-, 'drei-, vierwertiger Ionen, die das Potential auf 50 Millivolt heruntersetzen, verhalten wie

$$
\begin{aligned}
c_{1}: c_{2}: c_{3}^{\prime}: c_{4} & =\frac{1}{\sin h^{2} 1}: \frac{1}{\sinh ^{2} 2}: \frac{1}{\sinh ^{2} 3}: \frac{1}{\sinh ^{2} 4} \\
& =\frac{1}{1,37}: \frac{1}{13,1}: \frac{1}{100}: \frac{1}{745}=540: 57: 7,45: 1 .
\end{aligned}
$$

Die Übereinstimmung mit der Erfahrung wird durch Berücksichtigung der Krümmung noch bedeutend verbessert.

Diese Tatsache, daß der Potentialabfall und der Einfluß der Wertigkeit des „entladenden" Ions richtig dargestellt werden, dürfte sehr für die Gouysche Theorie sprechen. Es ist auch leicht einzusehen, daß sie auch eine Abhängigkeit von der Wertigkeit des gleichgeladenen Ions ergibt, der aber neben der des „entladenden“ Ions eine kleinere Rolle spielt. Die Theorie kann auch, wie Wiegner ${ }^{1}$ ) gezeigt hat, zum Verständnis des Einflusses der Hydratation (Hof meistersche Reihen) und der Austauschreaktionen dienen.

Die Gouysche Theorie kann aber unmöglich eine Erklärung geben für den anfänglichen Anstieg der $\xi(c)$-Kurven für kleine Konzentrationen und für die Umladungserscheinungen. Es wurde aber schon darauf aufmerksam gemacht, daß diese ,zwei letzteren Phänomene nicht allgemein auftreten.

Wenn man zwischen diesen beiden Auffassungen entscheiden soll, so muß man sich klar sein, daß zwar einerseits die Adsorption in vielen Fällen experimentell sicher nachgewieșen ist; daß aber anderseits der Auffassung der diffusen Doppelschicht schon heute eine solche physikalische Evidenz zukommt, daß man auf diese Vorstellung nicht mehr länger verzichten darf. Diese Idee hat nicht nur in den Gouyschen Arbeiten über die kapillarelektrischen Erscheinungen ihre Tauglichkeit bewiesen, sie ist vor allem auch die Grundlage der Theorie der Elektro-

1) G. Wiegner, Zsigmondy-Festschrift der Koll.Ztg. 36, 341 (1925). 
lyte von Debye und $\mathrm{Hückel}$, durch die alle Anomalien der starken Elektrolyte so glänzend erklärt.wurden. Da sie auch die charakteristischen Eigenschaften der $\xi$ (c)-Kurven richtig wiederzugeben vermag, dürfte es gegeben sein, als wichtigsten Effekt, der immer auftritt und auftreten muß, die Änderung der Doppelschichtdicke für den Abfall des elektrokinetischen Potentials verantwortlich zu machen.

Die Adsorption dagegen, die in den meisten Fällen sicher auch existiert, müssen wir zur Erklärung all der Nebeneffekte, die die Gouysche Theorie nicht erklären kann, wie anfänglicher Anstieg und Umladung, heranziehen. Dies dürfte auch der Natur dieser Effekte, die wesentlich von chemischen Eigenschaften der Ionen abzuhängen scheinen, vollkommen entsprechen. Der Einfluß der Hydratation folgt ebenfalls aus der Theorie der diffusen Doppelschicht, muß aber vorläufig vernachlässigt werden.

Die Berechtigung dieser Kompromißauffassung kann dadurch bestätigt werden, daß es uns gelingt, in all den beobachteten Fällen, in denen die "Nebeneffekte" nicht auftreten, die $\xi$ (c)-Kurven vollkommen darzustellen mit Hilfe der Theorie der diffusen Doppelschicht, unter Annahme einer konstanten Teilchenladung (also ohne jede Adsorption).

Solche Messungen, die nur den regelmäßigen Abfall der $\xi$ (c)Kurven ohne Umladung zeigen; sind gerade in letzter Zeit von Freundlich und $\left.\mathrm{Zeh}^{1}\right)$ und Fried und $\mathrm{Pauli}^{2}$ ) publiziert worden. Freundlich und $\mathrm{Zeh}$ konstatierten diese einfachen Kurven an einem $\mathrm{As}_{2} \mathrm{~S}_{3}$ Sol in einem Elektrolyten, dessen "entladendes" Ion ein großes Komplexion war. Fried und Pauli fanden das Nichtauftreten der Nebeneffekte an ihren reinsten Silbersolen mit $\mathrm{Al}_{2}\left(\mathrm{SO}_{4}\right)_{3}$ als Elektrolyten. Sie fanden auch, daß die Umladungserscheinung sofort auftritt, wenn neben dem $\mathrm{Al}_{2}\left(\mathrm{SO}_{3}\right)_{3}$ noch kleine Mengen (einige Mikromol/Liter) eines Alkalisalzes, z. B. $\mathrm{Na}_{2} \mathrm{CO}_{2}$ in der Lösung sind. Diese beiden Arbeiten gaben den Anstoß zu der vorliegenden Arbeit.

Warum in dem einen Falle Anlagerung von Ionen stattfindet und in anderen Fällen nicht, kann man natürlich nicht erklären, solange man den "Adsorptionsprozeß" nicht physikalisch oder chemisch einwandfrei verstehen kann.

Um zu untersuchen, ob die oben erwähnten Meßreihen mit Hilfe der Gouyschen Theorie ohne Adsorption erklärt werden können,

1) H. Freundlich u. P. Zeh: Zeitschr. f. phys. Chem. 114, $65(1925$;

2) Fried u. Pauli, Koll.Zeitschr. 36, 138 (1925). 
müssen wir vorerst im. nächsten Abschnitt die Theorie der diffusen Doppelschicht um ein kugelförmiges Teilchen näher studieren, und die' Schwierigkeiten, welche der Unterschied zwischen elektrokinetischem Potential $\xi$ und dem Phasengrenzpotential $\varepsilon$ bietet, zu umgehen suchen.

II.

Die ernsthafteste Schwierigkeit für die Anwendung der Gouyschen Theorie der diffusen Doppelschicht dürfte in dem verschiedenen Verhalten des elektrokinetischen Potentials $\xi$ und der Phasengrenzkraft $\varepsilon$ bestehen. Diese beiden Potentiale unterscheiden sich im wesentlichen dadurch, daß $\varepsilon$ in der Regel größer als $\xi$ ist, in keinem Falle aber kleiner, und daß $\varepsilon$.keine Umladungserscheinung zeigt, auch wenn diese für $\xi$ existiert. H. Freundlich ${ }^{1}$ ) und v. Smoluchowski ${ }^{2}$ ) haben dieses verschiedene Verhalten folgendermaßen gedeutet: Das Phasengrenzpotential $\varepsilon$, als statisch aus der Elektrokapillarkurve bestimmtes Potential, ist der totale Potentialsprung $z$ wischen dem Teilchen und der Lösung. Für die elektrokinetischen Effekte dagegen kommt nur ein Teil von $\varepsilon$ in Frage, weil bei der Kataphorese die nächste Umgebung des Teilchens sich mit diesem bewegt. Das elektrokinetische Potential $\xi$ ist daher das Potential, das an der Oberfläche der mit dem Teilchen starr verbundenen Flüssigkeitshaut herrscht. Man versteht so auch im Rahmen der Gouyschen Theorie, daß immer $\xi<\varepsilon$, weil zwischen Micellenoberfläche und "Abreißschicht" schon ein Potentialabfall bestehen muß. Die Tatsache jedoch, daß $\varepsilon$ und $\xi$ sogar verschiedene Vorzeichen annehmen können, ist mit der einfachen Anschauung der diffusen Doppelschicht unvereinbar. Der prinzipiell richtige Weg zur Lösung dieses Dilemmas dürfte wohl durch die Arbeit von O. Stern ${ }^{3}$ ) gegeben worden sein. Stern zerlegt die äußere Doppelschichtsbelegung in eine "starre" Adsorptionsschicht - entsprechend der Langmuirschen monomolekularen Adsorptionsschicht - und eine weiter in die Flüssigkeit reichende diffuse Ionenatmosphäre. Es gelingt ihm dadurch nicht nur das verschiedene Verhalten der $\xi$ - und $\varepsilon$-Potentiale zu verstehen, sondern es erklärt sich auch das anfängliche Ansteigen des $\xi$-Potentials zu einem Maximalwert. ${ }^{4}$ )

1) H. Freundlich, Kapillarchemie (2. Aufl.), 342.

2) v. Smoluchowski, s. Gratz, Handb. d. Elektr. u. Magn. II, 393.

3) O. Stern, Zeitschr. f. Elektrochemie 30, 508 (1924).

4) Dieses Ansteigen zu einem Maximalwert kann man schon verstehen, wenn man in der Formel (3) für das Potential der diffusen Doppelschicht für die Teilchenladung eine Adsorptionsisotherme ansetzt: siehe Gyemant (Zeitschr. f. Physik 17, 190 [1923]) wo Formel (3) mit Rücksicht auf die Kugelform etwas korrigiert ist. 
Da $\xi$ das Potential in der Abreißfläche ist, so spielt für dessen Be- rechnung die Konstitution der inneren Schichten keine Rolle. Wir dürfen aber wohl sicher annehmen, daß, wenn eine starre Langmuirsche Adsorptionsschicht existiert, sich diese mit dem Teilchen bewegt, daß also die Abreißschicht außerhalb des Adsorptionsraumes liegt. Wir setzen also voraus, wie das auch schon von Stern angenommen wird, daß der Potentialabfall außerhalb der Abreißschicht vollkommen mit demjenigen in ciner diffusen Doppelschicht übereinstimmt. Die Berechtigung dieser Annahme kann natülich nur durch den Vergleich ihrer Konsequenzen mit der Erfahrung geprüft werden; sie dürfte aber schon an sich sehr plausibel sein.

Auf Grund dieser Annahme spielen dann für das elektrokinetische Potential die Prozesse in den innersten Schichten nur noch insofern eine Rolle, als sie die Gesamtladung der diffusen Doppelschicht ändern können. Da sie weiter keinen Einfluß ausüben, so genügt es - wenn wir uns nur auf die Theorie des $\xi$-Potentials beschränken -, die elektrische Ladungs- und Potentialverteilung außerhalb der Abreißschicht zu betrachten und erst in zweiter Linie den Einfluß der Ladungsänderung der inneren Schichten zu berücksichtigen. ${ }^{1}$ ).

Wir wollen deshalb in folgendem, wenn wir von „Teilchen" sprechen, darunter stets den Komplex verstehen, der innerhalb der Abreiß. fläche liegt. Für das Kolloidteilchen im engeren Sinne wollen wir den gebräuchlichen Ausdruck "Micelle" benutzen. Wir denken uns das Teilchen als eine Kugel vom Radius $R$; dieser wird mit dem Radius der Micelle bis auf eine Differenz von der Größenordnung der Moleküldurchmesser übereinstimmen.

Die Totalladung des Teilchens bezeichnen wir mit E. Diese Ladung besteht somit aus der Ladung der Micelle, vermindert um die Ladung der inneren Schichten. Alle Änderungen von E wollen wir kurzweg als Änderungen durch „Adsorption“ bezeichnen. Dieser erweiterte Begriff Adsorption umfaßt dànn sowohl Änderungen der Micellenladung als auch Änderungen der äußeren adsorbierten Ionenschicht.

Wir wollen, um einen bestimmten Fall vor Augen zu haben, $E$ als positiv wählen. Für ein negatives Teilchen bleiben dann die folgenden

1) Wir stellen uns damit auf einen anderen Standpunkt als Stern, de für die Theorie des $\xi$-Potentials nur die Adsorptionsänderung betrachtet, indem er die Ladung der diffusen Doppelschicht vernachlässigt. Diese Vernachlässigung ist sicher nicht gestattet, da doch gerade die Existenz und die Ânderung ides Potentials von der. Existenx und Änderung der diffusen Doppelschicht abhängt. Wir glauben daher nicht, daß in der Sternschen Bezeichnungsweise die Behauptung $\eta_{2}<<\eta_{1}$ zutrifft. 
Überlegungen genau dieselben, da mit dem Vorzeichen von $\mathrm{E}$ auch dasjenige von $\xi$ wechselt. Aus $\mathrm{E}$ und $\mathrm{R}$ resultiert eine spezifische Oberflächenladung $\sigma$ des Teilchens

$$
\dot{\sigma}=\frac{E}{4 \pi \dot{\mathrm{R}}^{2}} \cdot
$$

Dieser Ersatz der Ladung $\mathrm{E}$, die tatsächlich eine Raumladung sein wird, durch eine Oberflächenladung $\sigma$ in der Abreißfläche ist nach dem Gausșchen Satze gestattet. Von der Einführung einer Dielektrizitäts. konstanten der inneren Schichten sehen wir ab, da es uns weniger auf den Absolutwert von $\mathrm{E}$ als auf dessen relative Änderungen ankommt.

Wir stehen somit vor der Aufgabe, das Potential eines kugelförmigen Teilchens vom Radius $R$ und der Ladung $E$ zu berechnen, das sich in einer Elektrolytlösung von der Dielektrizitätskonstanten $\mathrm{D}$ befindet, unter der Voraussetzung, daß die Ionen- resp. Potentialverteilung der Differentialgleichung der diffusen Doppeischicht genügt.

Diese Differentialgleichung erhält man bekanntlich auf folgende Weise: Ist $\psi(r)$ das Potential in einem Abstand $r$ vom Teilchenmittelpunkt, so gilt die Poissonsche Gleichung

$$
\Delta \psi=-\frac{4 \pi}{\mathrm{D}} \mathrm{o} \text { : }
$$

Bedenken gegen die Einführung der makroskopischen Dielektrizitätskonstanten $\mathrm{D}$ dürften bier nicht am Platze sein, da bei den Potentialen und Konzentrationen, die bei der Kataphorese in Frage kommen, der gegenseitige mittlere Abstand der Ionen stets bedeutend größer ist als die Größe der Wassermoleküle. Für solch geringe Konzentrationen hat sich auch in Debyes Theorie der Elektrolyte die gewöhnliche Dielektrizitätskonstante des Wassers als tauglich erwiesen.

$\underline{Q}$ ist die elektrische Ladungsdichte im Abstand $r$. Ist $n_{\mathfrak{i}}$ die in diesem Punkte im zeitlichen Mittel auftretende Anzahl der Ionen einer Sorte $\mathrm{i}$ von der. Wertigkeit $z_{\mathrm{i}}\left(z_{\mathrm{i}}\right.$ mit dem algebraischen Vorzeichen, dem Ladungssinn entsprechend), so ist...

$$
\varrho=\sum_{i} n_{i} z_{i} \mathrm{e}
$$

wo $\mathrm{e}=4,77 \cdot 10^{-10}$ el. st. E. die elektrische Elementarladung ist.

Der zeitliche Mittelwert $n_{\mathbf{i}}$ der Ionenzahl läßt sich anderseits nach dem Maxwell-Boltzmannschen Prinzip bestimmen. Ist nämlich $\mathrm{n}_{\mathfrak{i}}^{0}$ die Zahl der Ionen auf $1 \mathrm{ccm}$, die bei Gleichverteilung herrschen würde, so ergibt sich danach (wenn man die Ionen als Punkte auffaßt).

$$
n_{i}=n_{i}^{0} e^{-z_{i} \frac{e \psi}{k T}}
$$


$\mathrm{n}_{1}^{0}$ wird einfach gegeben durch die experimentell gegebenen mittleren Elektrolytkonzentrationen. Ist $c_{i}$ die Konzentration der $\mathrm{i}$-Ionen in Mikromol per Liter, so ist:

$$
\begin{aligned}
\mathrm{n}_{1}^{0} & =\mathrm{c}_{\mathrm{i}} \frac{\mathrm{N}}{10^{+9}}=\mathrm{c}_{\mathrm{i}} \boldsymbol{\nu} \\
(\mathrm{N} & \left.=6,0610^{23}=\text { Avogadro-Zahl }\right) \\
\boldsymbol{v} & =6,06 \cdot 10^{14} .
\end{aligned}
$$

Aus (5), (6), (7) und (8) ernält man dann die gesuchte Differentialgleichung der diffusen Doppelschicht

$$
\Delta \psi=-{ }_{\mathrm{D}}^{4 \pi} \nu \mathrm{e} \sum_{\mathrm{i}} \mathrm{c}_{\mathrm{i}} z_{\mathrm{i}} \mathrm{e}^{-\frac{z_{\mathrm{i}} \circ \psi}{\mathrm{KT}}} .
$$

Wir haben also bei gegebenen $c_{i}, z_{i}$ diese Differentialgleichung für den kugelsymmetrischen Fall zu lösen mit den Randbedingungen, daß in großem Abstand vom Teilchen das Potential und die Feldstärke verschwinden, also:

$$
\text { für } \mathrm{r}=\infty, \quad \psi=0, \quad \frac{\mathrm{d} \psi}{\mathrm{d} \mathrm{r}}=0,
$$

und daß die Gesamtladung der diffusen Doppelschicht gerade die Teilchenladung kompensiert, d. h.:

$$
\text { für } \mathrm{r}=\mathrm{R} \quad \frac{\mathrm{D}}{4 \pi} \frac{\mathrm{d} \psi}{\mathrm{dr}}=-\sigma .
$$

Das gesuchte Potential ergibt sich dann als der Wert von $\psi$ im Abstand R:

$$
\xi=\psi(\mathrm{R}) \text {. }
$$

Eine strenge analytische Lösung der Differentialgleichung (9) für den kugelsymmetriscinen Fall konnte bis heute noch nicht gefunden werden. ${ }^{1}$ ) Die Gleichung läßt sich nur streng lösen²), wenn die Doppel-

1) Ein von Gyemant (loc. cit. S. 25) gegebener Ausdruck kann zwar als eine Näherung dienen, versagt aber gerade im wichtigsten Falle geringer Konzentrationen und mehrwertiger lonen.

2) Im ebenen Falle wird einfach

$$
\Delta \psi=\frac{\mathrm{d}^{2} \psi}{\mathrm{d} \cdot \mathrm{r}^{2}}=-\frac{4 \pi}{\mathrm{D}} \nu \text { e } \Sigma \mathrm{c}_{\mathrm{i}} z_{\mathrm{i}} \mathrm{e}^{-\mathrm{z}_{\mathrm{i}} \frac{\mathrm{e} \psi}{\mathrm{k} T}}
$$

Für den oben betrachteten Fall eines binären Elektrolyten mit zwei gleichwertigen Ionen von der Valenz $z$ wird

$\left.\begin{array}{l}c_{1}=c_{2}=c \\ z_{1}=-z_{2}=z\end{array}\right\}$

$$
\frac{\mathrm{d}^{2} \psi}{\mathrm{d} \mathbf{r}^{2}}=+\frac{8 \pi v \mathrm{ecz}}{\mathrm{D}} \sinh \mathrm{z} \frac{\mathrm{e} \psi}{\mathrm{kT}}
$$

Einmalige Integration liefert

$$
\frac{1}{2}\left(\frac{\mathrm{d} \psi}{\mathrm{dr}}\right)^{2}=\frac{8 \pi \mathrm{kT} \nu \mathrm{c}}{\mathrm{D}}\left[\operatorname{coshz} \frac{\mathrm{e} \psi}{\mathrm{kT}}-\mathrm{A}\right]
$$


schicht als eben betrachtet wird (d. h. daß man die Krümmung als klein gegen die Doppelschichtdicke annimmt). Diese Annahme ist aber wegen der für die kleinen Konzentrationen relativ großen Dicke nicht zulässig. Wir müssen daher den kugelsymmetrischen Fall näher studieren.

Für diesen wird (9)

$$
\Delta \psi=\frac{1}{\mathrm{r}^{2}} \frac{\mathrm{d}}{\mathrm{dr}}\left(\mathrm{r}^{2} \frac{\mathrm{d} \psi}{\mathrm{dr}}\right)=-\frac{4 \pi \nu}{\mathrm{D}}-\sum_{\mathrm{i}} \mathrm{c}_{\mathrm{i}} z_{\mathrm{i}} \mathrm{e}^{-\frac{z \mathrm{c} \psi}{\mathrm{k} T}} .
$$

Um die Lösung übersichtlicher zu gestalten, ist es zweckmäßigi) für $\psi$ und $\mathrm{r}$ neue Maßzahlen einzufühı一, indem man setzt:

$$
\left.\begin{array}{l}
\varphi=\frac{\mathrm{e}}{\mathrm{kT}} \cdot \psi=\frac{\psi}{\mathrm{A}}, \\
\eta=\frac{\mathrm{r}}{\sqrt{\frac{\mathrm{D} \mathrm{kT}}{4 \pi \mathrm{e}^{2} \nu \sum \mathrm{c}_{\mathrm{i}}}}}=\frac{\mathrm{r}}{\lambda},
\end{array}\right\}
$$

d. h. man mißt $\psi$ durch das Einheitspotential A ( $=25$ Millivolt) und $r$ durch eine charakteristische Länge $\lambda$ (in Debyes Theorie $\frac{1}{\mathrm{x}}$ ), die noch von den Konzentrationen abhängt:

$$
\left.\begin{array}{l}
\lambda=\sqrt{\frac{\mathrm{DkT}}{4 \pi \mathrm{e}^{2} v \Sigma \mathrm{c}_{\mathrm{i}}}}=\frac{\mu}{\sqrt{\Sigma \mathrm{c}_{\mathrm{i}}}} \\
\mu=\sqrt{\frac{\mathrm{DkT}}{4 \pi \mathrm{e}^{2} v}}=4,32 \cdot 10^{-5} \mathrm{~cm} .
\end{array}\right\}
$$

$\lambda$ bildet ein gewisses $\mathrm{MaB}$ für die Doppelschichtdicke.

Die erste Randbedingung liefert $\mathrm{A}=+1$. Somit

$$
\frac{\mathrm{d} \psi}{\mathrm{dr}}=-\sqrt{\frac{8 \pi \nu \mathrm{ck} T}{\mathrm{D}}}-2 \cdot \sinh \frac{z \mathrm{e} \psi}{2 \mathrm{kT}},
$$

woraus durch nochmalige Integration $\psi$ als Funktion des Abstandes erhalten wird. Für unsern $Z$ weck ist diese Integration nicht mehr nötig, denn die zweite Randbedingung liefert

$$
\begin{aligned}
& -\sigma=\frac{\mathrm{D}}{4 \pi}\left(\frac{\mathrm{d} \psi}{\mathrm{dr}}\right)_{\text {Obert. }}=-\sqrt{\frac{8 \pi \nu \mathrm{kTD}}{16 \pi^{2}}} \cdot 2 \sinh \frac{\mathrm{ze} \zeta}{2 \mathrm{kT}} \\
& \sigma=2 \sqrt{\frac{v \mathrm{kTD}}{2 \Pi}} \sqrt{\mathrm{c} \cdot \sinh } \frac{\mathrm{z}}{2} \frac{\mathrm{e}}{\mathrm{kT}} \xi=2 \alpha \sqrt{\mathrm{c} \sinh \frac{z}{2}} \frac{\xi}{\mathrm{A}},
\end{aligned}
$$

wo $A, \alpha$ die vorn gegebenen Werte haben. Durch Auflösung nach $\xi$ folgt (3):

$$
\xi=\frac{2 A}{z} \operatorname{arcsinh} \frac{\sigma}{2 \alpha \sqrt{c}} .
$$

1) P. Debye in seiner Vorlesung über "Ionen und Elektronen" an der Eidg. Techn. Hochschule Zürich W. S. 1922/23. 
Mit diesen neuen Maßzahlen erhält (12) die einfachere Form

$$
\frac{1}{\eta^{2}} \frac{\mathrm{d}}{\mathrm{d} \eta}\left(\eta^{2} \frac{\mathrm{d} \varphi}{\mathrm{d} \eta}\right)=-\frac{\sum_{\mathrm{i}} c_{\mathrm{i}} z_{\mathrm{i}} \mathrm{e}^{-z_{\mathrm{i}} \varphi}}{\sum_{\mathrm{i}} \mathrm{c}_{\mathrm{i}}}
$$

Debye und $\mathrm{Hückel}^{1}$ ) haben die Lösung dieser Differentialgleichung durchgefüht unter der Annahme, daß,

$$
\mathbf{z}_{\mathbf{i}} \varphi<<1 \text {. }
$$

Diese Voraussetzung ist für das elektrokinetische Potential gewöhnlich nicht erfüllt, denn die Messungen geben für $\xi$ Werte bis zu 100 Millivolt, also für $\varphi=\frac{\xi}{\mathrm{A}}$ Werte bis zu 4 .'.

Die Lösung von Debye und Hückel ist aber dennoch für uns von'großem Interesse, weil sie uns den Potentialabfall in einiger Ent. fernung von der Teilchenoberfläche gibt, wo das Potential so klein ist, da $\grave{B}$ es die Bedingung (15) erfüllt. In diesem Bereiche der Doppelschicht kann man dann setzen:

und erhält aus (12)

$$
\mathrm{e}^{-z_{\mathrm{i}} \varphi}=1-z_{\mathrm{i}} \varphi
$$

$$
\frac{1}{\eta^{2}} \frac{\mathrm{d}}{\mathrm{d} \eta}\left(\eta^{2} \frac{\mathrm{d} \varphi}{\mathrm{d} \eta}\right)=\frac{\sum \mathrm{c}_{\mathrm{i}} \mathrm{z}_{\mathrm{i}}}{\sum \mathrm{c}_{\mathrm{i}}}+\frac{\sum \mathrm{c}_{\mathrm{i}} \mathrm{z}_{\mathrm{i}}^{2}}{\sum \mathrm{c}_{\mathrm{i}}} \cdot \varphi .
$$

Der erste Summand verschwindet, da $\sum c_{\mathbf{i}} z_{\mathbf{i}}$ die Gesamtladung aller Ionen im Elektrolyten darstellt, die aber stets Null ist.

Die allgemeine Lösung der Gleichung

$$
\begin{aligned}
& \left.\begin{array}{rl}
\frac{1}{\eta^{2}} \frac{\mathrm{d}}{\mathrm{d} \eta}\left(\eta^{2} \frac{\mathrm{d} \varphi}{\mathrm{d} \eta}\right) & =\mathrm{x}^{2} \varphi \\
\therefore & \mathrm{x}^{2}=\frac{\sum \mathrm{c}_{\mathrm{i}} \mathrm{z}_{\mathrm{i}}^{2}}{\sum \dot{c}_{\mathrm{i}}}
\end{array}\right\} \\
& \varphi=\mathrm{K} \cdot \frac{\mathrm{e}^{-x \eta}}{\eta}+\mathrm{K}_{1} \frac{\mathrm{e}^{+x \eta}}{\eta}
\end{aligned}
$$

Die erste Randbedingung. $(10 \mathrm{a})$ verlangt $\mathrm{K}_{1}=0$; somit

$$
\varphi=\mathrm{K} \frac{\mathrm{e}^{-\mathrm{x} \eta}}{\eta}
$$

Diese Formel gibt uns also, bis auf eine multiplikative Konstante genau, den Potentialabfall im Bereiche, wo/für alle auftretenden Wertigkeiten $\mathrm{z}_{\mathrm{i}} \varphi<<1$.

1) P. Debye und E. Hückel, Physik. Zeitschr. 24; 185/206 (1923). 
Die Konstante K. ergibt sich aus der zweiten Randbedingung (10b). Da aber die Lösung (17) für $r=R$ im allgemeinen nicht mehr genúgend genau ist, so bleibt $\mathrm{K}$ unbestimmt. Nur im Grenzfalle kleiner $\xi$ kann man nach (10b) setzen:

woraus

$$
\left(\frac{\mathrm{d} \psi}{\mathrm{d} r}\right)_{\mathrm{r}=\mathrm{R}}=\frac{\mathrm{A}}{\lambda}\left(\frac{\mathrm{d} \Phi}{\mathrm{d} \eta}\right)_{\eta_{0}=\frac{\mathrm{R}}{\lambda}}=-\frac{4 \pi \sigma}{\mathrm{D}_{\mathrm{s}}}
$$

Da $\varphi_{\mathbf{0}}=\frac{\psi_{\mathbf{0}}}{\mathrm{A}}=\frac{\xi}{\mathrm{A}}$ hat man dann die gesuchte Beziehung zwischen $\xi, \sigma$ und den Konzentrationen $c_{i}$

$$
\xi=\frac{4 \pi \lambda}{\mathrm{D}} \frac{\eta_{0}}{\eta_{0} \mathrm{x}+1} \sigma
$$

worin $\eta_{0}=\frac{R}{\lambda}$, während $\lambda, x$ durch (14) resp. (16) aus den Konzen. trationen bestimmt sind.

Im allgemeinen ist aber die Debyesche Lösung (18) in der Nähe der Teilchenoberfläche nicht mehr anwendbar ${ }^{1}$ ); wir müssen daher dort eine strengere Lösung der Differentialgleichung (12') suchen. Dies könnte beispielsweise versucht werden durch Berücksichtigung eines weiteren Terms in der Taylorentwicklung von $e^{-z_{i} \varphi}$. Wir wollen aber hier eine graphische Methode entwickeln, die das Problem mit genügender Genauigkeit löst.

Aus jeder Differentialgleichung zweiter Ordnung kann ja, wenn in einem Punkte $\eta_{0}$ der Funktionswert $\varphi_{0}$-und die Ableitung $\varphi_{0}^{\prime}$ gegeben sind, . die zweite Ableitung $\varphi_{, 0}^{\prime \prime}$ in diesem Punkte berechnet werden. Aus diesen Daten läßt sich der Funktionswert und die Tangente in einem benachbarten Punkte $\eta_{0}+\Delta \eta$ mit beliebiger Genauig. keit berechnen, wenn nur der Schritt $\Delta \eta$ nicht zu groß gewählt wird. Durch sukzessive Anwendung dieses Verfahrens kann die Integrationskurve schrittweise gefunden werden. Wir müssen nur in einem Ausgangspunkt $\eta_{0}$ den Funktionswert und die Ableitung kennen. Ein solcher Ausgangspunkt wird uns durch die Debyesche Näherung mit hinreichender Genauigkeit gegeben, wenn wir nur für $\eta_{0}$ einen Wert wählen, wo $p_{0}$ so klein ist, daß die Bedingung (15) hinreichend stark erfüllt ist.

Ein solches Verfahren erscheint auf den ersten Blick sehr umständlich. Es läßt sich aber in unserem Falle sehr einfach gestalten,

1) Auch in Debyes Theorie der Elektrolyte ist für hochwertige lonen die Bedingung (15) nicht mehr erfüllt. Die Anwendung des hier entwickelten Verfahrens kann daher auch eine kleirie Korrektur der Elektrolyttheorie ergeben. Siehe H. Müller, Phys. Zeitschr. 28, 324 (1927) 
wenn man an Stelle der Variabeln $\eta$ eine andere einführt durch die Substitution:

$$
\mathrm{y}=\frac{1}{\eta}
$$

Diese Transformation bietet zwei Vorteile:

1. An Hand der Debeyeschen Näherung kann man leicht zeigen, daß der größte und wichtigste Teil des Potentialabfalls det diffusen Doppelschicht sich in einem sehr kleinen Intervall der $\eta$-Achse vollzieht, das zwischen $\eta=0$ und $\eta=1$ liegt. Durch die Transformation wird dieses Gebiet auseinandergezogen und der steile und stark gekrümmte $\varphi(\eta)$-Abfall erscheint in der $\varphi, \mathrm{y}$-Ebene als eine glatte, schwach gekrümmte Kurve, für die das graphische Verfahren eine sehr gute Annäherungsmethode sein muß.

2. Durch die Transformation erhält die Differentialgleichung (12') eine sehr einfache und für die Rechnung höchst praktische Form:

$$
\frac{d^{2} \varphi}{d y^{2}}=-\frac{1}{y^{4}} \frac{\sum_{i} c_{i} z_{i} e^{-z_{1} \varphi}}{\sum_{i} c_{i}} .
$$

Die Randbedingungen (10) lauten mit diesen Variabeln für

$$
\mathrm{y}=0 \quad \varphi=0 \quad \frac{\mathrm{d} \varphi}{\mathrm{dy}}=\varphi^{\prime}=0
$$

und für

$$
\begin{gathered}
\mathrm{y}=\mathrm{y}_{0}=\frac{1}{\eta_{0}}=\frac{\lambda}{\mathrm{R}}, \\
\sigma=-\frac{\mathrm{DA}}{4 \pi \lambda} \mathrm{y}_{0}^{2} \varphi_{0}^{\prime}, \quad \text { wo } \varphi_{0}^{\prime}=\left(\frac{\mathrm{d} \varphi}{\mathrm{dy}}\right)_{\mathrm{y}=\mathrm{y}_{0}}
\end{gathered}
$$

während die der Debyeschen Lösung entsprechende Näherung lautet:

$$
\varphi=\mathrm{Kye}^{-\frac{\dot{x}}{y}}
$$

Diese Näherung ist gut für kleines y, wo $\varphi$ die Bedingung (15) erfüllt. Sie erfüllt natürlich die erste Randbedingung (21a).

Um nun irgendeine Lösung der Differentialgleichung (20) zu konstruieren, die mindestens die erste Randbedingung erfüllt, geht man von der Näherung (22) aus, indem man $\mathrm{K}$ einen beliebigen Wert von dem Problem angepaßter Größenordnung ( $z$ wischen 1 und 10) erteilt. Im Gebiete, wo diese Näherung sicher noch sehr gut sein muß, fassen wir einen Punkt $\mathrm{y}_{0}$ heraus und benutzen ihn und die durch die Näherung bestimmten Werte von $\varphi_{0}$ und $\varphi_{0}^{\prime}$ als Ausgangspunkt der schrittweisen Integration der ungekürzten Differentialgleichung (20). Diese liefert dann für diesen Punkt

$$
\varphi_{0}^{\prime \prime}=-\frac{1}{y_{0}^{4}} \frac{\sum c_{i_{i}} z_{i} e^{-z_{j} \varphi_{0}}}{\sum c_{i}} .
$$


Aus $y_{0}, \varphi_{0}, \varphi^{\prime}{ }_{0}$ und $\varphi^{\prime \prime}{ }_{0}$ läßt sich Funktionswert und Tangente in einem benachbarten Punkte $\varphi_{0}+\Delta \mathrm{y}$ bestimmen:

$$
\begin{aligned}
& \varphi_{y_{0}+\Delta y}^{\prime}=\varphi_{y_{0}}^{\prime}+\Delta y \cdot \overline{\varphi_{0}^{\prime \prime}} \\
& \varphi_{y_{0}+\Delta y}=\varphi_{0}+\Delta y \overline{\varphi_{0}^{\prime}}
\end{aligned}
$$

und daraus wiederum nach $(20)$ :

$$
\varphi_{y_{0}+\Delta y}^{\prime \prime}=-\frac{1}{\left(y_{0}+\Delta y\right)^{4}} \frac{\sum c_{i} z_{i} e^{-z_{i} \varphi_{y_{0}}+\Delta y}}{\sum c_{i}} .
$$

Die überstrichener. Größen werden nach dem Mittelwertsatz der Differentialgleichung korrigiert. Der Schritt $\Delta \mathrm{y}$ wird nun so klein gewählt, daß diese letzteren Korrektionen unbedeutend werden. Indem man dieses Verfahren mit den neugefundenen Punkten sukzessive wiederholt, kann man die Kurve beliebig weit extrapolieren. Da es sich zeigt, daß im allgemeinen die Krümmung dieser Kurven sehr gering ist, genügen 20 bis 30 Schritte, um die nötige Kurve zu berechnen. Sie weichen von der Debyeschen Näherung ziemlich stark ab bei hohen Potentialen und mehrwertigen Ionen. Bei kleinen Potentialen fallen sie mit der Näherung zusammen und bestätigen damit die Güte derselben.

Indem man die Konstante $\mathrm{K}$ variiert, kann man auf diese Weise ein ganzes System von Integrationskurven $\varphi(\mathrm{y})$ und die zugehörigen $\varphi^{\prime}(\mathrm{y})$ konstruieren, die alle die ersten Randbedingungen erfüllen. Man muß nur unter diesen Kurven diejenige heraussuchen, welche auch die zweite Randbedingung (21b) erfüllt. Ist die spezifische Teilchenladung $\sigma$, der Teilchenradius und die Konzentrationen gegeben, so ist $\mathrm{y}_{\mathbf{0}}=\frac{2}{\mathrm{R}}$ bestimmt, und wir haben nur diejenige Integrationskurve zu suchen, die für diesen $y_{0}$-Wert den aus $(21 \mathrm{~b}$ ) bestimmten Wert von $\varphi_{0}^{\prime}$ hat. Dies wird durch Interpolation der berechneten Kurven immer leicht möglich sein. Der Wert $\boldsymbol{\varphi}_{0}$ dieser Kurve liefert dann das gesuchte elektrokinetische Potential $\xi=$ A $_{0}$.

Tatsächlich interessiert uns aber zum Beweise der unter I entwickelten Hypothese nicht diese Aufgabe, denn wir kennen ja die Teilchenladung $\sigma$ nicht, sondern wollen gerade diese aus den aus dem Experiment bekannten Werten $c_{i}$ und $\xi$ bestimmen. Nehmen wir noch den Teilchenradius $R$ als bekannt an, so fragen wir: Welche Ladung müßte nach unserer Theorie das Teilchen haben, um bei gegebenen Konzentrationen $c_{i}$ das gemessene Potential $\xi$ zu zeigen? Wir kennen somit $\varphi=\frac{\xi}{\mathrm{A}}$ und $\mathrm{y}=\frac{\lambda}{\mathrm{R}}$ und haben durch Interpolation die Inte- 
grationskurve durch diesen Punkt $\varphi$, y und ihre Ableitung in diesem Punkt zu bestimmen. Dann liefert wieder (21b) den gesuchten Wert von $\sigma$.

Indem wir also für verschiedene Elektrolyte das System der Integrationskurven bestimmen und zu gemessenen $(\xi, c)$ Werten die entsprechenden Werte der Teilchenladung $\sigma$ berechnen, können wir die Änderung dieser Ladung mit der Elektrolytkonzentration, d. h. die Adsorption (in unserer erweiterten Definition) vollkommen verfolgen. Zur Durchführung dieser Rechnung ist neben genau gemessenen $\xi$ (c)Kurven nur die Kenntnis des Teilchenradius nötig. Speziell können wir damit zeigen, wieviel der Potentialerniedrigung der 'Doppelschichtsänderung und wieviel der Adsorptionsänderung zugeschrieben werden muß.

Voraussetzung bei der ganzen Theorie ist nur, daß die Ionen außerhalb der Abreißschicht eine diffuse Doppelschicht bilden und daß die Ionen als Punkte betrachtet werden können (keine Hydratation und kleine Konzentrationen).

III.

Wir wollen nun dieses Verfahren speziell für die Messungen von Freundlich und $Z \mathrm{eh}^{1}$ ) durchführen, um unsere Behauptung zu belegen, daß die Doppelschichtsänderung der wesentlichste Effekt zum Verständnis der $\xi$ (c)-Kurven ist. In der früher erwähnten Messung von Fried und $\mathrm{Pauli}{ }^{\circ}$ ) sind leider nur drei Punkte der $\xi(\mathrm{c})$-Kurve bestimmt, so daß dort die Extrapolation etwas gewagt ist.

Die experimentelle Prüfung der Theorie begegnet einer Reihe von Schwierigkeiten teils experimenteller, teils theoretischer Natur. Eine Pıüfung der Theorie verlangt vor allem eine genaue Kenntnis des Absolutwertes des Potentials $\xi$. Dieses wird nach der von $\mathrm{H}$ ückel gegebenen Formel (1) aus der kataphoretischen Wanderungsgeschwindigkeit $u$ bestimmt. Die Bestimmung von $u$, die gewöhnlich nach makroskopischen Methoden von $\mathrm{Burton}^{3}$ ) geschieht, hat aber eine Reihe von schwer zu eliminierenden Fehlerquellen, so daß genaue reproduzierbare Messungen recht schwierig sind.

Die Anwendung der Formel von Hückel setzt wesentlich voraus, daß das Teilchen kugelförmig ist und daß der Potentialabfall in der Doppelschicht Kugelsymmetrie hat. Die Bedingung des kugelsym-

1) H. Freundlich u. P. Zeh, Zeitschr. f. phys. Chem. 114, 65 (1925).

3) Fried u. Pauli, Koll.-Zeitschr. 36, 138 (1925).

3) Burton, Phil. Mag. 11, 440 (1906'. Einen zusammenfassenden Bericht uber die Genauigkeiten und Schwierigkeiten dieser und anderer Methoden bei Kruyt: Koll.Zeitschr Dezember 1925 
metrischen Potentials um ein kugelförmiges Teilchen dürfte trotz der relativ beträchtlichen Dicke der Doppelschicht erfüllt sein, wenn nur die Wanderungsgeschwindigkeit keine zu rasche ist. Ob die Teilchen Kugelform haben oder nicht, kann mit optischen Methoden einigermaßen geprüft werden, bleibt aber stets etwas unsicher. Da Deby $e^{1}$ ) gezeigt hat, daß der Zahlenfaktor, der für die Kugel $6 \pi$ ist, sich mit der Teilchenform ändert, bleibt daher der Absolutwert von $\xi$ stets etwas unsicher.

Auch die genaue Angabe der Konzentrationen $c_{\mathfrak{i}}$ aller Ionen im Kolloid ist sehr schwierig, da es sich um sehr geringe Mengen von wenigen, oft nur Bruchteilen von Mikromolen je Liter handelt. Man weiß nun aus den Versuchen Lottermosers ${ }^{2}$ ), daß für die Existenzmöglichkeit eines Kolloides stets eine minimale Elektrolytkonzentra. tion nötig ist. Abgesehen von den $\mathrm{H}^{+}$- und $\mathrm{OH}^{-}$-Ionenkonzentrationen des Wassers haben wir stets eine geringe Elektrolytkonzentration von etwa 1 Mikromol, die aus dem Bildungsprozeß des Kolloides herstammt und die durch Dialyse nie völlig entfernt werden kann. Diese ursprüngliche Konzentration wird gewöhnlich nicht berücksichtigt; sie mag aber bei mehrwertigen Ionen, wo die $\xi$ (c)-Kurve für Konzentrationen kleiner als 1 Mikromol stark abfällt, eine wesentliche Rolle spielen. Auch durch die Adsorption können die c-Werte gefälscht werden, indem die in die Theorie eingehenden Kंonzentrationen sich von der dem Kolloid beigemischten unterscheidet um den Teil, der an den Micellen adsorbiert wird. In schwach konzentrierten Kolloiden mag dieser Einfluß klein sein; in stärker konzentrierten spielt er sicher eine Rolle, wie der Einfluß der Konzentration und der Micellengröße der Kolloide auf die Koagulationskonzentration zeigt.

Wir müssen hier von all diesen Unbestimmtheiten absehen und wollen die gegebenen $\xi(c)$-Kurven als ideal richtig annehmen. Wir bemerken nur, daß eventuelle Abweichungen von der Theorie bei ganz geringen Elektrolytkonzentrationen vielleicht auf diese Unbestimmtheiten zurückzuführen sind.

Auf die Diskussion des Einflusses der Teilchenradien R, den wir auch kennen müssen und der auch nur in gewissen Grenzen bekannt ist, kommen wir später zurück.

Die Freundlichschen Messungen sind durchgeführt an einem

1) P. Debye u. E. Hückel, Phys. Zeitscbr. 25, 49 (1924).

2) Lottermoser, Zeitschr. f. phys. Chem. 60, 451 (1907). Gerade diese Versuche beweisen, daß die Existenz eines Kolloides an die Existenz einer Doppelschicht gebunden ist. 
negativen $\mathrm{As}_{2} \mathrm{~S}_{3}-$ Sol. $^{1}$ ) Die Voraussetzung der Kugelgestalt der Micellen dürfte bei diesem Sol erfüllt sein. Als Elektrolyt wurden Salze benutzt von der Form $A_{1}^{(+z)} B_{z}^{(-1)}$ wo $A^{+z}$, das ,entladende" positive Ion, ein $z$-wertiges Komplexion ist; das negative einwertige Ion war $\mathrm{Cl}^{-}$- Das $\xi$-Potential ist dementsprechend negativ. Um die Vorzeichenfrage einfacher zu gestalten, wollen wir das Sol positiv betrachten und ersetzen in Gedanken den Elektrolyten durch ein Salz $\mathrm{A}_{1}^{(-z)} \mathrm{B}_{z}^{(+1)}$. Die Verhält. nisse bleiben dann genau dieselben, nur sind jetzt $\xi$ und $\sigma$ positiv.

Für einen solchen binären Elektrolyten haben wir also, wenn c die Konzentration des Elektrolyten ist:

$$
\begin{array}{ll}
c_{1}=c & z_{1}=-z, \\
c_{2}=z c & z_{2}=+1 .
\end{array}
$$

Die Differentialgleichung (20) wird somit

$$
\frac{\mathrm{d}^{2} \varphi}{\mathrm{d} \mathrm{y}^{2}}=\frac{z}{(z+1)} \frac{1}{\mathrm{y}^{4}}\left(\mathrm{e}^{\mathrm{z} \varphi}-\mathrm{e}^{-z \varphi}\right) .
$$

In der korrespondierenden Näherung ist nach (16)
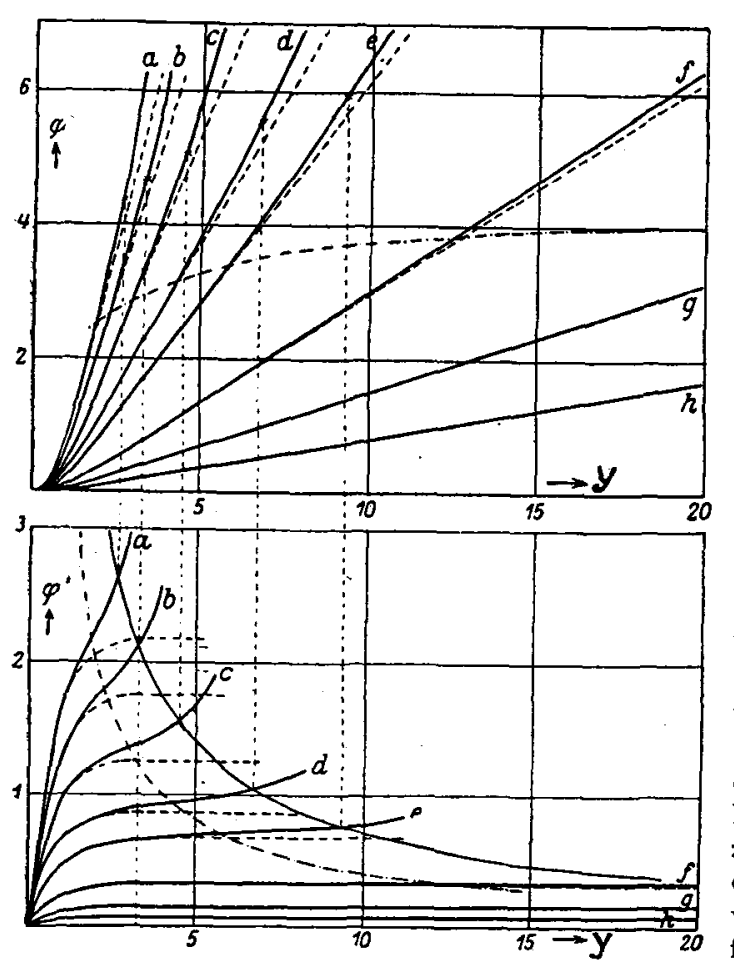

Fig. 1.

$\varphi(\mathrm{y})$ und $\varphi^{\prime}(\mathrm{y})$ für $1: 1$ wertige Elektrolyte.

$$
\mathrm{x}^{2}=\frac{\mathrm{cz^{2 } + 2 \mathrm { c }}}{\mathrm{cz}+\mathrm{c}}=z
$$

somit lautet die Näherung (22)

$$
\varphi=\mathrm{Ky} \mathrm{e}^{-\frac{\sqrt{\mathrm{z}}}{\mathrm{y}}}
$$

Die Tabellen 1, 2, 3,4 geben einige nach dem in II besprochenen Verfahren berechnete Integrationskurven für $z=1,2,3,4$. Die Kurven sind in den Figuren 1, 2, 3, 4 dargestellt. Die Ausgangswerte $y_{0}, \varphi_{0}$ und die Größe der Schritte

1) Die Messungen an dem positiven $\mathrm{Fe}_{2} \mathrm{O}_{3}$-Sol können wir nicht in Betracht . ziehen, weil dort die Teilchenform nicht sicher steht, wahrscheinlich nicht kugel. förmig ist. Bei den Messungen mit $\mathrm{KCl}$ spielen Hydratationseinflüsse eine Rolle. 
sind überall so klein gewählt, daß die Zahlenwerte auf mindestens die dritte Dezimale genau sind, also die Meßgenauigkeit der $\xi$ (c)-Kurven überschreiten.

In den Figuren sind neben unseren Kurven auch diejenigen gestrichelt eingetragen, die sich nach der Näherung von Debye und Hückel ergeben. Man sieht, daß für große Potentialwerte die Korrekturen zur Debyeschen Formel recht bedeutend werden. Das kommt vor allem stark zum Ausdruck, wenn man in Fig. 1 die beiden Systeme der Kurven vergleicht. (Fig. 1 stammt aus meiner Arbeit: Die Aktivität kleiner Ionen, Physik. Zeitschr., in welcher auch die Frage diskutiert ist, bis zu welchen Potentialen die Debyesche Näherung angewen det werden darf.)

Aus diesen Kurven können wir nun nach der vorn ge-

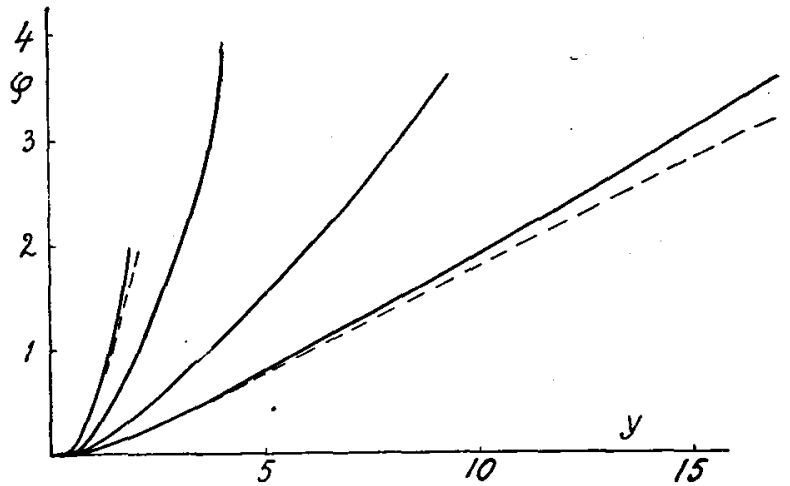

Fig. 2. $\varphi(y)$ für $2: 1$ wertige Elektrolyte.

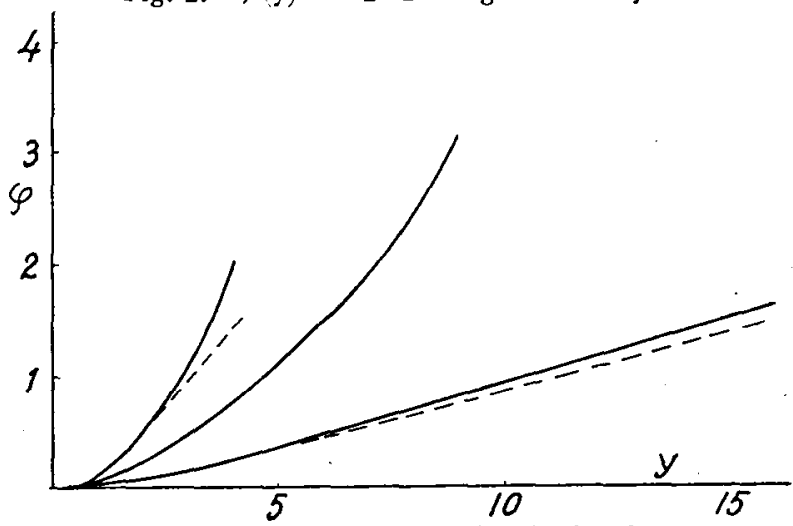

Fig. 3. $\varphi(\mathrm{y})$ für $3: 1$ wertige Elektrolyte.

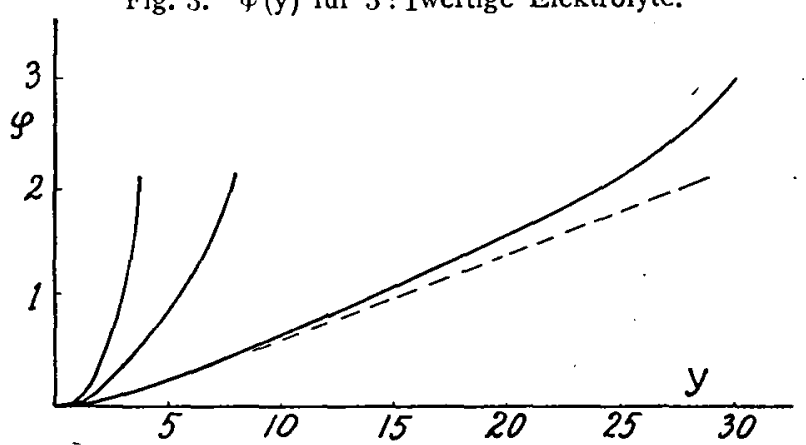

Fig. 4. $\varphi(y)$ für $4: 1$ wertige Elektrolyte. gebenen Methode aus den $\xi$ (c)-Kurven von Freundlich und $Z$ eh auf die Adsorptionskurven $\boldsymbol{\sigma}(\mathrm{c})$ schließen. Wir haben zu diesem $Z_{\text {wecke, }}$ um den Einfluß der Meßfehler zu umgehen, nicht direkt gegebene Werte 
Tabelle 1.

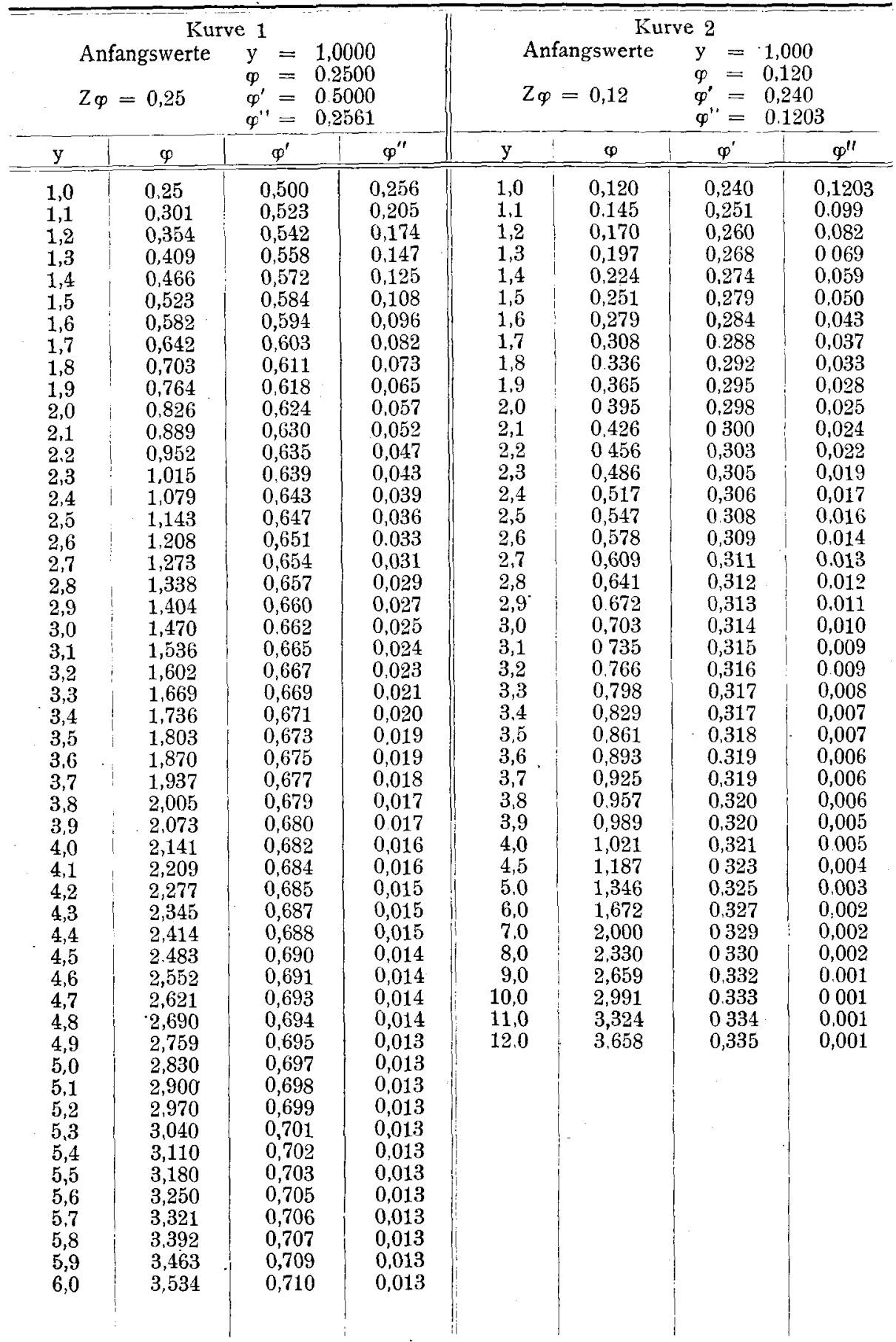


Für 1-1-Wertigkeit.

\begin{tabular}{|c|c|c|c|c|c|c|c|c|c|c|}
\hline & Kur & e 3 & & & & & & & & \\
\hline$Z \varphi$ & 0,2 & $\begin{array}{l}y= \\
\varphi= \\
\varphi^{\prime}= \\
\varphi^{\prime \prime}=\end{array}$ & $\begin{array}{l}.800 \\
.200 \\
, 574 \\
.492\end{array}$ & $\begin{array}{l}\text { Anf } \\
Z_{\varphi}\end{array}$ & 0,2 & $\begin{array}{l}y= \\
\varphi= \\
\varphi^{\prime}= \\
\varphi^{\prime \prime}=\end{array}$ & & $Z_{\varphi}=$ & 0,5 & $\begin{array}{l}=0,800 \\
=0,500 \\
=1,437 \\
=1,27\end{array}$ \\
\hline$y$ & $\varphi$ & $\varphi^{\prime}$ & $\Phi^{\prime \prime}$ & $\mathrm{y}$ & $\varphi$ & $\varphi^{\prime}$ & $\varphi^{\prime \prime}$ & $\mathrm{y}$ & $\varphi$ & $\Phi^{\prime}$ \\
\hline 0.80 & 0,200 & 0,574 & 0,492 & 0.60 & 0.200 & 0,890 & 1,55 & 0.80 & 0,500 & 1,437 \\
\hline 0,85 & 0,229 & 0,598 & 0,44 & 0.62 & 0,218 & 0920 & 1,48 & 0,82 & 0,529 & 1,462 \\
\hline 0,90 & 0,260 & 0,630 & 0.40 & 0,64 & 0237 & 0,950 & 1,42 & 0.84 & 0,558 & 1,486 \\
\hline 0.95 & 0,292 & 0,649 & 0,36 & 0.66 & 0.256 & 0,977 & 1,37 & 0.86 & 0,588 & 1,510 \\
\hline 1,00 & 0,326 & 0,667 & 0.33 & 0,68 & 0,276 & 1,004 & 1,32 & 0,88 & 0,619 & 1.532 \\
\hline 1,05 & 0,360 & 0,683 & 030 & 0,70 & 0,296 & 1,030 & 1,25 & 0,90 & 0,649 & 1,554 \\
\hline 1,10 & 0,395 & 0,697 & 0,27 & 0,72 & 0.317 & 1,054 & 1,20 & 0,92 & 0,681 & 1.575 \\
\hline 1,15 & 0,429 & 0.710 & 025 & 0,74 & 0,338 & 1.078 & 1,15 & 0,94 & 0,712 & 1.595 \\
\hline 1,20 & 0,466 & 0,723 & 0,23 & 0,76 & 0.360 & 1,101 & 1,10 & 0.96 & 0.744 & 1,615 \\
\hline 1,25 & 0,503 & 0,734 & 0,21 & 0,78 & 0,382 & 1,122 & 1,05 & 0,98 & 0.777 & 1,634 \\
\hline 1.30 & 0,540 & 0,744 & 0,20 & 0,80 & 0,405 & 1,142 & 1,01 & 100 & 0.810 & 1,652 \\
\hline 1,35 & 0,577 & 9.754 & 0,18 & 0,82 & 0,428 & 1,163 & 1,00 & 1,05 & 0894 & 1,696 \\
\hline 1,40 & 0,615 & 0.763 & 0,17 & 0.84 & 0,451 & 1,182 & 0,96 & 1,10 & 0,980 & 1,736 \\
\hline 1,45 & 0,653 & 0.771 & 0,16 & 0.86 & 0,475 & 1,200 & 0,92 & 1,15 & 1,067 & 1,774 \\
\hline 1,50 & 0,692 & 0.779 & 0,15 & 0.88 & 0.499 & 1,218 & 0,89 & 1,20 & 1,157 & 1,810 \\
\hline 1,55 & 0,731 & 0.787 & 0,14 & 0.90 & 0,523 & 1,235 & 0,85 & 1,25 & 1,248 & 1,844 \\
\hline 1,60 & 0,771 & 0,794 & 0,13 & 0,92 & 0.548 & 1,251 & 0,82 & 1.30 & 1,341 & 1,876 \\
\hline 1,65 & 0,811 & 0.800 & 0,12 & 0,94 & 0,574 & 1,267 & 0,79 & 1,35 & 1,436 & 1,906 \\
\hline 1,70 & 0,851 & 0,806 & 0,12 & 0,96 & 0,599 & 1,282 & 0,77 & 1,40 & 1,532 & 1,935 \\
\hline 1.75 & 0,891 & 0,812 & 0. & 0.98 & 0.625 & 1,297 & 0,73 & 1,45 & 1,629 & 1,964 \\
\hline 1.80 & 0,932 & 0,817 & 0,10 & 1,00 & 0.651 & 1,311 & 0.71 & 1,50 & 1,739 & 1,991 \\
\hline 1,85 & 0.973 & 0,822 & 0,10 & 1,05 & 0.717 & 1,345 & 0,67 & 1,55 & 1,829 & 2,018 \\
\hline 1,90 & 1,014 & 0,827 & 0.097 & 1,10 & 0.785 & 1,376 & 0,62 & 1,60 & 1,931 & 2,044 \\
\hline 1.95 & 1,055 & 0,831 & 0,092 & 1,15 & 0.855 & 1,404 & 0,57 & 1,65 & 2,033 & 2,070 \\
\hline 2,00 & 1,097 & 0,836 & 0,088 & 1,20 & 0.926 & 1,431 & 0,53 & 1,70 & 2.138 & 2,095 \\
\hline 2,1 & 1,181 & 0.814 & 0.083 & 1,25 & 0.998 & 1,456 & $0, \breve{50}$ & 1.75 & 2.243 & 2,120 \\
\hline 2,2 & 1,266 & 0,851 & 0.076 & 1,30 & 1,071 & 1,480 & 0,47 & 1,80 & 2,350 & 2,145 \\
\hline 2,3 & 1,351 & 0,858 & 0,070 & 1,35 & 1,146 & 1,501 & 0,44 & 1,85 & 2,457 & 2,169 \\
\hline 2,4 & 1,437 & 0864 & 0,065 & 1.40 & 1.221 & 1,522 & 0,41 & 1,90 & 2.566 & 2,194 \\
\hline 2,5 & 1,523 & 0,870 & 0,060 & 1,45 & 1,298 & 1,541 & 039 & 1,95 & 2.676 & 2,219 \\
\hline 2,6 & 1,611 & 0,875 & 0,056 & 1,50 & 1.375 & 1.560 & 0,37 & 2,00 & & 2,244 \\
\hline 2,7 & 1.698 & 0,880 & 0,052 & 1,055 & 1,454 & 1,578 & 0,36 & 2,05 & 2,901 & 2,270 \\
\hline 2,8 & 1,787 & 0,885 & 0,050 & 1,60 & 1,533 & 1,595 & 0,33 & 2,10 & 3,015 & 2.296 \\
\hline 2,9 & 1,875 & 0,890 & 0,049 & 1.65 & 1,614 & 1,611 & 0,32 & 2,15 & 3,1 & 2.322 \\
\hline 3,0 & 1,964 & 0,894 & 0,044 & 1,70 & $1,69 \mathbf{a}$ & 1,627 & 0,31 & 2,20 & & 2,349 \\
\hline 3,1 & 2,054 & 0,899 & 00 & 1,75 & 1,776 & 1,642 & 0,30 & 2,25 & & 2,377 \\
\hline 3,2 & 2,144 & 0,903 & 0,041 & 1,80 & 1,859 & 1,658 & 0,30 & 2,30 & & 2,405 \\
\hline 3,3 & 2,234 & 0,907 & 0040 & 1,85 & 1,942 & 1,672 & 0,29 & 2,35 & 3,605 & $\begin{array}{l}2,400 \\
2,435\end{array}$ \\
\hline 3.4 & 2,325 & 0,911 & 0,039 & 1,90 & 2,026 & 1.687 & 0,28 & & & \\
\hline 3.5 & 2,416 & 0.914 & 0,038 & 1,95 & 2,111 & 1,701 & 0,28 & & & \\
\hline 3,6 & 2,508 & 0,918 & 0,037 & 2,00 & 2,196 & 1,715 & 0,28 & & & \\
\hline & 2,600 & 0,922 & & & 2,369 & 1,7 & 0,28 & & & \\
\hline 3,8 & 2.692 & 0925 & 0.0 & 2,20 & 2,544 & 1,770 & 0,27 & & & • \\
\hline 3,9 & 2,785 & 0,929 & 0,035 & 2,30 & 2,723 & 1,797 & 0,27 & & & \\
\hline 4,0 & 2,878 & 0,932 & 0.035 & 2,40 & 2.904 & 1,821 & 0,27 & & & \\
\hline 4,1 & 2,971 & 0,936 & 0,034 & 2,50 & 3,088 & 1,851 & 0,28 & & & \\
\hline 4,2 & 3.065 & 0.940 & 0,0 & 2.60 & 3,274 & 1,880 & 0,29 & & & \\
\hline 4,3 & 3,159 & 0,943 & 0.0 & 2.70 & 3.461 & 1,909 & 0,30 & & & \\
\hline 4,4 & 3.253 & 0.946 & 0,034 & 2,80 & 3,656 & 1,940 & 0,31 & & & \\
\hline 4,5 & 3,348 & 0,950 & 0.035 & & & & & & & \\
\hline 4,6 & 3.443 & & & & & & & & & \\
\hline 4,7 & 3.538 & 0,957 & 0,035 & & & & & & & \\
\hline 48 & 3,634 & 0.961 & 0,036 & & & & & & & \\
\hline & & & & & & & & & & \\
\hline
\end{tabular}


Tabelle $2^{1}$ ).

\begin{tabular}{|c|c|c|c|c|c|c|c|c|c|}
\hline \multicolumn{5}{|c|}{ Kurve 1} & \multicolumn{5}{|c|}{ Kurve 2} \\
\hline \multirow{3}{*}{\multicolumn{5}{|c|}{$\begin{aligned} \text { Anfangswerte } y & =1,000 \\
& \varphi=0,050 \\
Z_{\varphi}=0,10 \quad \varphi^{\prime} & =0,173 \\
\varphi^{\prime \prime} & =0,250\end{aligned}$}} & \multirow{3}{*}{\multicolumn{5}{|c|}{ 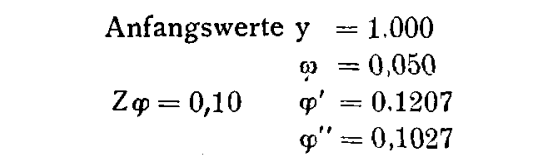 }} \\
\hline & & & & & & & & & \\
\hline & & & & & & & & & \\
\hline & & & & & & & & & \\
\hline$\Delta y$ & $\mathrm{y}$ & $\varphi$ & $\varphi^{\prime}$ & $\varphi^{\prime \prime}$ & $\Delta \mathrm{y}$ & $y$ & $\varphi$ & $\varphi^{\prime}$ & $\varphi^{\prime \prime}$ \\
\hline \multirow[t]{6}{*}{$(0,05)$} & 0,8 & 0,050 & 0,173 & 0,250 & $(0,1)$ & 1,0 & 0,050 & 0,121 & 0,102 \\
\hline & 1,0 & 0,089 & 0,216 & 0,185 & & 1,5 & 0,121 & 0,158 & 0,051 \\
\hline & 1.2 & 0,136 & 0,249 & 0,141 & & 2,0 & 0,205 & 0,177 & 0,029 \\
\hline & 1,4 & 0,198 & 0.274 & 0.116 & & 2,5 & 0,297 & 0.189 & 0,018 \\
\hline & 1,6 & 0,255 & 0,296 & 0,091 & & 3,0 & 0,394 & 0,196 & 0,012 \\
\hline & 1,8 & 0,316 & 0.312 & 0,074 & & 3,5 & 0,493 & 0202 & 0,009 \\
\hline \multirow[t]{5}{*}{$(0,1)$} & 2,0 & 0380 & 0,326 & 0,061 & & 4,0 & 0,595 & 0,206 & 0,007 \\
\hline & 2,2 & 0.446 & 0,337 & 0.051 & $(0,2)$ & 4,2 & 0,636 & 0,207 & 0,006 \\
\hline & 2,4 & 0,515 & 0,347 & 0,046 & & 4,4 & 0,678 & 0,208 & 0,006 \\
\hline & 2,6 & 0,585 & 0,355 & 0,039 & & 4,6 & 0,720 & 0,210 & 0,005 \\
\hline & 2,8 & 0,657 & 0,363 & 0.035 & & 4,8 & 0.762 & 0.211 & 0,005 \\
\hline \multirow[t]{16}{*}{$(0,2)$} & 3,0 & 0,730 & 0,369 & 0,031 & & 5,0 & 0.804 & 0,212 & 0,005 \\
\hline & 3,2 & 0804 & 0.375 & 0030 & & 5,3 & 0,846 & 0,212 & 0,004 \\
\hline & 3,4 & 0,879 & 0,381 & 0,027 & & 5,4 & 0,889 & 0,213 & 0,004 \\
\hline & 3,6 & 0.956 & 0,386 & 0,025 & & 5,6 & 0,932 & 0,214 & 0,004 \\
\hline & 3,8 & 1,034 & 0.391 & 0,024 & & 5,8 & 0,975 & 0,215 & 0,004 \\
\hline & 4,0 & 1,112 & 0,396 & 0,023 & $(0,5)$ & 6,0 & 1,018 & 0,216 & 0,004 \\
\hline & 4,2 & 1,191 & 0.400 & 0,022 & & 6,5 & 1,126 & 0,218 & 0,004 \\
\hline & 4,4 & 1,271 & 0,405 & 0,022 & & 7,0 & 1,236 & 0,219 & 0.003 \\
\hline & 4,6 & 1,353 & 0,409 & 0,021 & & 7,5 & 1,346 & 0.221 & 0.003 \\
\hline & 4,8 & 1,435 & 0.414 & 0,022 & & 8,0 & 1,457 & 0223 & 0,003 \\
\hline & 5,0 & 1,518 & 0,418 & 0,022 & & 8,5 & 1,569 & 0.224 & 0,003 \\
\hline & 5,2 & 1,602 & 0,422 & 0,022 & & 9,0 & 1,681 & 0,226 & 0,003 \\
\hline & 5,4 & 1,687 & 0,427 & 0,023 & & 9,5 & 1,794 & 0,227 & 0,003 \\
\hline & 5,6 & 1,772 & 0,431 & 0,023 & & 10,0 & 1,908 & 0,228 & 0,003 \\
\hline & 5,8 & 1.859 & 0.436 & 0,024 & & 10,5 & 2,022 & 0,230 & 0,003 \\
\hline & 6,0 & 1,946 & 0.441 & 0,025 & & 11.0 & 2,138 & 0.231 & 0,003 \\
\hline \multirow[t]{12}{*}{$\cdot$} & 6,2 & 2,035 & 0,446 & 0,026 & & 11,5 & 2,254 & 0.233 & 0,003 \\
\hline & 6,4 & 2,124 & 0,451 & 0028 & & 12,0 & 2,371 & 0.235 & 0,004 \\
\hline & 6,6 & 2,215 & 0,457 & 0.029 & & 12,5 & 2,489 & 0.237 & 0,004 \\
\hline & 6,8 & 2,307 & 0.464 & 0.031 & & 13,0 & 2,608 & 0,239 & 0,004 \\
\hline & 7,0 & 2,400 & 0,470 & 0,034 & & 13,5 & 2.728 & 0.241 & 0,005 \\
\hline & 7,2 & 2.494 & 0.477 & 0,036 & & 14,0 & 2,849 & 0,244 & 0,005 \\
\hline & 7,4 & 2,590 & 0,484 & 0,039 & & 14,5 & 2,972 & 0246 & 0,006 \\
\hline & 7,6 & 2,687 & 0,493 & 0,043 & & 15,0 & 3096 & 0,249 & 0,006 \\
\hline & 7,8 & 2,786 & 0,502 & 0,047 & & 15,5 & 3.221 & 0.253 & 0,007 \\
\hline & 8,0 & 2887 & 0,512 & 0,052 & & 16,0 & 3,348 & 0,257 & 0,008 \\
\hline & 8,2 & 2990 & 0,523 & 0.058 & & 16.5 & 3,478 & 0.261 & 0,009 \\
\hline & 8,4 & 3,093 & 0,536 & 0,065 & & 17,0 & 3,610 & 0,267 & 0,011 \\
\hline \multirow[t]{5}{*}{ - } & 8,6 & 3.201 & 0,550 & 0,073 & & & & & \\
\hline & 8,8 & 3.311. & 0,565 & 0,084 & & & & & \\
\hline & 9,0 & 3,425 & 0,583 & 0.096 & & & & & \\
\hline & 9,2 & 3,543 & 0,605 & 0.104 & & & & & \\
\hline & 9,4 & 3,665 & 0,629 & 0,122 & & & & & \\
\hline
\end{tabular}

1) Wir geben im folgenden nicht mehr alle Zwischenwerte. Die eingeklammerten 
Fü r $2-1$-Wertigkeit z 2 .

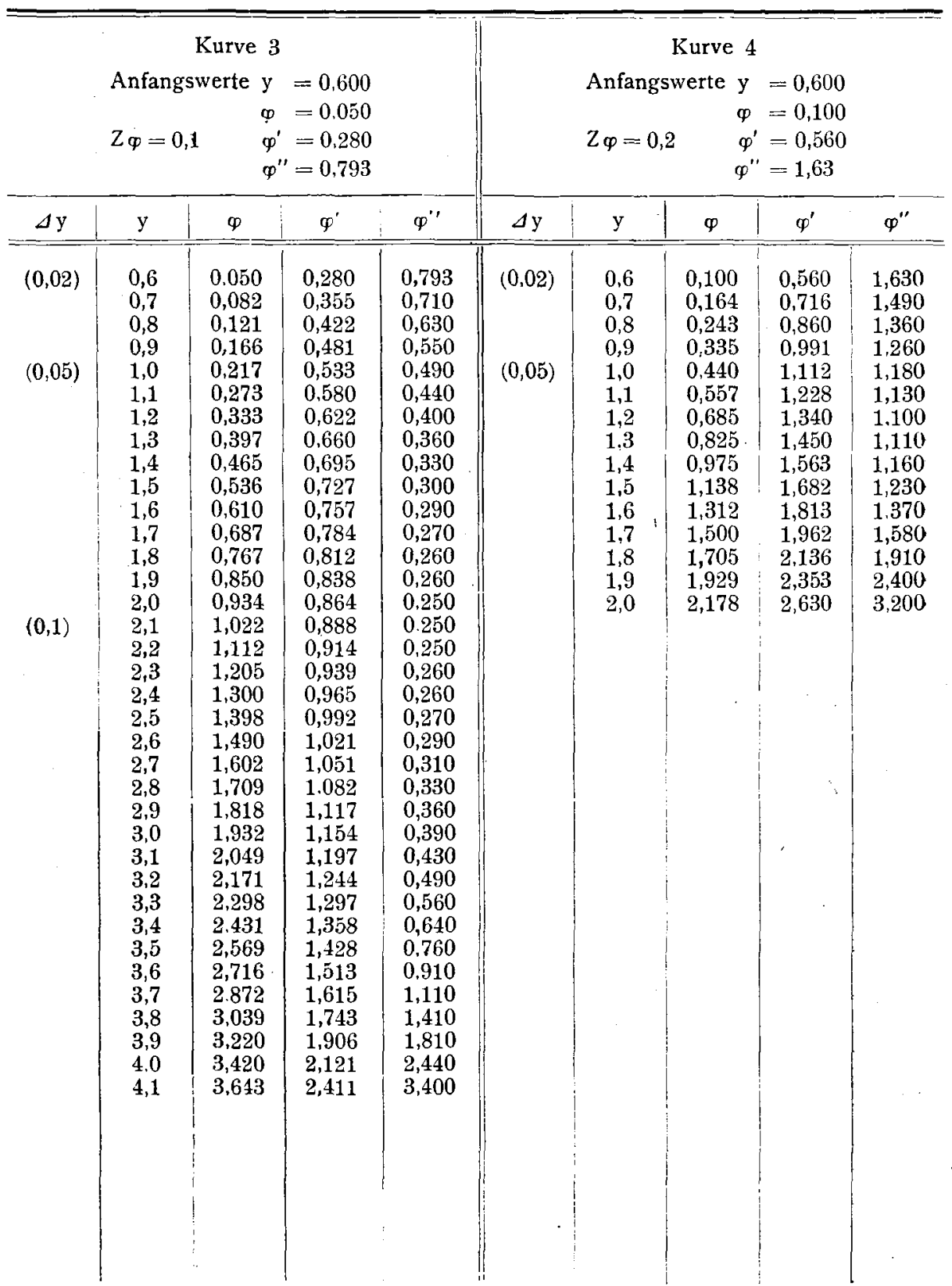

Zahlen geben jeweils die Schrittgröße an. 
Tabelle 3.

Für 3-1-Wertigkeit z 3 .

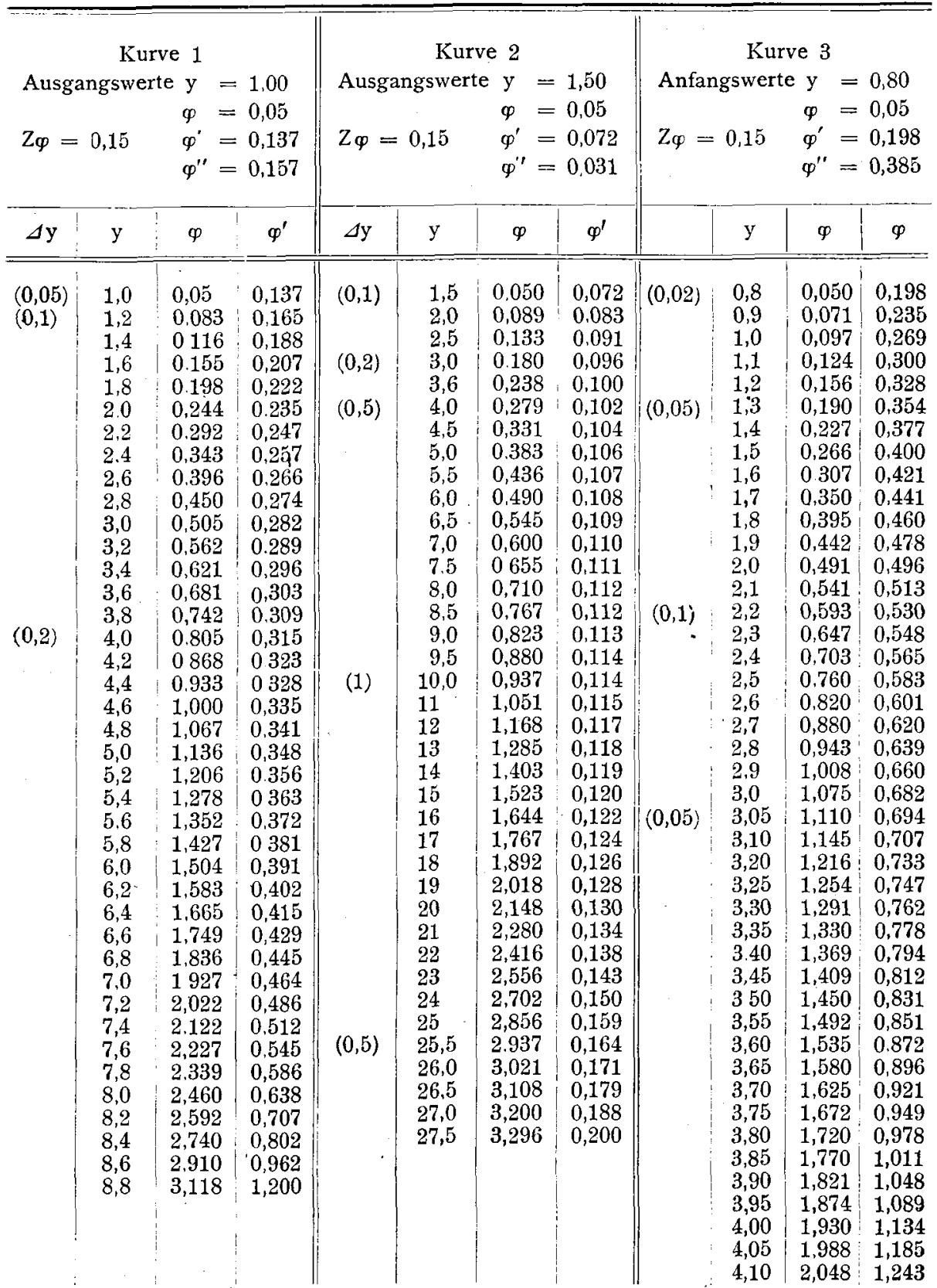


Tabelle 4.

Für 1-4-Wertigkeit $z$ 4.

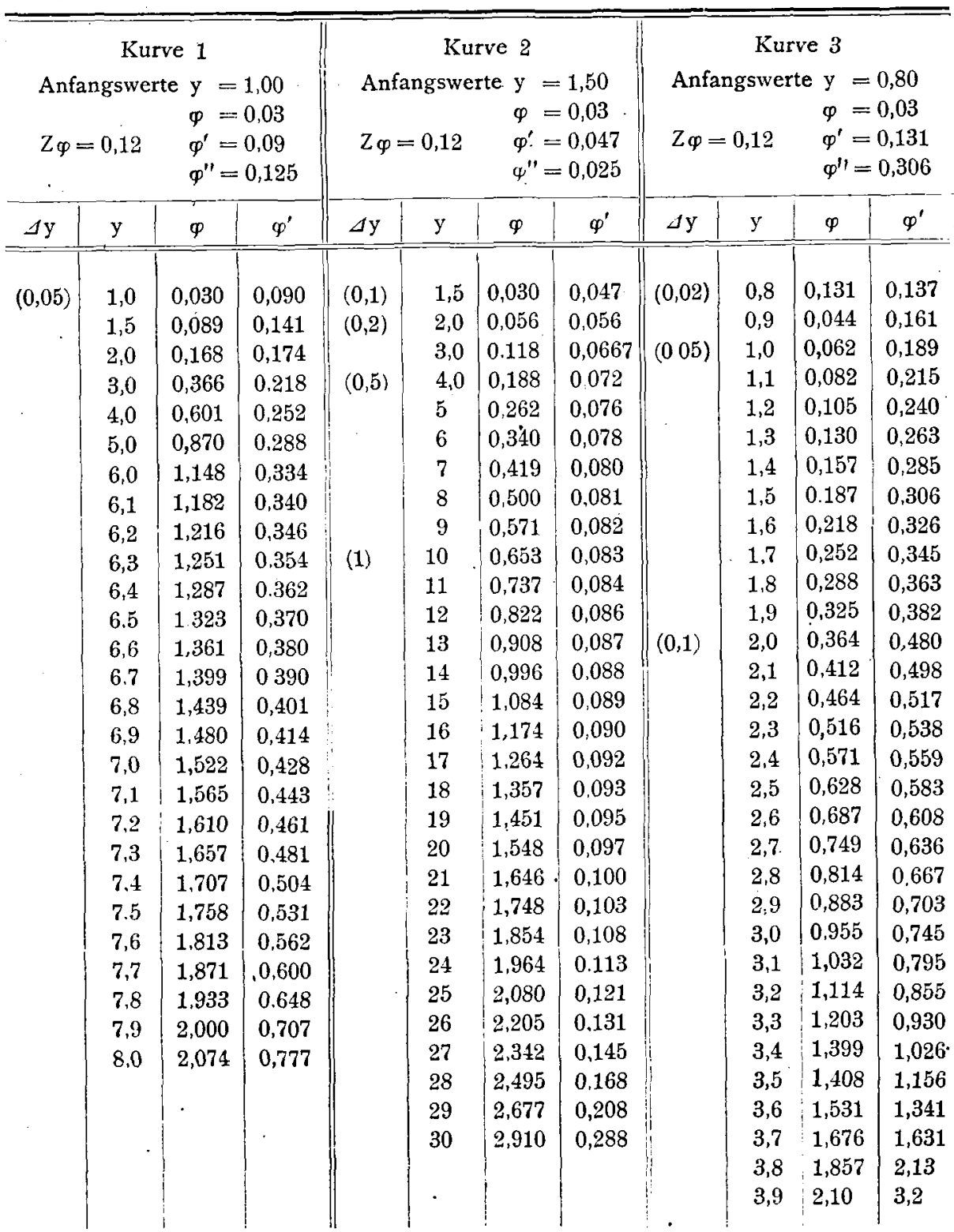


paare $\xi$, c benutzt, sondern haben die gemessenen Punkte durch Kurven interpoliert (Fig. 5) - was leicht möglich ist - und dann zusammengehörende $\xi$, c-Werte auf diesen Interpolationskurven herausgegriffen.

Leider konnte mir Herr Prof. Dr. H. Freundli $\mathrm{ch}^{1}$ ) keine genauere Angaben über die Micellenradien machen. Die Teilchen der betreffenden Sole waren meist amikronisch und dürften bestimmt einen Radius kleiner als $50 \mu \mu$ haben.

Eine Kenntnis dieses Radius ist aber zur Durchführung unserer Rechnung unumgänglich nötig. Wir haben uns deshalb auf folgende Weise geholfen:

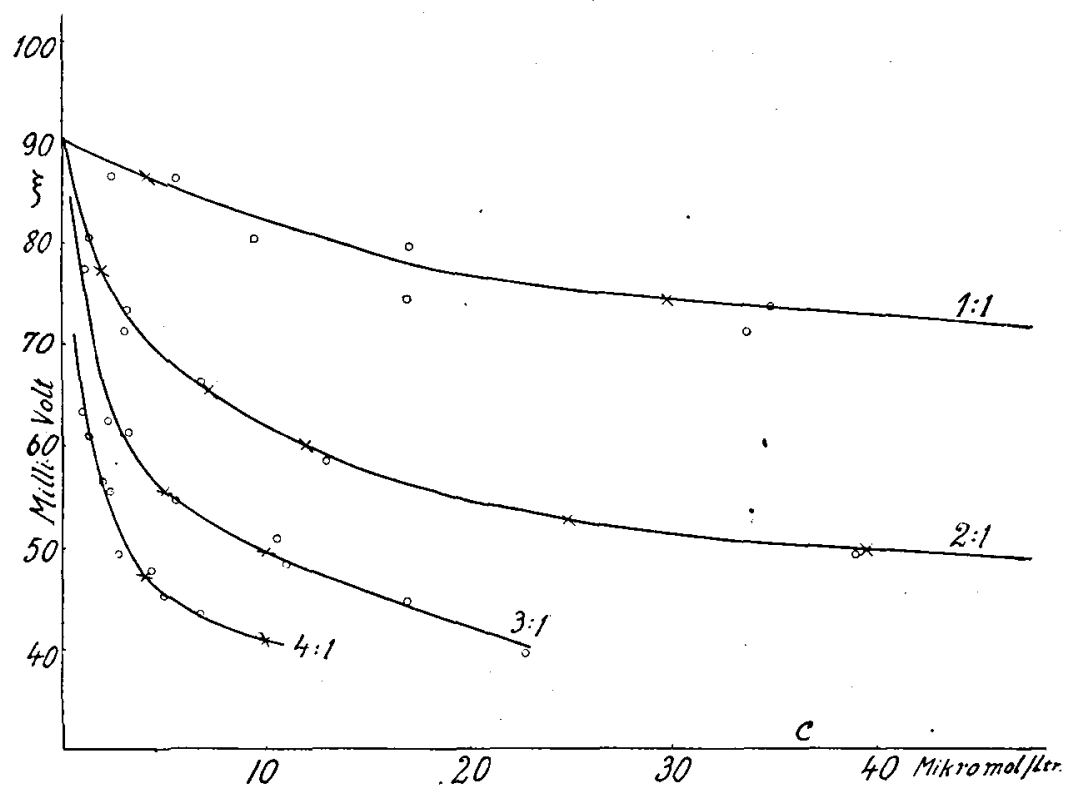

Fig. 5.

Die $\circ$ sind die Messungen von Freundlich und $\mathrm{Zeh}$, die $\times$ sind die Punkte, die zur Berechnung von Fig. 6 benützt wurden.

Wir betrachten ein bestimmtes Wertepaar $c, \xi$ aus den Kurven von Freundlich und $Z$ eh. Wir wählen nun für den Teilchenradius $R$ irgendeinen möglichen Wert (z. B. $20 \mu \mu)$ und bestimmen nun aus den theoretischen Kurven den Wert $\sigma$, den dieses Teilchen haben müßte, $\mathrm{um}$ bei dieser Konzentration $\mathrm{c}$ das gegebene Potential $\xi \mathrm{zu}$ zeigen. Ein anderer Wert R (z. B. $10 \mu \mu)$ liefert für die gleichen c, $\xi$-Werte eine

1) Nach brieflicher Mitteilung von Herrn Prof. H. Freundlich. Ich bin Herrn Prof. Freundlich für das Interesse, das er der Arbeit entgegengebracht hat, sehr zu Dank verpflichtet. 
andere Teilchenladung $\sigma^{\prime}$. Man kann so $z$ u jedem gegebenen Wertepaar c, $\xi$ das entsprechende $\sigma$ als Funktion des Teilchenradius R bestimmen. Wir haben ${ }^{1}$ ) diese Kurven in Fig. 6 für einige verschiedene Konzentrationen verschiedener Elektrolyte dargestellt.

Die einzelnen Punkte dieser $\sigma(R)$-Kurven haben wir tatsächlich folgendermaßen berechnet: Durch die Angabe von c, $\xi$ kennen wir $\varphi_{0}=\frac{\xi}{\mathrm{A}}$. Wir betrachten daher auf einer unserer Kurven, die den Wertigkeiten des betreffenden Elektrolyten entspricht, denjenigen Punkt, für welchen $\varphi=\varphi_{0}$. Aus der Kurve oder der Tabelle lesen wir die zugehörigen Werte von $y_{0}$ und $\varphi_{0}^{\prime}$. Da auch die Konzentrationen bekannt sind, entspricht $\mathrm{y}_{0}$ einem Radius $\mathrm{R}=\frac{\lambda}{\mathrm{y}_{0}}$ und (21b) liefert das zugehörige

$$
\sigma=\frac{\mathrm{DA}}{4 \pi \lambda} \mathrm{y}_{0}^{\prime} \varphi_{0}^{\prime} \text {. }
$$

Wir haben damit die Interpolation zwischen den verschiedenen $\varphi$ (y)-Kurven umgangen und direkt R-Werte gewählt. die Punkte auf den berechneten Kurven ergeben.

In den Kurven der Fig. 6 sind nicht die Werte von $\sigma$ direlst eingetragen, sondern dazu proportionale Werte $\sigma^{*}$

$$
\begin{gathered}
\sigma^{*}=\frac{\sigma}{\beta}=\sigma \frac{4 \pi \mu}{\mathrm{DA}}=\sqrt{\Sigma c_{\mathrm{i}}} \mathrm{y}_{0}^{2} \varphi_{0}^{\prime} \\
\beta=\frac{\mathrm{DA}}{4 \pi \mu} .
\end{gathered}
$$

Der Proportionalitätsfaktor hat den Wert .

$$
\beta=12,48 \text {. }
$$

Diese $\sigma^{*}(R)$-Kurven zeigen nun die entscheidende Tatsache, daB sie sich alle in einem Punkte schneiden

$$
\begin{aligned}
& \mathrm{R}_{0}=15,8 \mu \mu . \\
& \sigma^{*}{ }_{0}=105 .
\end{aligned}
$$

Die Interpolation der einzelnen $\sigma^{*}(\mathrm{R})$-Kurven ist so gut bestimmt, daß der Schnittpunkt mit ziemlicher Genauigkeit für alle Kurven der 1-1- und 1-2wertigen Elektrolyte derselbe ist.

Diese Tatsache dürfte nicht einem Zufall zuzuschreiben sein, sondern sie bedeutet einfach, daß $R_{0}$ der wirkliche Teilchenradius ist und daß bei allen Konzentrationen die Teilchenladung dieselbe ist.

Wäre der Teilchenradius kleiner als $R_{\mathbf{0}}$, so müßten wir eine Entladung, wäre er größer, eine Aufladung des Teilchens mit wachsender

1) Siehe Anhang zum 2. Teil. 
Elektrolytkonzentration annehmen. Der Wert von $R_{0}=15,8 \mu \mu$ ist aber sicherlich von einer Größe, daß er dem benutzten Sol entsprechen

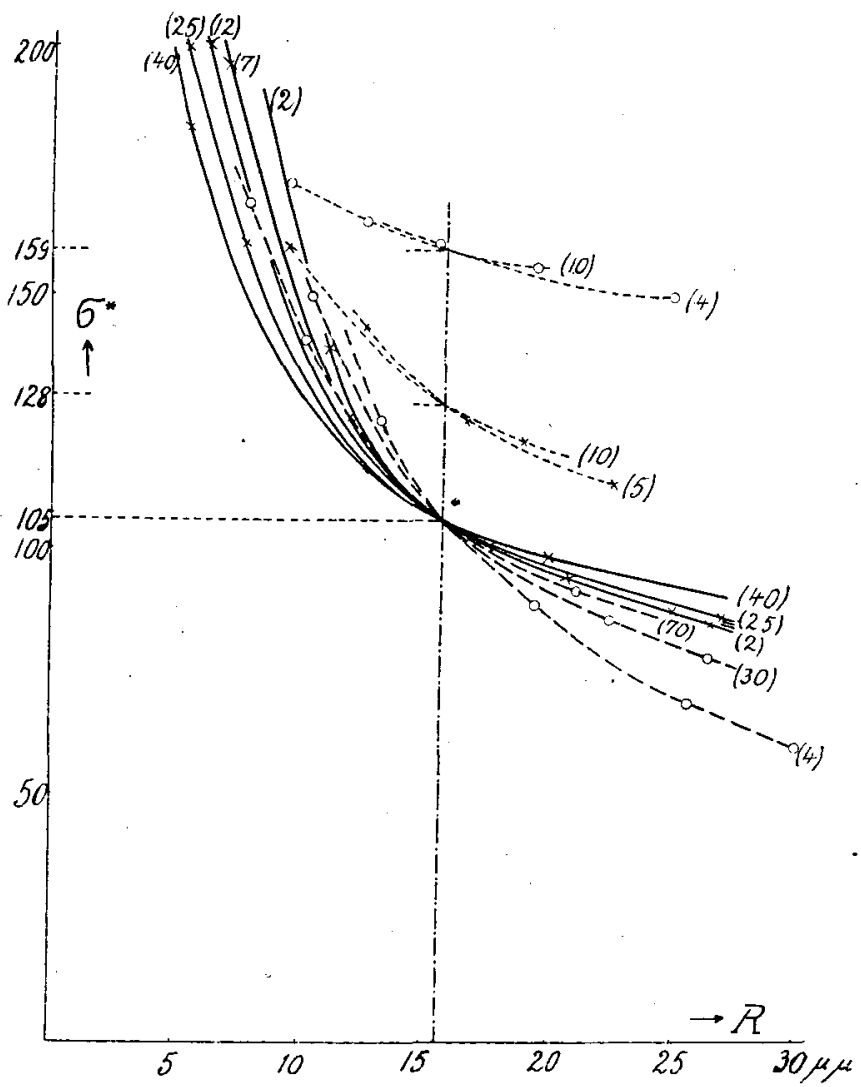

Fig. 6.

- - o- - - für $1: 1$-Wertigkeit für $2: 1$-Wertigkeit

-...- $\times \ldots+\ldots$ für $3: 1$-Wertigkeit -...-. - für 4:1-Wertigkeit

Die eingeklammerten Zahlen geben die Elektrolytkonzentrationen in Mikromol pro Liter. Sie beziehen sich auf die verschiedenen $\sigma^{*}(\mathrm{R})$-Kurven.

dürfte. Auch der Wert der Teilchenladung ergibt sich von zu erwartender Größe. Aus $\sigma^{*}{ }_{0}=105$ wird:

$$
\sigma_{0}=\beta \sigma^{*}{ }_{0}=1310 \text { el. st. E. }
$$

Das bedeutet eine Totalladung der Teilchen von

$$
\mathrm{E}=411 \cdot 10^{-10} \mathrm{el} \text {. st. E. } \sim 86 \text { Elektronen. }
$$

Wir finden somit das Resultat:

Die Kurven von Freundlich und $Z$ eh für die Abhängigkeit des elektrokinetischen Potentials von der Konzentration 1-1- und 1-2. 
wertiger Elektrolyte können ohne Adsorption erklärt werden, wenn man den Teilchen einen Radius $\mathrm{R}=15,8 \mu \mu$ und eine konstante Ladung von 86 Elektronen zuschreibt.

Für die 1-3- und 1-4wertigen. Elektrolyte ist der Schnittpunkt der $\sigma(R)$-Kurven nicht so gut bestimmt. Er erscheint in beiden Fällen auch für den gleichen Radius $R_{0}=15,8 \mu \mu$ zu existieren, aber für eine größere Teilchenladung $\sigma_{0}^{*}=128$ bzw. $\sigma_{0}^{*}=159$. Wir vermuten, daß diese Abweichungen nur durch Ungenauigkeit der möglichen Konzentrationsangaben hervorgerufen wird, da bei diesen Elektrolyten der Potentialabfall für Konzentrationen kleiner als ein Mikromol auftritt, wo die Fehlerquellen sehr großen Einfluß haben.

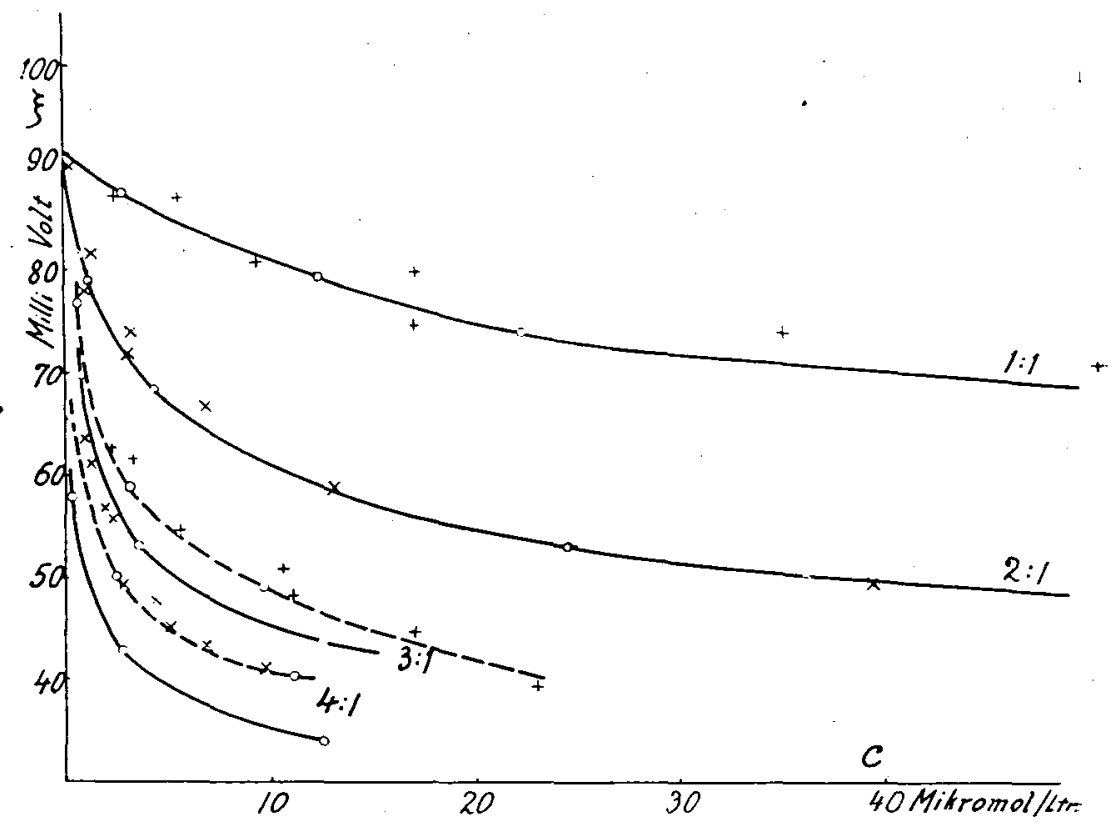

Fig. 7.

- berechnete Punkte

$x,+$ gemessene Punkte.

In Fig. 7 haben ${ }^{1}$ ) wir mit dem konstanten Wert $\mathrm{R}_{0}$ die $\sigma_{0^{-}}$ Kurven für alle vier Elektrolyte berechnet und die gemessenen Werte als Punkte eingetragen. Für die 1-3- und 1-4wertigen Elektrolyte sind daneben die Kurven für $\sigma_{0}^{*}=128$ bzw. $\sigma_{0}^{*}=159$ punktiert angedeutet. Die Übereinstimmung dürfte als sehr gut bezeichnet werden.

Es zeigt sich in Fig. 7 sehr schön unsere Behauptung, daß die Theorieder diffusen Doppelschicht allein ohne jede Adsorption den Abfall der

1) Siehe Anhang zum 2. Teil. 
$\xi$ (c)-Kurven nicht nur qualitativ, sondern auch quantitativ richtig darstellen kann. Ebenso wird der Einfluß der Wertigkeit der Ionen recht gut dargestellt.

Da alle Versuche von Freundlich und $Z \mathrm{eh}$ an dem gleichen $\mathrm{As}_{2} \mathrm{~S}_{3}$ Sol durchgeführt wurden, sollte man in allen Fällen nicht nur den gleichen Radius $\mathrm{R}_{0}$, sondern auch die gleiche Teilchenladung $\sigma_{0}$ erwarten. Tatsächlich liefern aber die Kurven für das konstante $\sigma_{0}^{*}=105 \mathrm{im}$ Falle der 3-1- und 4-1wertigen Elektrolyte einen zu steilen Abfall $\operatorname{der} \xi$ (c)-Kurve gegenüber den gemessenen Kurven.

Die Annahme eines größeren Wertes der Teilchenladung korrigiert zwar diese Differenz, aber wir glauben nicht, daß wirklich eine höhere Aufladung der Teilchen existiert. Wir glauben vielmehr, daß bei den höherwertigen Ionen bereits ein Einfluß der Ionengröße eine Rolle spielt. Bei den geringen Potentialen, die diese Ionen hervorrufen, ist die Dicke der Doppelschicht klein. Infolge der endlichen Ionengröße (es handelt sich hier um große Komplexionen) können aber die Ionen sich nicht beliebig der Teilchenoberfläche nähern, und die Doppelschicht ist dicker, als sie nach unserer Theorie sein sollte. Somit ergibt sich auch ein etwas größeres Potential. Bei den 2-1- und 1-1wertigen Elektrolyten spielt dieser Einfluß der Ionengröße noch keine so große Rolle, da dort das Potential größer ist und sich die Ionenatmosphäre bedeutend weiter in die Flüssigkeit erstreckt.

Damit dürfte die im Abschnitt I aufgestellte Behauptung, daß die Änderung der diffusen Doppelschicht in den diskutierten Fällen der wesentliche Effekt zum Verständnis der $\xi$ (c)-Kurven ist, begründet sein.

IV.

Es dürfte an Hand der hier entwickelten Darstellung möglich sein, in anderen Fällen, wo die $\boldsymbol{\xi}$ (c)-Kurve nicht diesen einfachen Verlauf zeigt, die Adsorptionsänderung näher zu ergründen. Durch Berücksichtigung der kapillarelektrischen Erscheinungen sollte es damit mög. lich sein, auch die Konstitution der inneren Ionenschichten experimentell zu erforschen. Für den ersten Zweck ist nur nötig, daß man neben der $\xi$ (c)-Kurven auch den Teilchenradius mit einiger Genauigkeit kennt.

Eine genauere Berücksichtigung der Ionengröße und der Hydratation dürfte heute noch verfrüht sein; sie dürfte im wesentlichen darauf hinauskommen, daß man an Stelle der Konzentrationen die bekannten „Aktivitäten“ einführt. Qualitativ hat bereits Wiegner ${ }^{1}$ ) zeigen

1) Wiegner, Koll.Zeitschr. 36, 341 (1925). 
können, daß der Hydratationseinfluß auf Grund der Theorie der diffusen Doppelschicht verstanden werden kann.

Wenn unsere hier entwickelten Anschauungen zutreffen, so wird daraus auch der Einfluß des "nicht entladenden" Ions verständlich. Das gleich geladene Ion in der Doppelschicht vergrößert die Dicke derselben. Wenn daher die Wertigkeit dieser Ionen vergrößert wird und entsprechend ihre Anzahl verkleinert, so befinden sich infolge der größeren abstoßenden Kräfte und der kleineren Anzahl weniger solcher Ionen innerhalb der Doppelschicht. Ihr Einfluß ist dann trotz der größeren Wertig. keit kleiner. Bei einer höheren Wertigkeit des nicht entladenden Ions ist daher die Dicke der Doppel. schicht kleiner, das Potential somit auch kleiner. Es wird daher der Abfall der $\xi(c)$. Kurve für ein höherwertiges gleich geladenes Ion steiler, aber

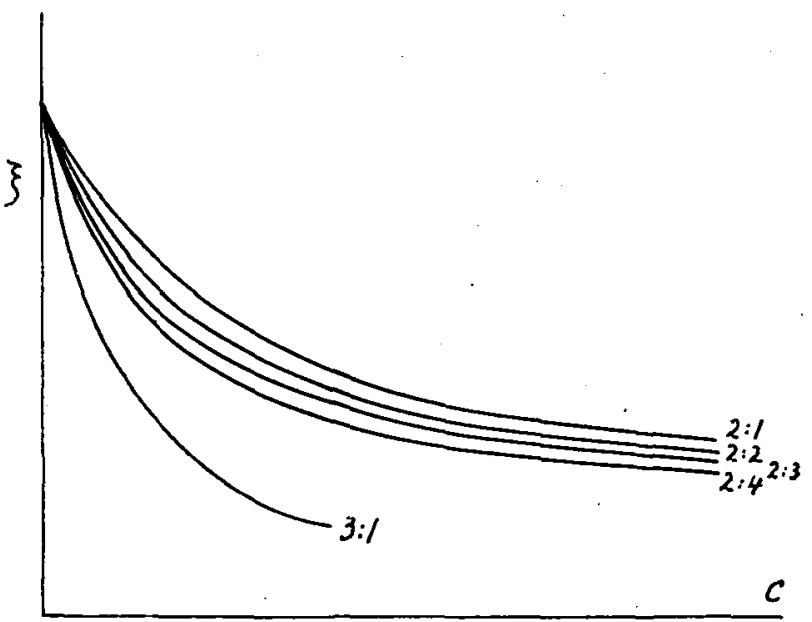

Fig. 8. der Einfluß ist bedeutend kleiner als der Einfluß der Wertigkeit des entladenden Ions. Wir haben in Fig. 8 diesen Einfluß an Hand der D e b y e schen Näherung skizziert für einen Elektrolyten $\mathrm{A}^{2}, \mathrm{~B}_{2 / \mathrm{z}}^{-}$, wo dass entladende Ion $z$ weiwertig ist, während wir dem Ion B die Valenz 1, 2, 3 erteilt haben.

Natürlich gibt die Theorie auch den Einfluß der Dielektrizitätskonstanten qualitativ so, wie er beobachtet wird. Auch eine Einwirkung der Temperatur kann auf Grund derselben diskutiert werden: dies könnte möglicherweise ein wichtiger Punkt zur Prüfung der Theorie sein.

Einen ganz bemerkenswerten Einfluß spielt in unserer Betrachtung die Größe der Micellen. Für den Verlauf der $\xi(c)$-Kurven spielt er zwar nur eine merkliche Rolle, wenn man zwei Teilchensorten vergleicht, von denen die eine einen sehr kleinen $(1-5 \mu \mu)$, die andere einen großen (über $50 \mu \mu$ ) Radius hat. Experimentell wurde dieser Einfluß noch nicht genauer untersucht.1) Er wäre in folgender Richtung

1) Siehe Anmerkung S. 30. 
zu erwarten: Nehmen wir an, die verschieden großen Micellen hätten die gleiche 'spezifische Ladung $\sigma$, so wäre das Potential $\xi$ des kleineren Teilchens stets kleiner als dasjenige des größeren. Dagegen würde sich das

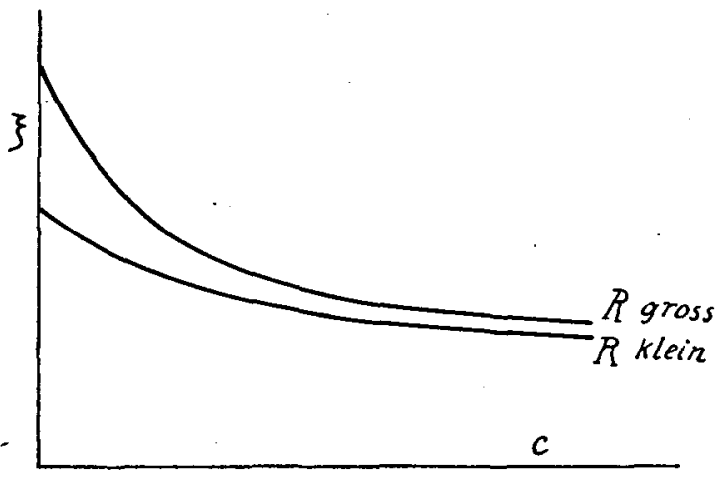

Fig. 9.

kleine Potential langsamer ändern mit der Erhöhung der Elektrolytkonzentration (siehe Fig. 9).

In Wirklichkeitscheint die Annahme gleicher spezifischer Ladung nicht zuzutreffen. Hevesey') hat aus experimentellen Daten geschlossen, daß es im Gegenteil scheint, daß sich die kleinen Teilchen relativ stärker aufladen als die großen, so daß - bei verschwindend kleiner Elektrolytkonżentration das Potential $\xi$ das gleiche wird. Nehmen wir daher an, daß für $\mathrm{c}=0$ das Potential $\xi$ für beide Teilchen den gleichen Wert hat, so äußert sich die verschiedene Teilchengröße dadurch, 'daß die $\xi$ (c)-Kurve des kleinen Teilchens langsamer abfällt.

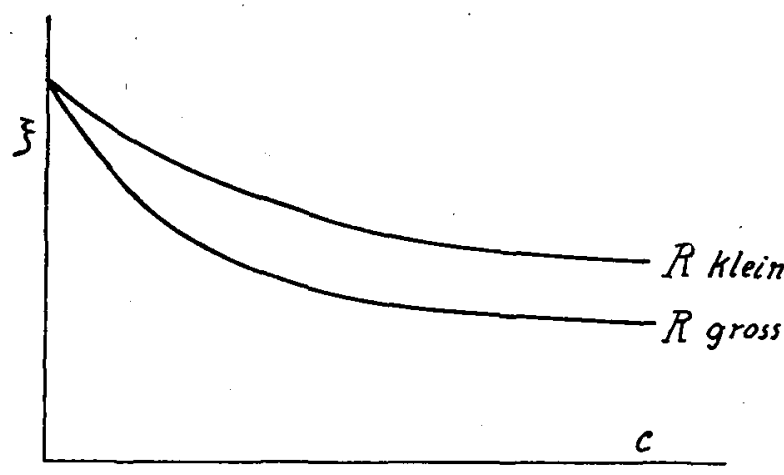

Fig. 10.

Eine große Reihe. von Versuchen sprechen dafür, di $\beta$ das elektrokinetische $\mathrm{Po}_{0}$ tential $\xi$ (c) direkt ein $\mathrm{Maß}$ ist für die Stabilität eines Kolloides, d. h. daß die Koagulationsgeschwindigkeit der làngsamen Flockung eines Sols um so kleiner ist, je größer das Potential $\xi$ ist. Wir müßten somit nach Fig. 10 er warten, daß ein feindisperses Sol stabiler ist als ein grobdisperses bei gleicher Elektrolytkonzentration. Dieser Effekt ist tatsächlich schon konstatiert worden. ${ }^{2}$ )

1) Hevesey, Koll.-Zeitschr. 21, 129 (1917).

2) Nach Mitteilung von Herrn Prof. G. Wi egner haben sowohl Dr. Galay als auch Dr. Tuotila folgende Tatsache konstatiert: Leitet man in einem Gemisch von grobdispersem und amikronischem Sol (Gold, Ton) die langsame Koagulation ein, so flockt das grobe Sol bald aus und bildet einen Bodensatz. Die überstehende Fliissigkeit enthält aber noch sehr viel Amikronen, die erst bei Vergrößerung der Elektrolytkonzentration ausflocken. 
Wir möchten zum Schlusse noch auf einige kurze Bemerkungen über die langsame Koagulation und, die im ersten Teil aufgeworfene Frage des autokatalytischen Verlaufes derselben zurückkommen. Nach der Annahme von v. S moluchowskil) sollte die langsame Koagulation gleich verlaufen wie die rasche, mit dem einzigen Unterschied, daß die Koagulationszeit $\mathrm{T}=\frac{1}{\mathrm{NW}}$ eine größere ist, weil die Wahrscheinlichkeit $\mathrm{W}$ eines Zusammenstoßes mit einem Faktor $\lambda<1$, der von der Elektrolytkonzentration abhängt, multipliziert wèrden muß :

$$
\mathrm{T}=\frac{1}{\mathrm{~N} \cdot \lambda \mathrm{W}} .
$$

Nach unserer Theorie muß der Faktor $\lambda$ auch noch von der Größe des Teilchenradius abhängen, und zwar so, daß $\lambda$ mit dem Teilchenradius größer wird. $\mathrm{Da}$ aber während der Koagulation der mittlere Teilchenradius anwächst, so ist zu erwarten, daß im Verlaufe der langsamen Koagulation die Koagulationszeit kleiner wird, d.h., da $B$ sich die Flockung beschleunigt. Wir haben damit eine neue Ursache gefunden, die zusammen mit der im ersten Teil besprochenen Keimwirkung der großen Teilchen für den autokatalytischen Verlauf der langsamen Koagulation spricht. Wir sehen nun auch ein, daß der die Keimwirkung charakterisierende Faktor $\gamma$ für die langsame Flockung eine viel kompliziertere Funktion der beiden Teilchenradien sein kann als in der Theorie der raschen Koagulation.

Der autokatalytische Verlauf der langsamen Flockung ist noch nicht direkt durch ultramikroskopische Auszählungen nachgewiesen worden. Er äußert sich nur in der Änderung vieler Kolloideigenschaften im Verlaufe der Flockung. v.Smoluchowski1) hat bekanntlich den Effekt als einen nur scheinbaren bezeichnet, indem er die aus Viskositätsmessungen gefundenen Kurven durch Änderung des totalen Micellvolumens erklärte. Da der Effekt aber auch bei anderen Kolloideigenschaften deutlich zum Ausdruck kommt, so dürfte es nach Freundlich ${ }^{2}$ ) „nicht endgültig ausgemacht sein", daß er nicht existiert.

1) v. Smoluchowski, Zeitschr. f. phys. Chem. 92, 129 (1917).

2) H. Freundlich, Kapillarchemie (2. Aufl.), 604. 


\section{Anhang zum zweiten Teil. \\ Berechnung der Kurven $\boldsymbol{\xi}$ (c) (Fig. 7).}

Um zur Berechnung der Kurven von Fig. 7 die Interpolation zwischen den $\varphi(\mathrm{y})$-Kurven zu umgehen, geht man folgendermaßen vor:

Wir suchen auf den berechneten $\varphi(\mathrm{y})$-Kurven diejenigen Punkte, die den gegebenen Werten von $R_{0}$ und $\sigma_{0}$ (bzw. $\sigma_{0}^{*}$ ) entsprechen. Nun ist nach (27)

und außerdem

$$
\begin{gathered}
\sigma^{*}=\mathrm{y}^{2} \frac{\mathrm{d} \varphi}{\mathrm{dy}} \sqrt{\Sigma \mathrm{c}_{\mathrm{i}}} \\
\mathrm{R}=\frac{\lambda}{\mathrm{y}}=\frac{\mu}{\mathrm{y} \sqrt{\Sigma \mathrm{c}_{\mathrm{i}}}} .
\end{gathered}
$$

Durch Multiplikation dieser beiden Gleichungen erhält man

$$
\frac{\sigma^{*} \mathrm{R}}{\mu}=\mathrm{M}=\mathrm{y} \frac{\mathrm{d} \varphi}{\mathrm{d} \mathbf{y}} \text {. }
$$

Die Größe $M=\frac{\sigma^{*} R}{\mu}$ ist eine Konstante, in unserem Falle

$$
\mathrm{M}=\frac{105 \cdot 15,8 \cdot 10^{-7}}{4,32 \cdot 10^{-5}}=3,84 .
$$

Für die 3-1- bzw. 4-1wertigen Elektrolyte hat man $M=4,68$ bzw. 5,82 .

Gleichung (31) muß durch die gesuchten Punkte erfüllt sein, und bildet daher eine Bedingung, die auf jeder $\varphi(\mathrm{y})$-Kurve einen Punkt eindeutig bestimmt (wir haben sie in den Kurven markiert). Die Tabellen geben sofort den Wert des Potentials $\varphi$, während die ent. sprechende Konzentration aus y gefunden wird mit Hilfe von (30):

$$
\Sigma c_{i}=\left(\frac{\mu}{R}\right)^{2} \frac{1}{y^{2}}=\frac{N}{y^{2}} .
$$

Der Faktor $N=\left(\frac{\mu}{R}\right)^{2}$ ist wiederum eine Konstante, die sich in unserem Fall ergibt zu

$$
\mathrm{N}=\left(\frac{4,32 \cdot 10^{-5}}{15,8 \cdot 10^{-7}}\right)^{2}=746,5
$$

Für die Elektrolyte vom Typ $A_{1}^{(+2)} B_{2}$ hat man noch $\Sigma c_{i}=c(z+1)$, so daß man erhält:

$$
c=\frac{N}{(z+1)} \frac{1}{y^{2}} .
$$


Durch dieses Verfahren erhält man aus den verschiedenen Kurven die folgenden zusammengehörigen Werte von $\xi$ und $c$ :

$1-1 \cdot$ Wertigkeit.

\begin{tabular}{|c|c|c|c|c|c|}
\hline \multirow{2}{*}{ Kurve } & \multicolumn{3}{|c|}{ nach $(31)$} & \multicolumn{2}{|c|}{ nach (33) } \\
\hline & $\mathrm{y}$ & $\varphi^{\prime}$ & $\varphi$ & $\xi=A \varphi$ & c \\
\hline $\begin{array}{l}1 \\
2 \\
3 \\
4 \\
5\end{array}$ & $\begin{array}{c}5,5 \\
11,5 \\
4,1 \\
2,18 \\
1,79\end{array}$ & $\begin{array}{l}0,730 \\
0,335 \\
0,936 \\
1,760 \\
2,142\end{array}$ & $\begin{array}{l}3,18 \\
3,491 \\
2,971 \\
2,50 \\
2,33\end{array}$ & $\begin{array}{l}79,5 \\
87,3 \\
74,3 \\
62,5 \\
58\end{array}$ & $\begin{array}{c}12,35 \\
2,85 \\
22,2 \\
78,5 \\
116\end{array}$ \\
\hline \multicolumn{6}{|c|}{ 2-1-Wertigkeit. } \\
\hline $\begin{array}{l}1 \\
2 \\
3 \\
4\end{array}$ & $\begin{array}{c}7,7 \\
15,3 \\
3,17 \\
1,80\end{array}$ & $\begin{array}{l}0,497 \\
0,25 \\
1,21 \\
2,13\end{array}$ & $\begin{array}{l}2,736 \\
3,17 \\
2,13 \\
1,70\end{array}$ & $\begin{array}{l}68,4 \\
79,2 \\
53,2 \\
42,5\end{array}$ & $\begin{array}{l}4,2 \\
1,06 \\
24,8 \\
77\end{array}$ \\
\hline \multicolumn{6}{|c|}{ 3-1-Wertigkeit. } \\
\hline 3 & $\begin{array}{c}7,4 \\
(7,85 \\
24,6 \\
(26,4 \\
\mathbf{3 , 8 4} \\
(4,02\end{array}$ & $\begin{array}{l}0,52 \\
0,595 \\
0,156 \\
0,123 \\
1,0 \\
1,16\end{array}$ & $\begin{array}{l}2,14 \\
2,37 \\
2,79 \\
3,08 \\
1,76 \\
1,95\end{array}$ & $\begin{array}{l}53,5 \\
59,3 \\
70 \\
77 \\
44 \\
48,7\end{array}$ & $\begin{array}{c}3,4 \\
3,0) \\
0,31 \\
0,27) \\
12,6 \\
9,55)\end{array}$ \\
\hline \multicolumn{6}{|c|}{$4-1$-Wertigkeit. } \\
\hline 3 & $\begin{array}{c}7,45 \\
(7,95 \\
26,9 \\
(28,8 \\
63,47 \\
(3,68\end{array}$ & $\begin{array}{l}0516 \\
0,73 \\
0,145 \\
0,200 . \\
1,11 \\
1,59\end{array}$ & $\begin{array}{l}1,73 \\
2,03 \\
2,33 \\
2,64 \\
1,37 \\
1,64\end{array}$ & $\begin{array}{l}43,3 \\
50,2 \\
58,2 \\
66 \\
34,2 \\
41,0\end{array}$ & $\begin{array}{c}2,71 \\
2,36) \\
0,20 \\
0,18) \\
12,4 \\
11,0)\end{array}$ \\
\hline
\end{tabular}

Die eingeklammerten Zahlen unter 3-1- und 4-1-Wertigkeit beziehen sich auf die Teilchenladung $\sigma_{0}^{*}=128 \mathrm{bzw} .159$. 


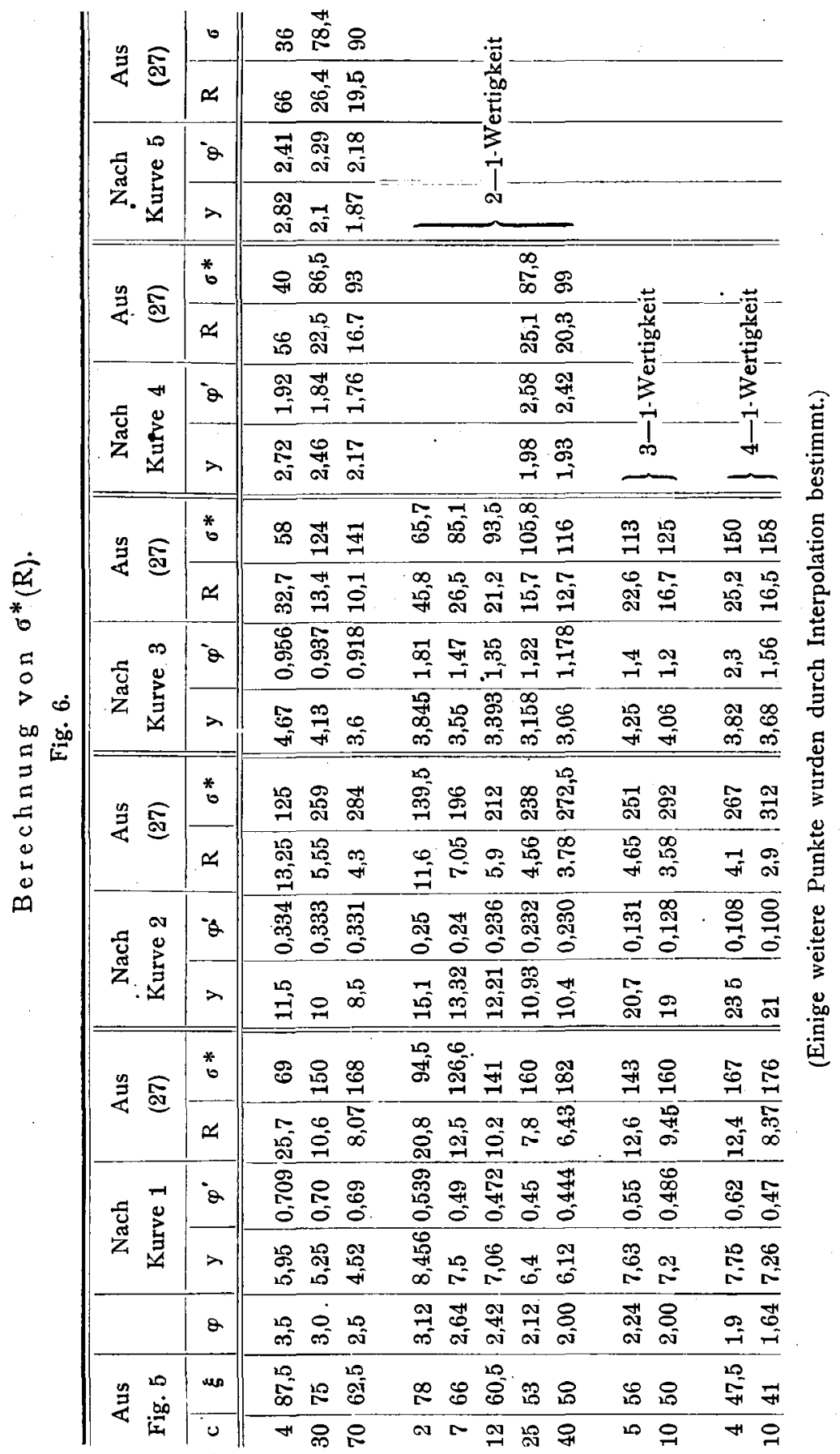




\section{Lebenslauf.}

Ich wurde geboren am 27. Oktober 1900 in Amriswil (Thurgau) als Sohn des Ernst Müller, Landwirt, von und in Amriswil und der Mathilde (sel.) geb. Meier. Meine erste Schulbildung erhielt ich an den Primar- und Sekundarschulen von Amriswil. Im. Frühjahr 1916 trat ich als Schüler in die technische Abteilung der Thurgauischen Kantonsschule in Frauenfeld, wo ich im Herbst 1919 das Reifezeugnis erhielt. Nach ach $\dot{t}$ Semestern Studium an der Eidgenössischen Technischen Hochschule in Zürich erwarb ich im Sommer 1923 das Diplom als Fachlehrer in Mathematik und Physik. Darauf war ich während zwei Jahren Assistent der Herren Professoren Debye und Scherrer am physikalischen Institut dieser Hochschule, bis ich im Herbst 1925 die Stelle eines Research associate am Massachusetts Institute of Technology in Cambridge (U. S. A.) mit gleichzeitiger Habilitation zum Member of the Staff erhielt. 